\title{
Superlinear Deterministic Top-Down Tree Transducers
}

\author{
Ph.D. Thesis
}

by

Gábor Dányi

Szeged, Hungary 


\section{Contents}

$\begin{array}{ll}\text { Introduction } & 3\end{array}$

1 Preliminaries 9

1.1 Sets and relations . . . . . . . . . . . . . . . . 9

1.2 Strings and string rewriting systems . . . . . . . . . . . . 11

1.3 Trees, tree languages, and tree transformations . . . . . . . . . 12

1.4 Top-down tree transducers . . . . . . . . . . . . . . . . . . 14

1.4.1 Restricted types . . . . . . . . . . . . . 16

1.4.2 Compositions and decompositions . . . . . . . . . . 17

1.4.3 Top-down tree recognizers and recognizable tree languages 20

1.4.4 Minimal deterministic top-down tree recognizers . . . . . . 20

1.4.5 Domain and range tree languages . . . . . . . . . . . 24

2 Properties of sl-dt tree transducers $\quad 27$

2.1 Basic properties . . . . . . . . . . . . . . . . 27

2.2 Domain tree languages . . . . . . . . . . . . . . . . . . . . . . . . . . . . . . . . . . . . . . .

2.3 Range tree languages . . . . . . . . . . . . . . . . . . . . . 41

$3 \quad$ Hierarchy theorems of sl-dt tree transformations $\quad 43$

3.1 The hierarchies $\operatorname{dom}\left(s l-D T^{n}\right)$ and $s l-D T^{n} \ldots \ldots . . . . . .43$

3.2 The hierarchy $t-s l-D T^{n} \ldots \ldots \ldots \ldots$. . . . . . . . 50

4 Compositions with sl-dt tree transformations $\quad 59$

4.1 The problem and the outline of the solution . . . . . . . . . 59

4.2 The decidability of inclusions in the composition monoid . . . . . 64

4.3 The inclusion diagram of normal forms . . . . . . . . . . . . . 72

$\begin{array}{lr}\text { Conclusions } & 88\end{array}$

Összefoglalás (Summary in Hungarian) 91

$\begin{array}{lr}\text { Bibliography } & 96\end{array}$ 


\section{List of Figures}

1.1 Representation of trees . . . . . . . . . . . . . . . 13

3.1 Example trees for hierarchy theorems . . . . . . . . . . . 45

3.2 The hierarchy of $s l-D T^{n} \ldots \ldots \ldots \ldots$. . . . . . . . . . . . . . . . . . . . 51

3.3 The hierarchy of $t-s l-D T^{n} \ldots \ldots \ldots . \ldots . \ldots 58$

4.1 Rewriting rules of $R \ldots \ldots \ldots \ldots$. . . . . . . . . . . . 63

4.2 The inclusion diagram of normal forms . . . . . . . . . . . . 67

4.3 Table of concatenations with the elements in NF (part 1) . . . . 70

4.4 Table of concatenations with the elements in NF (part 2) . . . 71

4.5 Example trees for Lemma 4.3.5 . . . . . . . . . . . . . . . . 79

4.6 The inclusion diagram of suprema . . . . . . . . . . . . . . . . . 82

4.7 The inclusion diagram of suprema and top elements . . . . . . . . 84 


\section{Introduction}

In theoretical computer science tree transducers have been studied since the early seventies. They are finite devices processing terms over ranked alphabets. Such terms are called trees in this area. A tree transducer induces a binary relation over tree sets, called a tree transformation.

Several types of tree transducers have been defined. Namely, the concept of the top-down tree transducer was introduced in [Rou] and [Tha1]. Then the notion of the bottom-up tree transducer was defined in [Tha2]. Later on, in order to increase the transformational capacity, more powerful devices were introduced, such as top-down tree transducers with regular look-ahead (see [Eng2]), macro tree transducers (see [EngVog1]), attributed tree transducers (see [Fül1]), high level tree transducers (see [EngVog2]), modular tree transducers (see [EngVog3]), and high level modular tree transducers (see [Vog]).

In this thesis we shall consider only deterministic top-down tree transducers and tree transformations induced by such tree transducers.

The motivation of studying top-down tree transducers is that they serve as formal models of syntax-directed compilers, thus tree transformations induced by top-down tree transducers are abstract models of translations realized by syntaxdirected compilers, see [Eng4].

Several restricted subtypes of deterministic top-down tree transducers have been defined and investigated. In this thesis we work with, among others, total, linear, nondeleting, and homomorphism deterministic top-down tree transducers.

In our sense, a tree transformation class is generally a class of tree transformations induced by tree transducers of a certain type. Thus we can distinguish the class of deterministic top-down tree transformations, denoted by $D T$, and its subclasses of total, linear, nondeleting, and homomorphism deterministic topdown tree transformations, denoted by $t-D T, l-D T$, nd-DT, and HOM, respectively. Moreover, type properties can be combined resulting more special devices. For instance, we can speak about linear and nondeleting deterministic top-down tree transducers, of which the induced tree transformation class is denoted by $l-n d-D T$.

Investigating a certain type of top-down tree transducers, the questions naturally arise, what sets of trees can be processed by top-down tree transducers of that type, and what sets of trees can occur as results of such processings. For a 
top-down tree transducer, the sets of possible input and output trees are called the domain and the range of the induced tree transformation, respectively.

Tree sets are also called tree languages. Similarly to string languages, for tree languages there also exist finite state recognizers. Using these devices, we can define the classes of recognizable and deterministic recognizable tree languages, see [GécSte4]. It turned out that the domains of deterministic top-down tree transformations are exactly the deterministic recognizable tree languages. Moreover, the class of ranges of linear deterministic top-down tree transformations is exactly the class of recognizable tree languages.

Since tree transformations are binary relations over tree sets, the concept of their composition, denoted by $\circ$, is clear. Moreover, the composition operation can naturally be extended to classes of tree transformations.

Compositions and decompositions of deterministic top-down tree transformation classes are of special interest, because they model consecutive applications of deterministic top-down tree transducers of certain types to tree languages in such a way that the output of a device is the input of its successor. The motivation of studying compositions comes from the fact that applying deterministic top-down tree transducers in succession can yield extra computational power in the sense that the resulting tree transformation cannot be induced in general by a single deterministic top-down tree transducer. Similarly, the investigation of decompositions is motivated by the intention that one would like to know whether a deterministic top-down tree transformation of a certain type could also be induced by the consecutive application of two or more deterministic top-down tree transducers of simpler types.

Top-down tree transducers and top-down tree transformations were studied in a large number of papers.

In pioneer works [Rou], [Tha1], [Eng1], [Eng3], [Bak1], [Bak2] and [Bak3] several restricted types (total, linear, nondeleting, etc.) were defined, the transformational power of different types were compared to each other, and some closure properties of the corresponding tree transformation classes were explored. A good survey of these results can be found in [GécSte4]. Moreover, [FülVág1] also contains important observations concerning closure properties of the class of deterministic top-down tree transformations and its subclasses.

Recognizability of domains and ranges of top-down tree transformations also have been studied very intensively, see [Rou], [Eng2], [GécSte4], [FülVág1], and [FülVág3].

The undecidability of equivalence problem of top-down tree transducers in the general case immediately follows from the result of [Gri] on the undecidability of equivalence problem of GSM's. On the other hand, it turned out that the equivalence is decidable in the deterministic case, see [Ésil] and [Zac]. Moreover, the equivalence problem were studied for some other restricted nondeterministic types in [AndBos]. The decidability of some other properties (injectivity, recognizability of the range tree set, etc.) have also been investigated, see [Ési1], 
[Ési2], [Fül4], and [FülGye].

Compositions and decompositions of tree transformation classes have been studied very intensively. Almost all papers regarding tree transducers contains such results and hence a huge number of decomposition and composition equations have been obtained. It was desirable to find a uniform way for researching this area. In [FülVág4] and [FülVág6] a general method was proposed for developing an algorithm, which, for an arbitrarily fixed base set of tree transformation classes, can decide the relationship concerning the inclusions between tree transformation classes obtained by composition from base classes. The method has numerous applications using different base sets of tree transformation classes, see [FülVág4], [FülVág5], [Fül2], [SluVág], and [GyeVág].

The subject of this thesis is the characterization of a new subtype of deterministic top-down tree transducers, called superlinear deterministic top-down tree transducers. We denote the class of superlinear deterministic top-down tree transformations by $s l-D T$. Superlinear deterministic top-down tree transducers are specialized linear deterministic top-down tree transducers and it holds that $s l-D T \subset l-D T$.

The concept of superlinear deterministic top-down tree transducers was proposed by H. Vogler during a personal communication with Z. Fülöp in 1992. It was motivated by the well known decomposition equation $D T=n d-H O M \circ l-D T$, which appeared first time in [Eng1] and [Bak3]. They discussed whether $l-D T$ in the above equation can be substituted by an even more restricted subclass of $D T$. It was guessed that a proper subclass of $l-D T$ would be suitable, namely the class $s l-D T$ of superlinear deterministic top-down tree transducers.

In this work we investigate properties of superlinear deterministic top-down tree transducers and the corresponding tree transformation class sl-DT. Although the starting point of the research was the decomposition equation $D T=$ $n d-H O M \circ s l-D T$, we have explored many other interesting results concerning superlinear deterministic top-down tree transducers and transformations. The problems we have arisen and answered were motivated by the earlier works regarding tree transducers (see, e.g., [Eng1], [Eng3], [Bak3], [FülVág1], [FülVág2]). These are as follows:

- What is the relationship between $s l-D T$ and the known tree transformation classes, such as DT or $l-D T$ ? In other words, how does the superlinear deterministic top-down tree transducers compare with the known types regarding transformational power?

- Is the class sl-DT closed under the composition?

- What kind of tree languages can be domain and range tree languages of superlinear deterministic top-down tree transformations?

- How can we characterize the compositions of $s l-D T$ with other known classes? 
The outline of the thesis is the following:

Chapter 1 We introduce the notions and notations, which are necessary to understand the results. Moreover, we also recall there some earlier results used in later chapters.

Chapter 2 We investigate certain properties of superlinear deterministic topdown tree transducers.

In Section 2.1 we prove that, among others, sl-DT is not closed under the composition, that $D T=n d-H O M \circ s l-D T$ holds, and that there is a total linear deterministic top-down tree transformation, which cannot be induced by consecutive application of a sequence of superlinear deterministic topdown tree transducers.

In Section 2.2 we show that the domains of superlinear deterministic topdown tree transformations are exactly those tree languages, which are recognized by tree recognizers of a new restricted type, called semi-universal deterministic top-down tree recognizers. Moreover, we prove that, for a deterministic recognizable tree language recognized by a given deterministic top-down tree recognizer, it is decidable whether this tree language can be domain of a superlinear deterministic top-down tree transformation.

Finally, in Section 2.3, it turns out that the class of ranges of superlinear deterministic top-down tree transformations is exactly the class of recognizable languages.

Chapter 3 We present two hierarchy theorems concerning superlinear deterministic top-down tree transformations, which show the uniqueness of this type in the family of deterministic top-down tree transformations. Namely, it turns out that, in contrast with the earlier types (e.g. DT, $l-D T$, etc.), the powers of the class sl-DT form a proper hierarchy. Roughly speaking, this means that the more superlinear deterministic top-down tree transducers we apply in succession, the higher transformational power we can get.

In Section 3.1 we prove that the hierarchy $\left\{s l-D T^{n} \mid n \geq 1\right\}$ is proper. More exactly, we prove a stronger statement, namely that $\left\{\operatorname{dom}\left(s l-D T^{n}\right) \mid n \geq 1\right\}$ is a proper hierarchy, where, for a tree transformation class $\mathcal{C}$, we denote by $\operatorname{dom}(\mathcal{C})$ the class of domains of the tree transformations in $\mathcal{C}$. Note that there are very few such hierarchy theorems in the literature, see [Eng3] and [FülVág2].

In Section 3.2 we consider total superlinear deterministic top-down tree transducers. Here we show that even the hierarchy $\left\{t-s l-D T^{n} \mid n \geq 1\right\}$ is proper.

Chapter 4 We explore how the class sl-DT behaves when composing with other known tree transformation classes. These other classes are HOM, l-DT, $n d-D T$, and $D T$. 
On behalf of this, we fix the set $M=\{H O M, s l-D T, l-D T, n d-D T, D T\}$ of tree transformation classes. Then we consider the monoid $[M]$ of all tree transformation classes of the form $X_{1} \circ \ldots \circ X_{m}$, where $m \geq 0$ and the $X_{i}$ 's are elements of $M$. For arbitrary composition classes $\mathcal{C}_{1}$ and $\mathcal{C}_{2}$ of the above form, we want to know whether some inclusion, equality, or incomparability holds between them. Clearly, it is enough if we can decide whether $\mathcal{C}_{1} \subseteq \mathcal{C}_{2}$ holds.

As the main result of the chapter, we give an effective description of the monoid $[M]$ with respect to the inclusion. This means that we present an algorithm, which can decide, given arbitrary two elements of the monoid, whether some inclusion holds between them.

The main steps of the development of this algorithm are as follows:

1. We consider the free monoid $M^{*}$ of strings generated by $M$. Then a unique homomorphism ||$: M^{*} \rightarrow[M]$ exists such that, for any $X_{1}, \ldots, X_{n} \in M,\left|X_{1} \cdot \ldots \cdot X_{n}\right|=X_{1} \circ \ldots \circ X_{n}$ (see [BurSan]). We denote the kernel of || by $\theta$, that is, for any strings $u, v \in M^{*}, u \theta v$ if and only if $|u|=|v|$.

2. We present a confluent and terminating rewriting system $R \subseteq M^{*} \times M^{*}$ such that $\Leftrightarrow_{R}^{*}=\theta$, where $\Leftrightarrow_{R}^{*}$ is the reflexive, symmetric, and transitive closure of the reduction relation $\Rightarrow_{R}$ over $M^{*}$.

3. We present the inclusion diagram, i.e. the Hasse diagram with respect to the inclusion of the set $|N F(R)|=\{|u| \mid u \in N F(R)\}$, where $N F(R)$ is the set of $R$-normal forms.

The algorithm works as follows. Given two arbitrary composition classes $\mathcal{C}=X_{1} \circ \ldots \circ X_{n}$ and $\mathcal{D}=Y_{1} \circ \ldots \circ Y_{m}$, we form the strings $x=X_{1} \cdot \ldots \cdot X_{n}$ and $y=Y_{1} \cdot \ldots \cdot Y_{m}$, and compute the corresponding $R$-normal forms $x \Rightarrow_{R}^{*} u$ and $y \Rightarrow_{R}^{*} v$. Since $R$ is terminating and confluent, $u$ and $v$ exist and unique. Moreover, $|x|=|u|$ and $|y|=|v|$ hold by $\Leftrightarrow_{R}^{*}=\theta$. Hence $\mathcal{C} \subseteq \mathcal{D}$ if and only if $|u| \subseteq|v|$. However, this latter can be decided by direct inspection of the inclusion diagram of $N F(R)$.

Finally, we summarize our results and mention some open problems regarding the superlinear property.

We note that the research has also resulted some by-products, which could be interesting for themself as well. These are the minimalization algorithm of deterministic top-down tree recognizers (see Subsection 1.4.4), the definition of semiuniversal deterministic top-down tree recognizers and that the minimalization preserves the semi-universal property (see Section 2.2), and the decomposition equation $D T=o p-n i-D T \circ n r-l-n d-H O M$ (see Lemma 2.1.9).

This thesis is strongly based on the papers [DánFül1], [DánFül2] and [Dán]. All results presented here appear in the above works. We shall refer to the corresponding paper at the beginning of each chapter. 
Acknowledgement. I am grateful to Zoltán Fülöp for his expert guidance and valuable suggestions during the course of this research.

I also wish to express my thanks to Pál Gyenizse and László Bernátsky for their very useful comments on a pre-release version of this thesis. 


\section{Chapter 1}

\section{Preliminaries}

In this chapter we introduce the notions and the notation, which are necessary for understanding.

Moreover, we recall some preliminary results used in later chapters. However, some of that results are referred to in a modified form (especially in Subsection 1.4.4), hence we present a proof, where it is necessary.

We note that the symbols $i, j, k, l, m$, and $n$ denotes integers in the sequel.

\subsection{Sets and relations}

An alphabet is a finite and nonempty set.

For arbitrary sets $A$ and $B$, we denote by $A \subseteq B$ that $A$ is a subset of $B$, by $A \subset B$ that $A$ is a proper subset of $B$, and by $A \bowtie B$ that neither $A \subseteq B$ nor $B \subseteq A$ hold.

We write $\operatorname{pow}(A)$ and $|A|$ for the power set and the cardinality of $A$, respectively. Moreover, $A \times B$ denotes the Cartesian product of $A$ and $B$.

A subset $X$ of $\operatorname{pow}(A)$ is called a partition of $A$ if $\emptyset \notin X, \cup_{P \in X} P=A$, and, for any $P_{1}, P_{2} \in X$ such that $P_{1} \neq P_{2}, P_{1} \cap P_{2}=\emptyset$ hold. In this case the elements of $X$ are also called classes of the partition $X$.

Given two sets $A$ and $B$, an arbitrary subset $\theta$ of $A \times B$ is called a relation from $A$ to $B$. We also write $a \theta b$ meaning that $(a, b) \in \theta$.

The inverse relation of $\theta$ is a relation $\theta^{-1}$ from $B$ to $A$, where, for any $a \in A$ and $b \in B, b \theta^{-1} a$ holds if and only if $a \theta b$.

For a subset $A^{\prime} \subseteq A$, we define the restriction of $\theta$ to $A^{\prime}$ as $\left.\theta\right|_{A^{\prime}}=\{(a, b) \in$ $\left.\theta \mid a \in A^{\prime}\right\}$.

A relation from $A$ to $A$ is called also a relation over $A$. The identity relation over $A$ is $\operatorname{id}(A)=\{(a, a) \mid a \in A\}$. A relation $\theta$ over a set $A$ is called

- reflexive if, for each $a \in A, a \theta a$ holds,

- transitive if, for any $a, b, c \in A, a \theta b$ and $b \theta c$ imply $a \theta c$, 
- symmetric if, for any $a, b \in A, a \theta b$ implies $b \theta a$,

- equivalence if it is reflexive, transitive, and symmetric,

- antisymmetric if, for any $a, b \in A, a \theta b$ and $b \theta a$ imply that $a$ and $b$ are identical, and

- partial order if it is reflexive, transitive, and antisymmetric.

An equivalence relation $\equiv$ over $A$ defines a partition of $A$, where, for any $a, b \in A, a$ and $b$ are in the same class if and only if $a \equiv b$. We denote by $[a]_{\equiv}$ the class of an element $a \in A$ with respect to $\equiv$.

A pair $(A, \leq)$ is called partially ordered set if $A$ is a set and $\leq$ is a partial order relation over $A$. A partially ordered set can be represented by a Hasse diagram (see $[$ BurSan]).

Let $H$ be a set of which the elements are classes and let $\subseteq$ be the inclusion relation over $H$. Clearly, $(H, \subseteq)$ is a partially ordered set. By the inclusion diagram of $H$ we mean the Hasse diagram of $(H, \subseteq)$.

Let $\theta$ be a relation from $A$ to $B$. For any $a \in A$, we put $\theta(a)=\{b \in B \mid a \theta b\}$. The sets $\operatorname{dom}(\theta)=\{a \in A \mid$ there is a $b \in B$ such that $a \theta b\}$ and range $(\theta)=\{b \in$ $B \mid$ there is an $a \in A$ such that $a \theta b\}$ are called the domain and the range of $\theta$, respectively. We say that $\theta$ is total if $\operatorname{dom}(\theta)=A$.

Let $\theta \subseteq A \times B$ and $\mu \subseteq B \times C$. The composition $\theta \circ \mu$ of the relations $\theta$ and $\mu$ is a relation from $A$ to $C$ defined as $\theta \circ \mu=\{(a, c) \mid$ there is a $b \in$ $B$ such that $a \theta b$ and $b \mu c\}$.

Let $\theta$ be a relation over $A$. The $n$-fold composition $\theta^{n}$ of $\theta$ with $n \geq 0$ is defined by $\theta^{0}=\mathrm{id}(A)$ and $\theta^{i}=\theta \circ \theta^{i-1}$, where $i>0$. The transitive closure and the reflexive and transitive closure of $\theta$ are the relations $\theta^{+}=\bigcup_{n>1} \theta^{n}$ and $\theta^{*}=\bigcup_{n>0} \theta^{n}$, respectively. Moreover, the symmetric closure of $\theta$ is $\theta \cup \theta^{-1}$.

We extend the concepts of domain, range, and composition for classes of relations. Let $\mathcal{C}$ and $\mathcal{C}^{\prime}$ be classes of relations. The domain and the range of $\mathcal{C}$ are defined by $\operatorname{dom}(\mathcal{C})=\{\operatorname{dom}(\theta) \mid \theta \in \mathcal{C}\}$ and range $(\mathcal{C})=\{$ range $(\theta) \mid \theta \in \mathcal{C}\}$, respectively. The composition of $\mathcal{C}$ and $\mathcal{C}^{\prime}$ is the relation class $\mathcal{C} \circ \mathcal{C}^{\prime}=\{\theta \circ \mu \mid \theta \in$ $\mathcal{C}$ and $\left.\mu \in \mathcal{C}^{\prime}\right\}$.

For an arbitrary class $\mathcal{C}$ of relations, we also define the $n$-fold composition $\mathcal{C}^{n}$ of $\mathcal{C}$ with $n \geq 1$ as $\mathcal{C}^{1}=\mathcal{C}$ and $\mathcal{C}^{i}=\mathcal{C} \circ \mathcal{C}^{i-1}$, where $i>1$. The transitive closure of $\mathcal{C}$ under the composition is the relation class $\mathcal{C}^{+}=\bigcup_{n \geq 1} \mathcal{C}^{n}$.

A class $\mathcal{C}$ of relations is said to be closed under the composition if $\mathcal{C}^{2} \subseteq \mathcal{C}$ holds.

Let $A$ and $B$ be sets. A relation $\nu \subseteq A \times B$ is called a mapping from $A$ to $B$, denoted by $\nu: A \rightarrow B$, if, for any $a \in A$, exactly one $b \in B$ exists such that $(a, b) \in \nu$ holds. In this case we also write $\nu(a)=b$ for $(a, b) \in \nu$.

A mapping $\nu$ from $A$ to $B$ is said to be

- injective if, for each $b \in B$, there is at most one $a \in A$ such that $\nu(a)=b$, 
- surjective if range $(\nu)=B$, and

- bijective if it is injective and surjective.

A bijective mapping is also called a bijection.

Finally, we introduce the concept of hierarchy. A family of classes $\left\{\mathcal{C}_{k} \mid k \geq 1\right\}$ is called a hierarchy if $\mathcal{C}_{k} \subseteq \mathcal{C}_{k+1}$ holds for each $k \geq 1$. Moreover, it is said to be proper if each inclusion is proper, i.e., for every $k \geq 1, \mathcal{C}_{k} \subset \mathcal{C}_{k+1}$ holds.

\section{$1.2 \quad$ Strings and string rewriting systems}

Let $A$ be an alphabet. A string $w$ over $A$ is a finite sequence $a_{1} \ldots a_{l}$, where $l \geq 0$ and $a_{1}, \ldots, a_{l} \in A$. Then the length of $w$, denoted by length $(w)$, is $l$. The empty string, i.e. the sequence containing no letter, is denoted by $e$. Thus length $(e)=0$. The set of all strings over $A$ is denoted by $A^{*}$.

Let $w=a_{1} \ldots a_{l}$ and $w^{\prime}=b_{1} \ldots b_{k}$ be strings over $A$. We define the concatenation $w_{1} w_{2}$ of $w_{1}$ and $w_{2}$ as the string $a_{1} \ldots a_{l} b_{1} \ldots b_{k}$ over $A$. If we want to refer to the concatenation explicitly as an operation over $A^{*}$, then we shall denote it by $\cdot$

We note that $A^{*}$ is the free monoid generated by $A$ with the concatenation operation, where $e$ is the identity (see [BurSan]).

An equivalence relation $\equiv$ over $A^{*}$ is called a congruence over $A^{*}$ if, for any words $u_{1}, u_{2}, v_{1}, v_{2} \in A^{*}, u_{1} \equiv u_{2}$ and $v_{1} \equiv v_{2}$ imply $u_{1} v_{1} \equiv u_{2} v_{2}$.

A string rewriting system $R$ over an alphabet $A$ is a finite relation over $A^{*}$. The elements of $R$ are called rewriting rules and we write $u \rightarrow v$ for $(u, v) \in R$.

The reduction relation $\Rightarrow_{R}$ over $A^{*}$ induced by $R$ is defined as follows. For any strings $w, w^{\prime} \in A^{*}, w \Rightarrow_{R} w^{\prime}$ holds if and only if a rule $v \rightarrow v^{\prime} \in R$ and strings $u_{1}, u_{2} \in A^{*}$ exist such that $w=u_{1} v u_{2}$ and $w^{\prime}=u_{1} v^{\prime} u_{2}$. We write $\Leftarrow_{R}$ for $\Rightarrow_{R}^{-1}$.

The reflexive, transitive, and symmetric closure of $\Rightarrow_{R}$, denoted by $\Leftrightarrow_{R}^{*}$, is a congruence over $A^{*}$. Informally speaking, $w \Leftrightarrow_{R}^{*} w^{\prime}$ holds if and only if there is a sequence $w_{0}, \ldots, w_{n}$ of strings, for some $n \geq 0$, such that $w_{0}=w, w_{n}=w^{\prime}$ and, for every $1 \leq i \leq n$, either $w_{i-1} \Rightarrow_{R} w_{i}$ or $w_{i} \Rightarrow_{R} w_{i-1}$ holds.

We say that a string rewriting system $R$ is

- terminating if there is no infinite sequence of the form $w_{1} \Rightarrow_{R} w_{2} \Rightarrow_{R} \ldots$ and

- confluent if, for any $v, w, w^{\prime} \in A^{*}, v \Rightarrow_{R}^{*} w$ and $v \Rightarrow_{R}^{*} w^{\prime}$ imply that a string $v^{\prime} \in A^{*}$ exists such that $w \Rightarrow_{R}^{*} v^{\prime}$ and $w^{\prime} \Rightarrow_{R}^{*} v^{\prime}$ hold.

A string $w \in A^{*}$ is called an $R$-normal form (or simply normal form if $R$ is understood) if there is no $w^{\prime} \in A^{*}$ such that $w \Rightarrow_{R} w^{\prime}$. The set of $R$-normal 
forms is denoted by $N F(R)$. A string $w^{\prime}$ is called a normal form of a string $w$ with respect to $R$ if $w \Rightarrow_{R}^{*} w^{\prime}$ and $w^{\prime}$ is an $R$-normal form.

We recall the following result (see $[\mathrm{Boo}]$ ):

Propositon 1.2.1 A terminating string rewriting system $R$ is confluent if and only if each word of $A^{*}$ has exactly one normal form.

Observe that the above statement can also be explained as follows. Considering the partition of $A^{*}$ with respect to the congruence $\Leftrightarrow_{R}^{*}$, a terminating $R$ is confluent if and only if each $\Leftrightarrow_{R^{-}}^{*}$ lass contains exactly one normal form.

We now mention a sufficient condition for a string rewriting system $R$ to be terminating.

A weight function is a mapping $\rho: A \rightarrow\{1,2, \ldots\}$, where $\rho(a)$ is called the weight of $a \in A$. Then $\rho$ can be extended to a mapping $\rho: A^{*} \rightarrow\{1,2, \ldots\}$ by letting $\rho(e)=0$ and, for every $w \in A^{*}$ and $a \in A, \rho(w a)=\rho(w)+\rho(a)$.

We say that $R$ is weight reducing if a weight function $\rho$ exists such that, for each rule $u \rightarrow v, \rho(u)>\rho(v)$ holds. It should be clear that a weight reducing string rewriting system is necessarily terminating.

For more information about string rewriting systems we refer the reader to [Boo], [BooOtt], and [Huet].

\subsection{Trees, tree languages, and tree transforma- tions}

A ranked alphabet $\Sigma$ is an alphabet, in which every symbol has a unique rank in the set of nonnegative integers. For each $m \geq 0$, the set of symbols in $\Sigma$

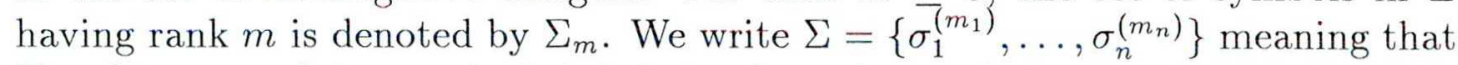
$\Sigma=\left\{\sigma_{1} \ldots, \sigma_{n}\right\}$ is a ranked alphabet, where the symbol $\sigma_{i}$ has the rank $m_{i}$, for each $1 \leq i \leq n$.

Let $\Sigma$ be a ranked alphabet. For a set $H$, the set of trees over $\Sigma$ indexed by $H$ is denoted by $T_{\Sigma}(H)$ and it is defined as the smallest set $U$ satisfying the following two conditions:

(i) $H \cup \Sigma_{0} \subseteq U$.

(ii) $\sigma\left(t_{1}, \ldots, t_{m}\right) \in U$, whenever $m>0, \sigma \in \Sigma_{m}$, and $t_{1}, \ldots, t_{m} \in U$.

The set $T_{\Sigma}(\emptyset)$ of ground trees over $\Sigma$ is written as $T_{\Sigma}$.

We specify a countable set $X=\left\{x_{1}, x_{2}, \ldots\right\}$ of symbols, called variables, and we put $X_{m}=\left\{x_{1}, \ldots, x_{m}\right\}$, for every $m \geq 0$. We assume that $X$ is disjoint to any ranked alphabet. We write $T_{\Sigma, m}$ for $T_{\Sigma}\left(X_{m}\right)$.

Trees can be represented as expressions with parentheses. For instance, if $\Sigma=\left\{\delta^{(2)}, \sigma^{(1)}, \#^{(0)}\right\}$ then $\delta(\sigma(\#), \#) \in T_{\Sigma}$ and $\delta\left(\delta\left(x_{1}, \#\right), \delta\left(\sigma\left(x_{2}\right), \sigma\left(x_{1}\right)\right)\right) \in$ $T_{\Sigma, 2}$. Moreover, these trees can be depicted as in Figure 1.1. 


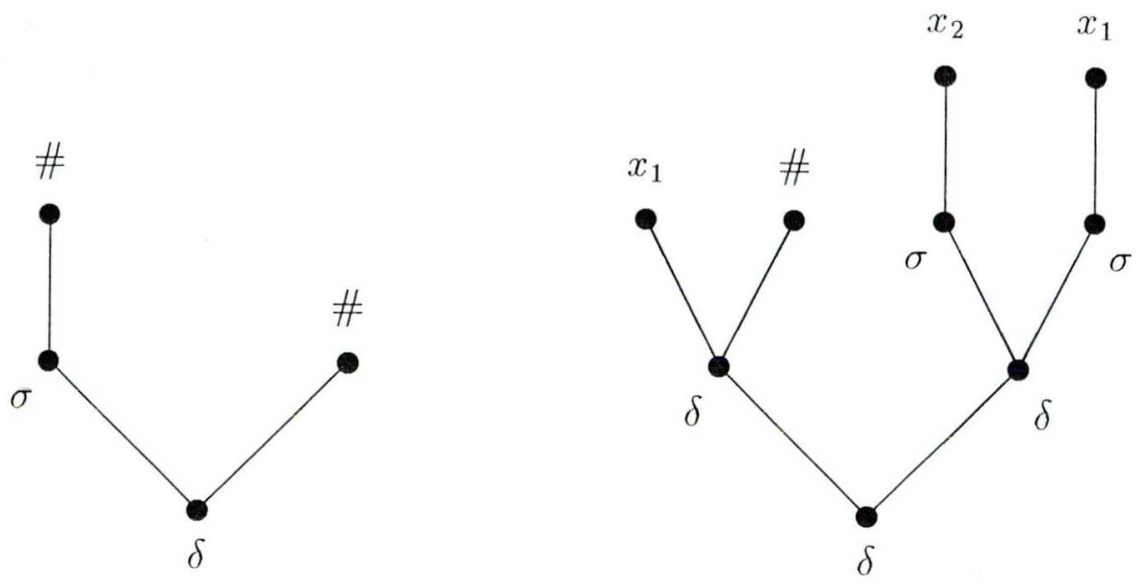

Figure 1.1: Representation of trees 
A "chain" tree, like $\sigma(\ldots \sigma(\#) \ldots)$, where $\sigma \in \Sigma_{1}$ occurs $i$ times, is abbreviated by $\sigma^{i}(\#)$. For example, $\sigma^{3}(\#)$ denotes the tree $\sigma(\sigma(\sigma(\#)))$.

We distinguish a subset $\hat{T}_{\Sigma, m}$ of $T_{\Sigma, m}$ as follows. A tree $t \in T_{\Sigma, m}$ is in $\hat{T}_{\Sigma, m}$ if and only if each variable in $X_{m}$ appears exactly once in $t$ and the order of the variables from left to right in $t$ is exactly $x_{1}, \ldots, x_{m}$. For instance, if $\Sigma=\left\{\sigma^{(2)}\right\}$, then $\sigma\left(x_{1}, x_{2}\right) \in \hat{T}_{\Sigma, 2}$, but $\sigma\left(x_{1}, x_{1}\right), \sigma\left(x_{2}, x_{1}\right) \notin \hat{T}_{\Sigma, 2}$.

Let $t \in T_{\Sigma, m}$, for some $m \geq 0$. We define the height of $t$, the set of subtrees of $t$, and the set of variables occurring in $t$, denoted by height $(t), \operatorname{sub}(t)$, and $\operatorname{var}(t)$, respectively, as follows.

(i) If $t=x_{i} \in X_{m}$, then height $(t)=0, \operatorname{sub}(t)=\left\{x_{i}\right\}$, and $\operatorname{var}(t)=\left\{x_{i}\right\}$.

(ii) If $t=\sigma \in \Sigma_{0}$, then height $(t)=0, \operatorname{sub}(t)=\{\sigma\}$, and $\operatorname{var}(t)=\emptyset$.

(iii) If $t=\sigma\left(t_{1}, \ldots, t_{n}\right)$, where $n>0, \sigma \in \Sigma_{n}$ and $t_{1}, \ldots, t_{n} \in T_{\Sigma, m}$, then height $(t)=1+\max \left\{\operatorname{height}\left(t_{i}\right) \mid 1 \leq i \leq n\right\}, \operatorname{sub}(t)=\{t\} \cup \bigcup_{1 \leq i \leq n} \operatorname{sub}\left(t_{i}\right)$, and $\operatorname{var}(t)=\bigcup_{1 \leq i \leq n} \operatorname{var}\left(t_{i}\right)$.

We note that if $t=\sigma\left(t_{1}, \ldots, t_{n}\right)$, where $n>0$, then, for any $1 \leq i \leq n, t_{i}$ is called the ith direct subtree of $t$.

We introduce the concept of tree substitution. Let $m \geq 0, t \in T_{\Sigma, m}$, and $s_{1}, \ldots, s_{m} \in S$ where $S$ is an arbitrary set of trees. We denote by $t\left[s_{1}, \ldots, s_{m}\right]$ the tree, which is obtained from $t$ by replacing each occurrence of $x_{i}$ in $t$ by $s_{i}$, for every $1 \leq i \leq m$. Clearly, $t\left[s_{1}, \ldots, s_{m}\right] \in T_{\Sigma}(S)$ holds.

Let $\Sigma$ be a ranked alphabet. A tree language $L$ over $\Sigma$ is an arbitrary subset $L \subseteq T_{\Sigma}$.

Let $\sigma \in \Sigma_{m}$, where $m \geq 1$, and let $L_{1}, \ldots, L_{m}$ be tree languages over $\Sigma$. The expression $\sigma\left(L_{1}, \ldots, L_{m}\right)$ denotes the tree language $\left\{\sigma\left(t_{1}, \ldots, t_{m}\right) \mid t_{1} \in\right.$ $\left.L_{1}, \ldots, t_{m} \in L_{m}\right\}$ over $\Sigma$.

Let $\Sigma$ and $\Delta$ be ranked alphabets. A tree transformation from $T_{\Sigma}$ to $T_{\Delta}$ is a relation from $T_{\Sigma}$ to $T_{\Delta}$. Since tree transformations are relations, the concepts of their domain, range and composition should be clear. Note that if $\tau \subseteq T_{\Sigma} \times T_{\Delta}$, then $\operatorname{dom}(\tau)$ and range $(\tau)$ are tree languages over $\Sigma$ and $\Delta$, respectively.

We specify the class $I=\left\{\operatorname{id}\left(T_{\Sigma}\right) \mid \Sigma\right.$ is a ranked alphabet $\}$ of identity tree transformations. Observe that if $I \subseteq \mathcal{C}$, then $\mathcal{C}^{2} \subseteq \mathcal{C}$ holds (i.e. $\mathcal{C}$ is closed under the composition) if and only if $\mathcal{C}^{2}=\mathcal{C}$.

Let $\mathcal{C}$ be a class of tree transformations. Now it is possible to define the reflexive and transitive closure of $\mathcal{C}$ as $\mathcal{C}^{*}=\bigcup_{n \geq 0} \mathcal{C}^{n}$, where $\mathcal{C}^{0}=I$.

\subsection{Top-down tree transducers}

A top-down tree transducer is a 5 -tuple $T=\left(Q, \Sigma, \Delta, q_{0}, R\right)$, where

- $Q$ is an unary ranked alphabet, meaning that $Q=Q_{1}$, called the set of states, such that $Q \cap(\Sigma \cup \Delta)=\emptyset$. 
- $\Sigma$ and $\Delta$ are ranked alphabets, called the input and the output ranked alphabet, respectively.

- $q_{0} \in Q$ is a distinguished element of $Q$, called the initial state.

- $R$ is a finite set of rules of the form

$$
q\left(\sigma\left(x_{1}, \ldots, x_{m}\right)\right) \rightarrow t\left[q_{1}\left(x_{i_{1}}\right), \ldots, q_{n}\left(x_{i_{n}}\right)\right],
$$

where $m, n \geq 0, \sigma \in \Sigma_{m}, 1 \leq i_{1}, \ldots, i_{n} \leq m, q, q_{1}, \ldots, q_{n} \in Q$, and $t \in \hat{T}_{\Delta, n}$.

A rule as above will be referred to as a $q$-rule for $\sigma$ or a $(q, \sigma)$-rule for brevity.

A rule of the form $(*)$ is said to be reducing if $t=x_{1}$ holds, i.e. it is of the form $q\left(\sigma\left(x_{1}, \ldots, x_{m}\right)\right) \rightarrow q^{\prime}\left(x_{i}\right)$, for some $q^{\prime} \in Q$ and $1 \leq i \leq m$.

The rules in $R$ induce a relation, called derivation and denoted by $\Rightarrow_{T}$, over the set $T_{\Delta}\left(Q\left(T_{\Sigma}\right)\right)$, where $Q\left(T_{\Sigma}\right)$ denotes the set $\left\{q(t) \mid q \in Q, t \in T_{\Sigma}\right\}$. It is defined as follows.

For any trees $r, s \in T_{\Delta}\left(Q\left(T_{\Sigma}\right)\right), r \Rightarrow_{T} s$ holds if and only if there is a rule $q\left(\sigma\left(x_{1}, \ldots, x_{m}\right)\right) \rightarrow t\left[q_{1}\left(x_{i_{1}}\right), \ldots, q_{n}\left(x_{i_{n}}\right)\right]$ in $\mathrm{R}$ such that $s$ can be obtained from $r$ by replacing an occurrence of a subtree $q\left(\sigma\left(t_{1}, \ldots, t_{m}\right)\right)$ of $r$ by

$$
t\left[q_{1}\left(t_{i_{1}}\right), \ldots, q_{n}\left(t_{i_{n}}\right)\right],
$$

where $t_{1}, \ldots, t_{m} \in T_{\Sigma}$.

The tree transformation $\tau_{T}$ induced by $T$ is defined as

$$
\tau_{T}=\left\{(r, s) \in T_{\Sigma} \times T_{\Delta} \mid q_{0}(r) \Rightarrow_{T}^{*} s\right\} .
$$

We note that top-down tree transducers are sometimes defined to have more than one initial states. However, that concept is not essentially different from our one. It is an easy exercise to show that, for each top-down tree transducer having more initial states, a top-down tree transducer with one initial state can be constructed, which induces the same tree transformation.

We say that $T$ is deterministic if, for any $q \in Q$ and $\sigma \in \Sigma$, there is at most one $(q, \sigma)$-rule in $R$. The expression deterministic top-down tree transducer is abbreviated to dt tree transducer in the sequel.

A tree transformation $\tau$ is called a $d t$ tree transformation if a dt tree transducer $T$ exists so that $\tau=\tau_{T}$. The class of all $\mathrm{dt}$ tree transformations is denoted by DT.

We suppose that $T$ is deterministic in what follows.

Consider an arbitrary $(q, \sigma)$-rule in $R$ of the above $(*)$ form. The term $t\left[q_{1}\left(x_{i_{1}}\right), \ldots, q_{n}\left(x_{i_{n}}\right)\right]$ is called the right-hand side of the rule and it is denoted by $\operatorname{rhs}(q, \sigma)$.

We note that the order of the variables from left to right occurring in the above right-hand side is $x_{i_{1}}, \ldots, x_{i_{n}}$, because the order of the ones from left to right occurring in $t$ is $x_{1}, \ldots, x_{n}$. 
Again, consider an arbitrary $(q, \sigma)$-rule in $R$ of the $(*)$ form. For each $1 \leq$ $j \leq m$, we define $\operatorname{rst}(q, \sigma, j)=\left\{q_{k} \in Q \mid 1 \leq k \leq n, i_{k}=j\right\}$, i.e. the set of states applied to $x_{j}$ in $\operatorname{rhs}(q, \sigma)$.

\subsubsection{Restricted types}

We introduce some restricted subtypes of dt tree transducers applying certain restrictions to the form of rules. Moreover, we specify a unique abbreviation for the name of each type. Finally, we define a way to construct combined types using the ones listed bellow.

We note that the types (1), (2), (4), and (8) are well known from the theory of tree transducers (see, e.g., [GécSte4]). Moreover, (5), (6), and (7) were also defined in [AndBos].

Let $T=\left(Q, \Sigma, \Delta, q_{0}, R\right)$ be a dt tree transducer. We say that $T$ is:

(1) Total (t) if, for any $\sigma \in \Sigma$ and $q \in Q$, there exists a $(q, \sigma)$-rule in $R$. Note that, being $T$ deterministic, this implies that there is exactly one $(q, \sigma)$-rule in $R$, for any $q \in Q$ and $\sigma \in \Sigma$. Clearly, in this case $\tau_{T}$ is a total tree transformation.

(2) Linear (1) if, for every rule $q\left(\sigma\left(x_{1}, \ldots, x_{m}\right)\right) \rightarrow t\left[q_{1}\left(x_{i_{1}}\right), \ldots, q_{n}\left(x_{i_{n}}\right)\right]$ in $R$, each of the variables $x_{1}, \ldots, x_{m}$ appears at most once in the right-hand side. Note that in this case $m \geq n$.

(3) Superlinear (sl) if it is linear and, for every $\sigma \in \Sigma_{m}$ with $m \geq 0$ and any two different states $q, q^{\prime} \in Q$, $\operatorname{var}(\operatorname{rhs}(q, \sigma)) \cap \operatorname{var}\left(\operatorname{rhs}\left(q^{\prime}, \sigma\right)\right)=\emptyset$ holds. Equivalently, $T$ is sl-dt, if it is linear and, for every $\sigma \in \Sigma_{m}$ with $m \geq 0$ and $1 \leq i \leq m$, there is at most one state $q \in Q$ such that $x_{i}$ occurs in $\operatorname{rhs}(q, \sigma)$.

(4) Nondeleting (nd) if, for every $q\left(\sigma\left(x_{1}, \ldots, x_{m}\right)\right) \rightarrow t\left[q_{1}\left(x_{i_{1}}\right), \ldots, q_{n}\left(x_{i_{n}}\right)\right]$ in $R$, each of the variables $x_{1}, \ldots, x_{m}$ appears at least once in the right-hand side. Note that in this case $m \leq n$.

(5) Order preserving (op) if, for every $q\left(\sigma\left(x_{1}, \ldots, x_{m}\right)\right) \rightarrow t\left[q_{1}\left(x_{i_{1}}\right), \ldots, q_{n}\left(x_{i_{n}}\right)\right]$ in $R$, the order $i_{1} \leq \ldots \leq i_{n}$ holds.

(6) Nonreducing (nr) if $R$ does not contain reducing rule, i.e. there is no rule of the form $q\left(\sigma\left(x_{1}, \ldots, x_{m}\right)\right) \rightarrow q^{\prime}\left(x_{i}\right)$, where $1 \leq i \leq m$ in $R$.

(7) Nonincreasing (ni) if, for every $q\left(\sigma\left(x_{1}, \ldots, x_{m}\right)\right) \rightarrow t\left[q_{1}\left(x_{i_{1}}\right), \ldots, q_{n}\left(x_{i_{n}}\right)\right]$ in $R$, it holds that height $(t) \leq 1$. Note that in this case either $t=x_{1}$ or $t=\delta\left(x_{1}, \ldots, x_{n}\right)$, for some $\delta \in \Delta_{n}$. 
(8) Relabeling $(\mathrm{rl})$, if each rule in $R$ is of the form

$$
q\left(\sigma\left(x_{1}, \ldots, x_{m}\right)\right) \rightarrow \delta\left(q_{1}\left(x_{1}\right), \ldots, q_{m}\left(x_{m}\right)\right),
$$

where $m \geq 0, \sigma \in \Sigma_{m}, \delta \in \Delta_{m}$. Roughly speaking, processing a tree, $T$ does not change the skeleton, only relabels the nodes.

(9) Homomorphism (hom) if it is total and $Q$ is a singleton set, i.e. $Q=\left\{q_{0}\right\}$.

These attributes can be combined. For instance, by an op-l-nd-dt tree transducer, we mean an order preserving, linear and nondeleting deterministic topdown tree transducer.

Let $x$ be an arbitrary combination of some of the modifiers in $\{t, 1, s l$, nd, op, nr, ni, rl, hom $\}$ such as l-nd, op-ni-sl, etc. A dt tree transformation is said to be an $\mathrm{x}$-dt tree transformation if it can be induced by an $\mathrm{x}$ - $\mathrm{dt}$ tree transducer.

Observe that the relabeling deterministic top-down tree transducers are exactly the nonincreasing, nonreducing, linear, and nondeleting deterministic topdown tree transducers, hence $\mathrm{rl}$ is in fact a shorthand for the combination ni-nrl-nd.

The class of all $\mathrm{x}$-dt tree transformations is denoted by $x$-DT. Clearly, the order of the modifiers in a combination is irrelevant from the point of view of meaning, i.e. if $\mathrm{x}$ is a combination of modifiers and $\mathrm{y}$ is a permutation of $\mathrm{x}$ then $x-D T=y$-DT holds. Moreover, we can assume without loss of generality that any modifier occurs at most once in a combination.

We write simply hom instead of hom-dt. For example, op-l-nd-hom means an order preserving, linear and nondeleting homomorphism dt tree transducer.

Let $\mathrm{x}$ be a combination of modifiers as above. The class of all $\mathrm{x}$-hom tree transformations is denoted by $x-H O M$.

We note that, for any combination $\mathrm{x}$, both $I \subseteq x-D T$ and $I \subseteq x-H O M$ hold. Observe that if $\mathcal{C}$ and $\mathcal{D}$ are tree transformation classes and $I \subseteq \mathcal{D}$, then $\mathcal{C} \subseteq \mathcal{C} \circ \mathcal{D}$. This follows from the fact that every tree transformation $\tau$ in $\mathcal{C}$ can be decomposed as $\tau=\tau \circ \iota$, where $\iota$ is a suitable identity in $\mathcal{D}$. Specially, $x-D T^{n} \subseteq x-D T^{n+1}$ and $x-H O M^{n} \subseteq x-H O M^{n+1}$ hold for every $n \geq 0$.

\subsubsection{Compositions and decompositions}

In this subsection we introduce the concept of syntactic composition of $\mathrm{dt}$ tree transducers. Moreover, we clarify the correspondence between compositions of dt tree transformations and syntactic compositions of dt tree transducers. Finally, we recall some earlier results concerning compositions of dt tree transformation classes.

Let $T=\left(Q, \Sigma, \Delta, q_{0}, R\right)$ and $T^{\prime}=\left(Q^{\prime}, \Delta, \Omega, q_{0}^{\prime}, R^{\prime}\right)$ be dt tree transducers. By the syntactic composition of $T$ and $T^{\prime}$ we mean the dt tree transducer

$$
T \circ T^{\prime}=\left(Q^{\prime} \times Q, \Sigma, \Omega,\left(q_{0}^{\prime}, q_{0}\right), R^{\prime \prime}\right),
$$


where $R^{\prime \prime}$ is the smallest set of rules, which satisfies the following condition. If

(1) there is a rule $q\left(\sigma\left(x_{1}, \ldots, x_{m}\right)\right) \rightarrow t\left[q_{1}\left(x_{i_{1}}\right), \ldots, q_{n}\left(x_{i_{n}}\right)\right] \in R$ and

(2) there is a state $p \in Q^{\prime}$ such that $p(t) \Rightarrow_{T^{\prime}}^{*} t^{\prime}\left[p_{1}\left(x_{j_{1}}\right), \ldots, p_{k}\left(x_{j_{k}}\right)\right]$, for some $t^{\prime} \in \hat{T}_{\Omega, k}$ with $k \geq 0$ and $p_{1}, \ldots, p_{k} \in Q^{\prime}$,

then the rule

$$
(p, q)\left(\sigma\left(x_{1}, \ldots, x_{m}\right)\right) \rightarrow t^{\prime}\left[\left(p_{1}, q_{j_{1}}\right)\left(x_{i_{j_{1}}}\right), \ldots,\left(p_{k}, q_{j_{k}}\right)\left(x_{i_{j_{k}}}\right)\right]
$$

is in $R^{\prime \prime}$. It is easy to see that $R^{\prime \prime}$ is finite. Roughly speaking, the composition transducer works such that

(1) applies the appropriate $(q, \sigma)$-rule of $T$ and

(2) lets $T^{\prime}$ process the tree $t$, obtained by the application of the above rule, starting with state $p$.

Reducing rules play a very important role in the characterization of the syntactic composition of sl-dt tree transducers, hence we pay more attention for them.

Suppose that there is a reducing rule in $R$, i.e. a rule of the form

$$
q\left(\sigma\left(x_{1}, \ldots, x_{m}\right)\right) \rightarrow q^{\prime}\left(x_{i}\right),
$$

where $m \geq 1$ and $1 \leq i \leq m$. Clearly, for every $p \in Q^{\prime}, p\left(x_{1}\right) \Rightarrow_{T^{\prime}}^{*} p\left(x_{1}\right)$ holds. Therefore the rule

$$
(p, q)\left(\sigma\left(x_{1}, \ldots, x_{m}\right)\right) \rightarrow\left(p, q^{\prime}\right)\left(x_{i}\right)
$$

is in $R^{\prime \prime}$. Informally speaking, a reducing rule for $\sigma$ in $R$ as above induces $\left|Q^{\prime}\right|$ different reducing rules for $\sigma$ in $R^{\prime \prime}$ such that each of them contains $x_{i}$ in the right-hand side.

Now we show the correspondence between syntactic compositions of dt tree transducers and compositions of dt tree transformations. The following results implicitly appear in [Bak3] and [Eng1], but in the present form they are stated in [FülVág1].

Propositon 1.4.1 For any $d t$ tree transducers $T$ and $T^{\prime}$, the following statements hold:

(1) If $T$ is total or $T^{\prime}$ is nondeleting, then $\tau_{T \circ T^{\prime}}=\tau_{T} \circ \tau_{T^{\prime}}$.

(2) $\left.\tau_{T \circ T^{\prime}}\right|_{\operatorname{dom}\left(\tau_{T}\right)}=\tau_{T} \circ \tau_{T^{\prime}}$.

(3) Let $x$ be any of the modifiers $\{t, l, n d$, hom $\}$. If both $T$ and $T^{\prime}$ are $x$-dt tree transducers, then $T \circ T^{\prime}$ is also $x-d t$. 
We do not present the formal proofs of the above statements. However, it is worth to recall informally the main points of them.

To prove (1) and (2), it is enough to see that $T \circ T^{\prime}$ processes every tree on almost the same way, as the consecutive application of $T$ and $T^{\prime}$ does. The only difference is the following.

Suppose that $\tau_{T}$ is partial and $T^{\prime}$ is not nondeleting. Then there is a tree $t$ on which $T$ fails, i.e. $t \notin \operatorname{dom}\left(\tau_{T}\right)$ and hence $t \notin \operatorname{dom}\left(\tau_{T} \circ \tau_{T^{\prime}}\right)$. However, since $T^{\prime}$ is not nondeleting, it can occur that, informally speaking, the rules of the dt tree transducer $T \circ T^{\prime}$ deletes the subtrees of $t$, on which $T$ fails, without processing. Thus $t \in \operatorname{dom}\left(\tau_{T \text { o } T^{\prime}}\right)$ can hold.

Clearly, this implies $\tau_{T} \circ \tau_{T^{\prime}} \subseteq \tau_{T \circ T^{\prime}}$ and that the converse inclusion generally does not hold.

As for (3), it is straightforward to show, by the construction of the rules of $T \circ T^{\prime \prime}$.

A large series of equations concerning compositions of dt tree transformation classes can be derived from Proposition 1.4.1. We present a short list of them containing that ones, which are necessary in our proofs.

\section{Corollary 1.4 .2}

$\begin{array}{ll}\text { (1) } t-D T \circ D T & =D T \\ \text { (2) } D T \circ n d-D T & =D T \\ \text { (3) } n d-H O M \circ n d-H O M & =n d-H O M \\ \text { (4) } n d-H O M \circ D T & =D T \\ \text { (5) } D T \circ n d-H O M & =D T \\ \text { (6) } n r-l-n d-H O M \circ D T & =D T \\ \text { (7) } n d-H O M \circ n d-D T & =n d-D T\end{array}$

Characterizing a class of $\mathrm{dt}$ tree transformations, it is always a pivot question whether it is closed under the composition. By Proposition 1.4.1, it is easy to show that the classes $t-D T$ and $n d-D T$ are.

On the other hand, it has turned out that the tree transformation classes DT and $l-D T$ are not closed under the composition, i.e. $D T \subset D T^{2}$ and $l-D T \subset$ $l-D T^{2}$ (see [Rou] or [GécSte4]).

In this case the question naturally arises whether the powers of these classes constitute proper hierarchies. The following proposition, cited from [FülVág1], shows that they do not.

Propositon 1.4.3 For any $n \geq 2$, it holds that $D T^{n}=D T^{2}$ and $l-D T^{n}=$ $l-D T^{2}$.

Finally, we recall a decomposition equation from [Bak3], which shows that every dt tree transformation can be induced by consecutive application of an ndhom and an 1-dt tree transducer. Note that this result also appears in [GécSte4].

Propositon 1.4.4 DT $=n d-H O M \circ l-D T$ 


\subsubsection{Top-down tree recognizers and recognizable tree languages}

A top-down tree recognizer $(t t r) T=\left(Q, \Sigma, \Sigma, q_{0}, R\right)$ is a top-down tree transducer, of which the rules are of the form

$$
q\left(\sigma\left(x_{1}, \ldots, x_{m}\right)\right) \rightarrow \sigma\left(q_{1}\left(x_{1}\right), \ldots, q_{m}\left(x_{m}\right)\right),
$$

where $m \geq 0, \sigma \in \Sigma_{m}$, and $q, q_{1}, \ldots, q_{m} \in Q$. If $T$ is deterministic, then it is called a deterministic top-down tree recognizer (dttr).

Observe that $\tau_{T} \subseteq \operatorname{id}\left(T_{\Sigma}\right)$ holds, i.e. $\tau_{T}$ is a partial identity over $T_{\Sigma}$. Moreover, if $T$ is deterministic, then it is an rl-dt tree transducer.

Let $T=\left(Q, \Sigma, \Sigma, q_{0}, R\right)$ be a dttr. We say that a state $q \in Q$ is universal, if, for all $t \in T_{\Sigma}, q(t) \Rightarrow_{T}^{*} t$ holds, i.e. $\left\{t \in T_{\Sigma} \mid q(t) \Rightarrow_{T}^{*} t\right\}=T_{\Sigma}$. Observe that, for any rule $q\left(\sigma\left(x_{1}, \ldots, x_{m}\right)\right) \rightarrow \sigma\left(q_{1}\left(x_{1}\right), \ldots, q_{m}\left(x_{m}\right)\right) \in R$, if $q$ is universal, then $q_{1}, \ldots, q_{m}$ are necessarily universal, too.

We say that $T$ recognizes the tree $t \in T_{\Sigma}$ if $q_{0}(t) \Rightarrow_{T}^{*} t$. The tree language recognized by $T$ is $L(T)=\left\{t \in T_{\Sigma} \mid q_{0}(t) \Rightarrow_{T}^{*} t\right\}$. Observe that $L(T)=\operatorname{dom}\left(\tau_{T}\right)$.

A tree language is recognizable (resp. deterministic recognizable) if it is recognized by a ttr (resp. dttr). We denote by $R E C$ (resp. DREC) the class of recognizable (resp. deterministic recognizable) tree languages.

Note that the original concept of recognizability concerning tree languages is defined by descending (or bottom-up) tree automata, see in [GécSte4]. However, consulting Chapter II in [GécSte4], one can easily see that top-down tree recognizers are equivalent to regular tree grammars in normal form and hence to descending tree automata.

Clearly, $D R E C \subseteq R E C$ holds. Moreover, it is a well-known result (see, e.g., [GécSte4]) that the inclusion is proper, i.e. $D R E C \subset R E C$.

\subsubsection{Minimal deterministic top-down tree recognizers}

Deterministic top-down tree recognizers also have automaton type equivalent, namely deterministic ascending (or top-down) tree automata (dtta). A short reflection will show that there are mainly notational differences between these types of devices, hence we can apply the notions and results in [GécSte3] to dttr's without difficulties.

An $n$-ary dtta is a 5 -tuple $A=\left(Q, \Sigma, Y_{n}, q_{0}, F\right)$, where $n>0$,

- $Q$ is the finite nonempty set of states,

- $q_{0} \in Q$ is the initial state,

- $F=\left(Q_{1}, \ldots, Q_{n}\right) \in(\operatorname{pow}(Q))^{n}$ is the final state vector, 
- $Y_{n}=\left\{y_{1}, \ldots, y_{n}\right\}$ is the set of automaton variables, and

- $\Sigma$ is a ranked alphabet, where $\Sigma \cap Y_{n}=\emptyset, \Sigma_{0}=\emptyset$, and every $\sigma \in \Sigma_{m}$ with $m>0$ is realized as a mapping $\sigma^{A}: Q \rightarrow Q^{m}$.

We now specify how a $\operatorname{dtta} A$ recognizes trees. Define the mapping $\alpha_{A}$ : $T_{\Sigma}\left(Y_{n}\right) \rightarrow \operatorname{pow}(Q)$ as follows.

(i) $\alpha_{A}\left(y_{i}\right)=Q_{i}$, for $1 \leq i \leq n$, and

(ii) $\alpha_{A}(t)=\left\{q \in Q \mid \sigma^{A}(q) \in \alpha_{A}\left(t_{1}\right) \times \ldots \times \alpha_{A}\left(t_{m}\right)\right\}$, if $t=\sigma\left(t_{1}, \ldots, t_{m}\right)$ with $m>0, \sigma \in \Sigma_{m}$, and $t_{1}, \ldots, t_{m} \in T_{\Sigma}\left(Y_{n}\right)$.

The tree language recognized by $A$ is $L(A)=\left\{t \in T_{\Sigma}\left(Y_{n}\right) \mid q_{0} \in \alpha_{A}(t)\right\}$.

We show that, for any dttr, an equivalent dtta can be constructed.

Construction 1.4.5 Consider an arbitrary dttr $T=\left(Q, \Sigma, \Sigma, q_{0}, R\right)$ and suppose that $\Sigma_{0}=\left\{\delta_{1}, \ldots, \delta_{n}\right\}$ with $n>0$. Let $p \notin Q$ be a new state. Define the dtta $A=\left(Q \cup\{p\}, \Sigma-\Sigma_{0}, \Sigma_{0}, q_{0}, F\right)$, where

- $F=\left(Q_{1}, \ldots, Q_{n}\right)$ with $Q_{i}=\left\{q \in Q \mid q\left(\delta_{i}\right) \rightarrow \delta_{i} \in R\right\}$, for $1 \leq i \leq n$, and,

- for all $m>0, \sigma \in \Sigma_{m}$, and $q \in Q \cup\{p\}$, if $q \in Q$ and $q\left(\sigma\left(x_{1}, \ldots, x_{m}\right)\right) \rightarrow$ $\sigma\left(q_{1}\left(x_{1}\right), \ldots, q_{m}\left(x_{m}\right)\right)$ is in $R$, then let $\sigma^{A}(q)=\left(q_{1}, \ldots, q_{m}\right)$, otherwise let $\sigma^{A}(q)=(p, \ldots, p)$.

It is straightforward to prove $L(A)=L(T)$. Note that the case $n=0$ is trivial, because then $L(T)=\emptyset$.

Conversely, for any dtta, an equivalent dttr can be constructed.

Construction 1.4.6 Let $A=\left(Q, \Sigma, Y_{n}, q_{0}, F\right)$ be an arbitrary dtta. Assign the rank 0 to each element of $Y_{n}$ and let $\Delta=\Sigma \cup Y_{n}$. Define the dttr $T=$ $\left(Q, \Delta, \Delta, q_{0}, R\right)$, where $R$ is constructed as follows:

(i) for all $1 \leq i \leq n, q\left(y_{i}\right) \rightarrow y_{i} \in R$ if and only if $q \in Q_{i}$ and,

(ii) for all $m>0, \sigma \in \Sigma_{m}$ and $q, q_{1}, \ldots, q_{m} \in Q$, the rule $q\left(\sigma\left(x_{1}, \ldots, x_{m}\right)\right) \rightarrow$ $\sigma\left(q_{1}\left(x_{1}\right), \ldots, q_{m}\left(x_{m}\right)\right)$ is in $R$ if and only if $\sigma^{A}(q)=\left(q_{1}, \ldots, q_{m}\right)$.

It is easy to show $L(T)=L(A)$.

We now recall some definitions and results from [GécSte3] using the dttr notation. Note that two dttr's are called equivalent if they recognize the same tree language.

Let $T=\left(Q, \Sigma, \Sigma, q_{0}, R\right)$ be a dttr. A state $q \in Q$ of $T$ is called 0 -state if the set $\left\{t \in T_{\Sigma} \mid q(t) \Rightarrow_{T}^{*} t\right\}$ is empty.

A $\operatorname{dttr} T=\left(Q, \Sigma, \Sigma, q_{0}, R\right)$ is said to be normalized, if either it has no 0 -state, or the only 0 -state is $q_{0}$ and in this case $Q=\left\{q_{0}\right\}$ and $R=\emptyset$ hold. 
We note that this concept of normalization differs from the original one in [GécSte3] on page 40. Namely, if a dttr is normalized by the above definition, then the dtta given by the Construction 1.4.5 is normalized in the sense of [GécSte3]. However, the converse is not true, that is if a dtta is normalized in the sense of [GécSte3], then the dttr given by the Construction 1.4.6 is generally not normalized by the above definition.

The difference follows from the fact that in the case of dtta's every $\sigma \in \Sigma_{m}$ with $m>0$ is realized as a mapping $\sigma^{A}: Q \rightarrow Q^{m}$, hence $\sigma^{A}$ is defined for each $q \in Q$. That is why the 0 -states cannot be discarded completely from the state set of a normalized dtta. On the other hand, the 0 -states (except $q_{0}$ ) and the corresponding rules can be deleted without difficulties in the case of dttr's, as it is shown by the following proposition.

Propositon 1.4.7 For any dttr $T=\left(Q, \Sigma, \Sigma, q_{0}, R\right)$, an equivalent normalized dttr $T_{\text {nor }}=\left(Q^{\prime}, \Sigma, \Sigma, q_{0}, R^{\prime}\right)$ can be constructed effectively such that $Q^{\prime} \subseteq Q$ and $R^{\prime} \subseteq R$.

Proof. The set of non 0-states can be computed as follows. Define a sequence $Q^{(0)} \subseteq Q^{(1)} \subseteq \ldots$ of subsets of $Q$, where

(i) $Q^{(0)}=\left\{q \in Q \mid \exists \delta \in \Sigma_{0}: q(\delta) \rightarrow \delta \in R\right\}$ and,

(ii) for $i \geq 0, Q^{(i+1)}=Q^{(i)} \cup\left\{q \in Q \mid \exists m \geq 1, \sigma \in \Sigma_{m}: q\left(\sigma\left(x_{1}, \ldots, x_{m}\right)\right) \rightarrow\right.$ $\sigma\left(q_{1}\left(x_{1}\right), \ldots, q_{m}\left(x_{m}\right)\right) \in R$ and $\left.q_{1}, \ldots, q_{m} \in Q^{(i)}\right\}$.

Obviously, there is a $k \geq 0$ such that $Q^{(k)}=Q^{(k+j)}$, for every $j \geq 1$.

If $q_{0} \notin Q^{(k)}$, then let $Q^{\prime}=\left\{q_{0}\right\}$ and $R^{\prime}=\emptyset$. Clearly, in this case $L(T)=$ $L\left(T_{\text {nor }}\right)=\emptyset$ holds.

Finally, if $q_{0} \in Q^{(k)}$, then let $Q^{\prime}=Q^{(k)}$ and $R^{\prime}=\left\{q\left(\sigma\left(x_{1}, \ldots, x_{m}\right)\right) \rightarrow\right.$ $\left.\sigma\left(q_{1}\left(x_{1}\right), \ldots, q_{m}\left(x_{m}\right)\right) \in R \mid q, q_{1}, \ldots, q_{m} \in Q^{(k)}\right\}$. Observe that, for any $t \in L(T)$, during the derivation $q_{0}(t) \Rightarrow_{T}^{*} t$ only such rules are applied, which do not contain a 0 -state, hence $q_{0}(t) \Rightarrow_{T_{\text {nor }}}^{*} t$, too. Therefore $L\left(T_{\text {nor }}\right)=L(T)$.

We define the binary relation $\mapsto_{T}$ over $Q$ as follows. Let $q, p \in Q$, then $q \mapsto_{T} p$ if and only if there exists a $\sigma \in \Sigma_{m}$ with $m>0$ such that $p$ appears in $\operatorname{rhs}(q, \sigma)$. We say that $p$ is accessible from $q$ if $q \mapsto_{T}^{*} p$ holds. The $\operatorname{dttr} T$ is called connected if every state in $Q$ is accessible from $q_{0}$.

Note that the above concept of accessibility is derived from the concept of reachability of states of dtta's defined in [GécSte3] on pages 41-42.

Propositon 1.4.8 For any $d t t r T=\left(Q, \Sigma, \Sigma, q_{0}, R\right)$, an equivalent connected dttr $T_{\text {con }}=\left(Q^{\prime}, \Sigma, \Delta, q_{0}, R^{\prime}\right)$ can be constructed effectively such that $Q^{\prime} \subseteq Q$ and $R^{\prime} \subseteq R$. Moreover, if $T$ is normalized, then $T_{\text {con }}$ is also normalized. 
Proof. The set of accessible states can be determined in the following way. Define a sequence $Q^{(0)} \subseteq Q^{(1)} \subseteq \ldots$ of subsets of $Q$, where

(i) $Q^{(0)}=\left\{q_{0}\right\}$ and,

(ii) for $i \geq 0, Q^{(i+1)}=Q^{(i)} \cup\left\{q \in Q \mid \exists m \geq 1, \sigma \in \Sigma_{m}, p \in Q^{(i)}: q\right.$ occurs in $\operatorname{rhs}(p, \sigma)\}$.

Clearly, there is a $k \geq 0$ such that $Q^{(k)}=Q^{(k+j)}$, for every $j \geq 1$.

Let $Q^{\prime}=Q^{(k)}$ and $R^{\prime}=\left\{q\left(\sigma\left(x_{1}, \ldots, x_{m}\right)\right) \rightarrow \sigma\left(q_{1}\left(x_{1}\right), \ldots, q_{m}\left(x_{m}\right)\right) \in R \mid q\right.$, $\left.q_{1}, \ldots, q_{m} \in Q^{(k)}\right\}$. It is easy to show that $L\left(T_{\text {con }}\right)=L(T)$.

Note that the construction of $Q^{\prime}$ is derived from the procedure computing $H_{k}$ 's in [GécSte3] on page 43.

Moreover, by the construction of $T_{\text {con }}$, it should be obvious that if $T$ is normalized, then $T_{\text {con }}$ is also normalized.

By Proposition 1.4.8, if $T$ is a normalized dttr, then $T_{c o n}$ is also normalized. However, the converse is not true, i.e. if $T$ is a connected dttr, then $T_{\text {nor }}$ is not necessarily connected. To see this, consider the following example.

Let $T=\left(\left\{q_{0}, q_{1}, q_{2}\right\}, \Sigma, \Sigma, q_{0}, R\right)$, where $\Sigma=\left\{\sigma^{(2)}, \#^{(0)}\right\}$ and $R=\left\{q_{0}(\#) \rightarrow\right.$ $\left.\#, q_{1}(\#) \rightarrow \#, q_{0}\left(\sigma\left(x_{1}, x_{2}\right)\right) \rightarrow \sigma\left(q_{1}\left(x_{1}\right), q_{2}\left(x_{2}\right)\right)\right\}$. Clearly, $T$ is a connected dttr, but $T_{\text {nor }}=\left(\left\{q_{0}, q_{1}\right\}, \Sigma, \Sigma, q_{0},\left\{q_{0}(\#) \rightarrow \#, q_{1}(\#) \rightarrow \#\right\}\right)$ is not connected, because $q_{1}$ is not an accessible state in $T_{\text {nor }}$.

If we refer to the construction of the normalized and connected equivalent dttr $T_{\text {nor,con }}$ of a dttr $T$ in the sequel, then we always mean that $T_{\text {nor }}$ must be determined first from $T$ as defined in the proof of Proposition 1.4.7, and $T_{\text {nor,con }}$ must be computed from $T_{\text {nor }}$ as specified in the proof of Proposition 1.4.8.

A dttr $T$ is said to be minimal if, for every dttr $T^{\prime}$ such that $T^{\prime}$ is equivalent to $T,|Q| \leq\left|Q^{\prime}\right|$ holds, where $Q$ and $Q^{\prime}$ are the sets of states of $T$ and $T^{\prime}$, respectively (cf. minimal dtta on page 38 in [GécSte3]).

Let $T=\left(Q, \Sigma, \Sigma, q_{0}, R\right)$ and $T^{\prime}=\left(Q^{\prime}, \Sigma, \Sigma, q_{0}^{\prime}, R^{\prime}\right)$ be dttr's. We say that $T$ and $T^{\prime}$ are isomorphic if there exists a bijection $\nu: Q \rightarrow Q^{\prime}$ such that $\nu\left(q_{0}\right)=q_{0}^{\prime}$ holds and, for any $m \geq 0, \sigma \in \Sigma_{m}$, and states $q, q_{1}, \ldots, q_{m} \in Q$, the rule $q\left(\sigma\left(x_{1}, \ldots, x_{m}\right)\right) \rightarrow \sigma\left(q_{1}\left(x_{1}\right), \ldots, q_{m}\left(x_{m}\right)\right)$ is in $R$ if and only if the rule $\nu(q)\left(\sigma\left(x_{1}, \ldots, x_{m}\right)\right) \rightarrow \sigma\left(\nu\left(q_{1}\right)\left(x_{1}\right), \ldots, \nu\left(q_{m}\right)\left(x_{m}\right)\right)$ is in $R^{\prime}$. In this case $\nu$ is also called a dttr isomorphism (cf. dtta isomorphism on page 39 in [GécSte3]). Note that if $T$ and $T^{\prime}$ are isomorphic, then clearly $|Q|=\left|Q^{\prime}\right|$ and they are equivalent.

We say that a minimal dttr $T$ is unique up to isomorphism if, for each minimal $\operatorname{dttr} T^{\prime}$, which is equivalent to $T$, it holds that $T^{\prime}$ and $T$ are isomorphic.

The following result is derived from Theorem 8 in [GécSte3].

Propositon 1.4.9 For any dttr $T$, an equivalent minimal dttr $T_{\min }$ exists. Moreover, it is unique up to isomorphism and can effectively be constructed. 
We also present the construction of the minimal dttr. We note that the following construction is derived from the construction of reduced dtta presented in [GécSte3] on page 43.

Construction 1.4.10 Let $T=\left(Q, \Sigma, \Sigma, q_{0}, R\right)$ be a dttr. By Propositions 1.4.7 and 1.4.8, we can assume without loss of generality that $T$ is normalized and connected (if it is not, then consider $T_{n o r, c o n}$ instead of $T$ ). We define a sequence $\equiv_{0} \supseteq \equiv_{1} \supseteq \ldots$ of equivalence relations over $Q$, where

(i) $q \equiv_{0} p$ if and only if, for every $\sigma \in \Sigma_{0}, q(\sigma) \rightarrow \sigma \in R$ holds if and only if $p(\sigma) \rightarrow \sigma \in R$ and,

(ii) for $i \geq 0, q \equiv_{i+1} p$ if and only if $q \equiv_{i} p$ and, for every $\sigma \in \Sigma_{m}$ with $m>0$, either both $q$ and $p$ are not defined on $\sigma$, or both $q\left(\sigma\left(x_{1}, \ldots, x_{m}\right)\right) \rightarrow$ $\sigma\left(q_{1}\left(x_{1}\right), \ldots, q_{m}\left(x_{m}\right)\right)$ and $p\left(\sigma\left(x_{1}, \ldots, x_{m}\right)\right) \rightarrow \sigma\left(p_{1}\left(x_{1}\right), \ldots, p_{m}\left(x_{m}\right)\right)$ are in $R$ and then $q_{j} \equiv_{i} p_{j}$ holds, for each $1 \leq j \leq m$.

Clearly, there is a $k \geq 0$ such that $\equiv_{k}$ and $\equiv_{k+j}$ are the same, for every $j \geq 1$. The minimal dttr equivalent to $T$ is defined as $T_{\min }=\left(Q^{\prime}, \Sigma, \Sigma,\left[q_{0}\right]_{\equiv_{k}}, R^{\prime}\right)$, where

- $Q^{\prime}=\left\{[q]_{\equiv_{k}} \mid q \in Q\right\}$ and

- $[q]_{\equiv_{k}}\left(\sigma\left(x_{1}, \ldots, x_{m}\right)\right) \rightarrow \sigma\left(\left[q_{1}\right]_{\equiv_{k}}\left(x_{1}\right), \ldots,\left[q_{m}\right]_{\equiv_{k}}\left(x_{m}\right)\right) \in R^{\prime}$ if and only if $q\left(\sigma\left(x_{1}, \ldots, x_{m}\right)\right) \rightarrow \sigma\left(q_{1}\left(x_{1}\right), \ldots, q_{m}\left(x_{m}\right)\right) \in R$, for any $m \geq 0, \sigma \in \Sigma_{m}$, and $q, q_{1}, \ldots, q_{m} \in Q$.

The proof of Proposition 1.4.9 is rather long and needs new concepts to introduce (e.g. dttr congruence, quotient dttr, etc.). However, it is an easy exercise to present it if one follows the proof of Theorem 8 in [GécSte3] step by step. Hence we omit the proof here.

However, we note that, proving Proposition 1.4.9 on the basis of [GécSte3], it should be considered that, in contrast with a dtta, a state of a dttr is not necessarily defined for all input symbols (cf. definitions of $\equiv_{i+1}$ in (ii) of Construction 1.4.10 and $\rho_{k+1}$ in (ii) in [GécSte3] on page 43).

\subsubsection{Domain and range tree languages}

In this subsection we investigate domain and range tree languages of various types of $\mathrm{dt}$ tree transformations from the point of view of recognizability.

Recall that a dttr is also an $\mathrm{rl}$-dt tree transducer, hence $D R E C \subseteq \operatorname{dom}(r l-D T)$ holds. On the other hand, the following statement shows that $\operatorname{dom}(D T) \subseteq$ $D R E C$. The original statement can be found as Lemma 5 in [FülVág1] (this result also appears in [Eng2]), although it is slightly modified here for our purposes. 
Propositon 1.4.11 For any $d t$ tree transducer $T=\left(Q, \Sigma, \Delta, q_{0}, R\right)$, a connected dttr $T^{\prime}=\left(Q^{\prime}, \Sigma, \Sigma,\left\{q_{0}\right\}, R^{\prime}\right)$ exists such that $L\left(T^{\prime}\right)=\operatorname{dom}\left(\tau_{T}\right)$.

Proof. We define a sequence $Q^{(0)} \subseteq Q^{(1)} \subseteq \ldots$ of subsets of pow $(Q)$ and a sequence $R^{(0)} \subseteq R^{(1)} \subseteq \ldots$ of finite sets of rules of the form $P\left(\sigma\left(x_{1}, \ldots, x_{m}\right)\right) \rightarrow$ $\sigma\left(P_{1}\left(x_{1}\right), \ldots, P_{m}\left(x_{m}\right)\right)$, where $m \geq 0, \sigma \in \Sigma_{m}, P, P_{1}, \ldots, P_{m} \subseteq Q$ :

(i) Put $P_{0}=\left\{q_{0}\right\}$. Let $Q^{(0)}=\left\{P_{0}\right\} \cup\left\{\operatorname{rst}\left(q_{0}, \sigma, j\right) \mid m \geq 1, \sigma \in \Sigma_{m}, 1 \leq j \leq\right.$ $m, q_{0}$ is defined on $\sigma$ in $\left.R\right\}$. Moreover, let $R^{(0)}=\left\{P_{0}\left(\sigma\left(x_{1}, \ldots, x_{m}\right)\right) \rightarrow\right.$ $\sigma\left(\operatorname{rst}\left(q_{0}, \sigma, 1\right)\left(x_{1}\right), \ldots, \operatorname{rst}\left(q_{0}, \sigma, m\right)\left(x_{m}\right)\right) \mid m \geq 0, \sigma \in \Sigma_{m}, q_{0}$ is defined on $\sigma$ in $R\}$.

(ii) Let $k \geq 0$. For any $P \in Q^{(k)}$ and $\sigma \in \Sigma$, we say that $P$ is defined on $\sigma$ if, for each $q \in P, q$ is defined on $\sigma$ in $R$ and in this case if $\sigma \in \Sigma_{m}$ with $m \geq 1$, we put $S_{P, \sigma, j}=\bigcup_{q \in P} \operatorname{rst}(q, \sigma, j)$, for every $1 \leq j \leq m$. Specially, $P=\emptyset$ is defined for all $\sigma \in \Sigma$ and if $\sigma \in \Sigma_{m}$ with $m \geq 1$, then $S_{\emptyset, \sigma, j}=\emptyset$, for each $1 \leq j \leq m$.

Now let $Q^{(k+1)}=Q^{(k)} \cup\left\{S_{P, \sigma, j} \mid m \geq 1, \sigma \in \Sigma_{m}, 1 \leq j \leq m, P \in Q^{(k)}\right.$, $P$ is defined on $\sigma\}$. Moreover, let $R^{(k+1)}=R^{(k)} \cup\left\{P\left(\sigma\left(x_{1}, \ldots, x_{m}\right)\right) \rightarrow\right.$ $\sigma\left(S_{P, \sigma, 1}\left(x_{1}\right), \ldots, S_{P, \sigma, m}\left(x_{m}\right)\right) \mid m \geq 0, \sigma \in \Sigma_{m}, P \in Q^{(k)}, P$ is defined on $\sigma\}$. Specially, if $\emptyset \in Q^{(k)}$, then $\emptyset\left(\sigma\left(x_{1}, \ldots, x_{m}\right)\right) \rightarrow \sigma\left(\emptyset\left(x_{1}\right), \ldots, \emptyset\left(x_{m}\right)\right) \in$ $R^{(k+1)}$, for every $m \geq 0$ and $\sigma \in \Sigma_{m}$.

Clearly, a $k \geq 0$ exists such that $Q^{(k+1)}=Q^{(k)}$, and then $Q^{(k+1)}=Q^{(k+2)}=\ldots$ and $R^{(k+1)}=R^{(k+2)}=\ldots$ hold. Let $Q^{\prime}=Q^{(k+1)}$ and $R^{\prime}=R^{(k+1)}$.

It is easy to see that $T^{\prime}$ is exactly the connected version of the dttr defined in the proof of Lemma 5 in [FülVág1].

Observe that if $T$ is linear, then $Q^{\prime}$ in the proof of Proposition 1.4.11 consists of sets containing at most one element. Moreover, if $\emptyset \in Q^{\prime}$, then it is a universal state of $T^{\prime}$.

We have $\operatorname{dom}(r l-D T)=\operatorname{dom}(l-D T)=\operatorname{dom}(D T)=D R E C$. Moreover, we recall that $D R E C=\operatorname{dom}\left(D T^{n}\right)=\operatorname{dom}\left(l-D T^{n}\right)$ for every $n \geq 1$, see [FülVág3].

As for ranges of various types of $\mathrm{dt}$ tree transformation classes, we recall the following results.

It is well known and easy to show that range $(n d-D T)$ is not recognizable and hence range $(D T)$ is also not recognizable. To see this, consider the following example.

Let $T=\left(Q, \Sigma, \Delta, q_{0}, R\right)$ be an nd-dt tree transducer, where

- $Q=\left\{q_{0}, q_{1}\right\}$,

- $\Sigma=\left\{\sigma^{(1)}, \#^{(0)}\right\}$, 
- $\Delta=\left\{\delta^{(2)}, \sigma^{(1)}, \#^{(0)}\right\}$, and

- $R=\left\{q_{0}\left(\sigma\left(x_{1}\right)\right) \rightarrow \delta\left(q_{1}\left(x_{1}\right), q_{1}\left(x_{1}\right)\right), q_{1}\left(\sigma\left(x_{1}\right)\right) \rightarrow \sigma\left(q_{1}\left(x_{1}\right)\right), q_{1}(\#) \rightarrow \#\right\}$.

Then range $\left(\tau_{T}\right)=\left\{\delta\left(\sigma^{n}(\#), \sigma^{n}(\#)\right) \mid n \geq 0\right\}$, which is obviously not recognizable.

On the other hand, by Corollary 6.6 of Chapter IV in [GécSte4], it holds that range $(l-D T) \subseteq R E C$. We note that even the equality can be shown, i.e. range $(l-D T)=R E C$. This result is proved in Section 2.3 . 


\section{Chapter 2}

\section{Properties of sl-dt tree transducers}

In this chapter we investigate certain properties of superlinear deterministic topdown tree transducers and tree transformations induced by them.

In Section 2.1 we explore some basic properties, e.g., that $s l-D T$ is not closed under the compositions and that $D T=n d-H O M \circ s l-D T$ holds. The results of this section appear in Section 3 of [DánFül1].

In Sections 2.2 and 2.3 we investigate domain and range tree languages of superlinear deterministic top-down tree transformations, respectively. We define a new type of top-down tree recognizers, called semi-universal deterministic topdown tree recognizer. We show that the domain tree languages of superlinear deterministic top-down tree transducers are exactly that ones, which are recognized by semi-universal deterministic top-down tree recognizers. On the basis of this result, we develop a decision algorithm, which decides whether an arbitrary deterministic recognizable tree language can be domain of a superlinear deterministic top-down tree transformation. Moreover, we prove that the range tree languages of superlinear deterministic top-down tree transducers are exactly the recognizable tree languages. We note that the results of Sections 2.2 and 2.3 were published in [Dán].

\subsection{Basic properties}

In this section we describe some basic properties of superlinear deterministic top-down tree transducers and the class sl-DT.

First we present two simple observations.

Observation 2.1.1 Let $T=(\{q\}, \Sigma, \Delta, q, R)$ be a hom tree transducer. Then $T$ is superlinear if and only if it is linear. 
Proof. By definition, if $T$ is superlinear, then it is also linear. Conversely, let $T$ be linear. Since $T$ has the only state $q$, for any $\sigma \in \Sigma_{m}$ with $m \geq 0$ and integer $i$ with $1 \leq i \leq m$, there is at most one rule for $\sigma$ such that $x_{i}$ occurs in $\operatorname{rhs}(q, \sigma)$. Hence $T$ is superlinear as well.

Corollary 2.1.2 l-HOM $=s l-H O M$

The second observation immediately follows from the definitions of nondeleting and superlinear top-down tree transducers.

Observation 2.1.3 Let $T=\left(Q, \Sigma, \Delta, q_{0}, R\right)$ be a nd-sl-dt tree transducer. For every symbol $\sigma \in \Sigma_{n}$ with $n \geq 1$, there is at most one state $q \in Q$ such that $R$ contains a $(q, \sigma)$-rule.

In the following theorem, we show that there exist two sl-dt tree transformations such that their composition is not even a dt tree transformation. This implies that the tree transformation class $s l-D T$ is not closed under the composition.

Theorem 2.1.4 sl-DT $T^{2}-D T \neq \emptyset$

Proof. Consider the ranked alphabets $\Sigma=\{\sigma, \#\}$ and $\Delta=\{\#\}$, where $\sigma$ and \# have rank 2 and 0 , respectively. Define the sl-dt tree transducers $T_{1}$ and $T_{2}$ in the following way.

Let $T_{1}=\left(Q_{1}, \Sigma, \Sigma, p, R_{1}\right)$ where

- $Q_{1}=\{p, q\}$ and

- $R$ consists of the rules $p\left(\sigma\left(x_{1}, x_{2}\right)\right) \rightarrow \sigma\left(q\left(x_{1}\right), q\left(x_{2}\right)\right)$ and $q(\#) \rightarrow \#$.

Moreover, let $T_{2}=\left(\{p\}, \Sigma, \Delta, p,\left\{p\left(\sigma\left(x_{1}, x_{2}\right)\right) \rightarrow \#\right\}\right)$. It is easy to show that $\tau_{T_{1}} \circ \tau_{T_{2}}=\{(\sigma(\#, \#), \#)\}$.

Suppose that a dt tree transducer $T=\left(Q, \Sigma, \Delta, q_{0}, R\right)$ exists such that $\tau_{T}=$ $\tau_{T_{1}} \circ \tau_{T_{2}}$. Since $\Delta=\Delta_{0}$, the only $\left(q_{0}, \sigma\right)$-rule in $R$ must be of one of the following three forms:

(1) $q_{0}\left(\sigma\left(x_{1}, x_{2}\right)\right) \rightarrow \#$.

(2) $q_{0}\left(\sigma\left(x_{1}, x_{2}\right)\right) \rightarrow q\left(x_{1}\right)$, for some $q \in Q$.

(3) $q_{0}\left(\sigma\left(x_{1}, x_{2}\right)\right) \rightarrow q\left(x_{2}\right)$, for some $q \in Q$.

We show that each case leads to a contradiction.

Indeed, in case $(1)$, for every $t_{1}, t_{2} \in T_{\Sigma},\left(\sigma\left(t_{1}, t_{2}\right), \#\right) \in \tau_{T}$ holds, which is a contradiction. 
In case $(2), q(\#) \rightarrow \#$ must be in $R$, because $(\sigma(\#, \#), \#) \in \tau_{T}$. However, in this case $(\sigma(\#, t), \#) \in \tau_{T}$, for every $t \in T_{\Sigma}$, which is a contradiction again.

Similarly, (3) is also impossible. We obtained that a suitable $T$ does not exist. Therefore $\tau_{T_{1}} \circ \tau_{T_{2}} \notin D T$.

We note that, since $s l-D T$ is a subclass of $l-D T$ and hence of $D T$, the above result also shows that neither DT nor $l-D T$ is closed under the composition. (However, these results are already known, see, e.g., [FülVág1].)

The property superlinear may seem to be a very strict restriction. Nevertheless, the following lemma and theorem show that the composition of $n d-H O M$ and $s l-D T$ yields $D T$.

Lemma 2.1.5 $l-D T \subseteq n d-H O M \circ s l-D T$

Proof. Let $T=\left(Q, \Sigma, \Delta, q_{0}, R\right)$ be an 1-dt tree transducer. We define an nd-hom tree transducer $H=\left(\{p\}, \Sigma, \Sigma^{\prime}, p, R^{\prime}\right)$ and an sl-dt tree transducer $T^{\prime}=$ $\left(Q, \Sigma^{\prime}, \Delta, q_{0}, R^{\prime \prime}\right)$ such that $\tau_{T}=\tau_{H} \circ \tau_{T^{\prime}}$.

To this end, let $p_{1}, \ldots, p_{n}$ be a fixed enumeration of the states in $Q$. Define $\Sigma^{\prime}$ to be the smallest ranked alphabet, for which $\Sigma_{k n}^{\prime}=\left\{\sigma^{\prime} \mid \sigma \in \Sigma_{k}\right\}$, for every $k \geq 0$. Then let $R^{\prime}$ be the smallest set of rules satisfying the following condition. For every $k \geq 0$ and $\sigma \in \Sigma_{k}$, let the rule

$$
p\left(\sigma\left(x_{1}, \ldots, x_{k}\right)\right) \rightarrow \sigma^{\prime}(\overbrace{p\left(x_{1}\right), \ldots, p\left(x_{1}\right)}^{n}, \ldots, \overbrace{p\left(x_{k}\right), \ldots, p\left(x_{k}\right)}^{n})
$$

be in $R^{\prime}$. (Note that $x_{i}$ occurs $n$ times in $\operatorname{rhs}(p, \sigma)$, for each $1 \leq i \leq k$ ).

Finally, let $R^{\prime \prime}$ be the set of rules constructed as follows. For each $r \in R$, carry on the following procedure.

Assume that $r$ is of the form $q\left(\sigma\left(x_{1}, \ldots, x_{k}\right)\right) \rightarrow t$, for some $q \in Q, k \geq 0$, and $\sigma \in \Sigma_{k}$. Suppose that $q=p_{i}$, for some $1 \leq i \leq n$. Then let the rule $q\left(\sigma^{\prime}\left(x_{1}, \ldots, x_{k n}\right)\right) \rightarrow t^{\prime}$ be in $R^{\prime \prime}$, where the tree $t^{\prime}$ is obtained from $t$ in the way that, for every $1 \leq j \leq k$, we substitute $x_{j}$ by $x_{(j-1) n+i}$ in $t$. (More formally, we put $t^{\prime}=t\left[x_{i}, x_{n+i}, \ldots, x_{(k-1) \cdot n+i}\right]$.) Let $R^{\prime \prime}$ consist of only these rules.

We show that $T^{\prime}$ is superlinear. Since $T$ is linear and, by the above construction, different variables in the right-hand side of a rule in $R$ are substituted by different ones, $T^{\prime}$ is certainly linear.

Moreover, consider a $\left(q, \sigma^{\prime}\right)$-rule and a $\left(q^{\prime}, \sigma^{\prime}\right)$-rule from $R^{\prime \prime}$, where $\sigma^{\prime} \in \Sigma_{k n}^{\prime}$, for some $k \geq 0$, and $q, q^{\prime} \in Q$ are different states. Suppose that the state $q$ is the $i$ th one and the state $q^{\prime}$ is the $j$ th one in the sequence $p_{1}, \ldots, p_{n}$. Obviously, $i \neq j$ and $1 \leq i, j \leq n$. Then, by the construction of $R^{\prime \prime}, \operatorname{var}\left(\operatorname{rhs}\left(q, \sigma^{\prime}\right)\right) \subseteq$ $\left\{x_{i}, x_{n+i}, \ldots, x_{(k-1) n+i}\right\}$ and $\operatorname{var}\left(\operatorname{rhs}\left(q^{\prime}, \sigma^{\prime}\right)\right) \subseteq\left\{x_{j}, x_{n+j}, \ldots, x_{(k-1) n+j}\right\}$. By $i \neq j$,

$$
\left\{x_{i}, x_{n+i}, \ldots, x_{(k-1) n+i}\right\} \cap\left\{x_{j}, x_{n+j}, \ldots, x_{(k-1) n+j}\right\}=\emptyset
$$

holds, that is $\operatorname{var}\left(\operatorname{rhs}\left(q, \sigma^{\prime}\right)\right) \cap \operatorname{var}\left(\operatorname{rhs}\left(q^{\prime}, \sigma^{\prime}\right)\right)=\emptyset$. Hence $T^{\prime}$ is superlinear. 
To prove that $\tau_{T}=\tau_{H} \circ \tau_{T^{\prime}}$ holds, it is enough to show the following equivalence. For every $s \in T_{\Sigma}, t \in T_{\Delta}$ and $q \in Q, q(s) \Rightarrow_{T}^{*} t$ if and only if an $s^{\prime} \in T_{\Sigma^{\prime}}$ exists such that $p(s) \Rightarrow_{H}^{*} s^{\prime}$ and $q\left(s^{\prime}\right) \Rightarrow_{T^{\prime}}^{*} t$. However, this is straightforward to show by induction on the height of $s$, hence we do not present the formal proof.

We can easily derive the following two very important results from Lemma 2.1.5. The first one shows that any $\mathrm{dt}$ tree transformation can be substituted by the consecutive application of an nd-dt and an sl-dt tree transducer, cf. Proposition 1.4.4.

Theorem 2.1.6 DT $=$ nd-HOM osl-DT

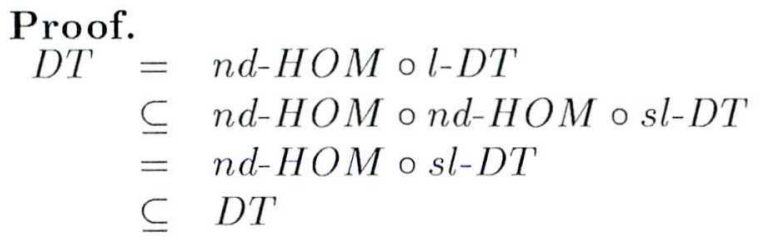

Proof.

(by Proposition 1.4.4)

(by Lemma 2.1.5)

(by (3) of Corollary 1.4.2)

(by Proposition 1.4.4)

By Proposition 1.4.3, any tree transformation, given by the composition of an arbitrary sequence of deterministic top-down tree transformations, can be substituted by the consecutive application of two appropriate dt tree transducers.

The following result show that, informally speaking, the subsequent application of an nd-hom and two sl-dt tree transducers has the same transformation power.

Corollary 2.1.7 $D T^{2}=n d-H O M \circ s l-D T^{2}$

$$
\begin{aligned}
\begin{array}{l}
\text { Proof. } \\
D T^{2}
\end{array} & =D T \circ n d-H O M \circ s l-D T \\
& =D T \circ s l-D T \\
& =n d-H O M \circ s l-D T^{2}
\end{aligned}
$$

(by Theorem 2.1.6)

(by Theorem 2.1.6)

By definition, $\operatorname{var}(\operatorname{rhs}(q, \sigma)) \cap \operatorname{var}\left(\operatorname{rhs}\left(q^{\prime}, \sigma\right)\right)=\emptyset$, for any $(q, \sigma)-$, and $\left(q^{\prime}, \sigma\right)-$ rules of an sl-dt tree transducer $T=\left(Q, \Sigma, \Delta, q_{0}, R\right)$, if $q \neq q^{\prime}$.

In the following technical lemma we show that this property is hereditary for trees $r$ and $r^{\prime}$ with variables, which are obtained by derivation from the same tree $s$ starting with some different states $q$ and $q^{\prime}$, respectively.

Lemma 2.1.8 Let $T=\left(Q, \Sigma, \Delta, q_{0}, R\right)$ be an sl-dt tree transducer. Let $s \in \hat{T}_{\Sigma, k}$, for some $k \geq 1$, and let $q, q^{\prime} \in Q$ be different states such that the derivations $q(s) \Rightarrow_{T}^{*} r$ and $q^{\prime}(s) \Rightarrow_{T}^{*} r^{\prime}$ hold for some trees $r, r^{\prime} \in T_{\Delta}\left(Q\left(X_{k}\right)\right)$. Then $\operatorname{var}(r) \cap$ $\operatorname{var}\left(r^{\prime}\right)=\emptyset$ if and only if $s \neq x_{1}$. 
Proof. In other words, our lemma states that, for every nontrivial tree $s \in$ $\hat{T}_{\Sigma, k}$ with $k \geq 1$ and for every $1 \leq i \leq k$, there is at most one state $q \in Q$ such that $x_{i}$ occurs in $r$, where $r$ is defined by $q(s) \Rightarrow_{T}^{*} r$.

Clearly, if $s=x_{1}$ then $r=q\left(x_{1}\right)$ and $r^{\prime}=q^{\prime}\left(x_{1}\right)$, hence $\operatorname{var}(r) \cap \operatorname{var}\left(r^{\prime}\right)=$ $\left\{x_{1}\right\} \neq \emptyset$.

Now let $s \neq x_{1}$, that is $s=\sigma\left(s_{1}, \ldots, s_{m}\right)$, for some $m \geq 0, \sigma \in \Sigma_{m}$, and $s_{1}, \ldots, s_{m} \in T_{\Sigma, k}$. Then the derivations $q(s) \Rightarrow_{T}^{*} r$ and $q^{\prime}(s) \Rightarrow_{T}^{*} r^{\prime}$ can be detailed as

$$
q(s)=q\left(\sigma\left(s_{1}, \ldots, s_{m}\right)\right) \underset{T}{\Rightarrow} t\left[q_{1}\left(s_{i_{1}}\right), \ldots, q_{n}\left(s_{i_{n}}\right)\right] \underset{T}{\stackrel{*}{\Rightarrow}} t\left[t_{1}, \ldots, t_{n}\right]=r
$$

and

$$
q^{\prime}(s)=q^{\prime}\left(\sigma\left(s_{1}, \ldots, s_{m}\right)\right) \underset{T}{\Rightarrow} t^{\prime}\left[p_{1}\left(s_{j_{1}}\right), \ldots, p_{l}\left(s_{j_{l}}\right)\right] \underset{T}{\stackrel{*}{\Rightarrow}} t^{\prime}\left[t_{1}^{\prime}, \ldots, t_{l}^{\prime}\right]=r^{\prime}
$$

respectively, where the rules

$$
q\left(\sigma\left(x_{1}, \ldots, x_{m}\right)\right) \rightarrow t\left[q_{1}\left(x_{i_{1}}\right), \ldots, q_{n}\left(x_{i_{n}}\right)\right]
$$

and

$$
q^{\prime}\left(\sigma\left(x_{1}, \ldots, x_{m}\right)\right) \rightarrow t^{\prime}\left[p_{1}\left(x_{j_{1}}\right), \ldots, p_{l}\left(x_{j_{l}}\right)\right]
$$

are in $R$.

Since $s \in \hat{T}_{\Sigma, k}$, it holds that $\operatorname{var}\left(s_{i}\right) \cap \operatorname{var}\left(s_{j}\right)=\emptyset$, whenever $1 \leq i \neq j \leq m$. On the other hand, by the superlinear property of $T$, we have $\left\{i_{1}, \ldots, i_{n}\right\} \cap$ $\left\{j_{1}, \ldots, j_{l}\right\}=\emptyset$.

Consequently, we have $\operatorname{var}\left(s_{i_{u}}\right) \cap \operatorname{var}\left(s_{j_{v}}\right)=\emptyset$ for every integer $1 \leq u \leq n$ and $1 \leq v \leq l$. Moreover, any variable occurring in $t_{u}$ (resp. $t_{v}^{\prime}$ ) also occurs in $s_{i_{u}}$ (resp. $\left.s_{j_{v}}^{\prime}\right)$. Therefore, we also have $\operatorname{var}\left(t_{u}\right) \cap \operatorname{var}\left(t_{v}^{\prime}\right)=\emptyset$, which proves $\operatorname{var}(r) \cap \operatorname{var}\left(r^{\prime}\right)=\emptyset$.

The following decomposition lemma plays a very important role in the proof of the main result of Section 3.1.

Lemma 2.1.9 DT = op-ni-DT onr-l-nd-HOM

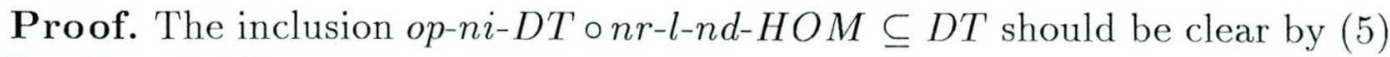
of Corollary 1.4.2.

To prove the converse let $T=\left(Q, \Sigma, \Delta, q_{0}, R\right)$ be an arbitrary dt tree transducer. We show that an op-ni-dt tree transducer $T^{\prime}$ and an nr-l-nd-hom tree transducer $T^{\prime \prime}$ exist so that $\tau_{T}=\tau_{T^{\prime}} \circ \tau_{T^{\prime \prime}}$.

We construct the op-ni-dt tree transducer $T^{\prime}=\left(Q, \Sigma, \Sigma^{\prime}, q_{0}, R^{\prime}\right)$ and the nr-1nd-hom tree transducer $T^{\prime \prime}=\left(\{p\}, \Sigma^{\prime}, \Delta, p, R^{\prime \prime}\right)$ in the following way. Define the ranked alphabet $\Sigma^{\prime}$ such that

$$
\Sigma_{n}^{\prime}=\left\{\sigma^{q} \mid q\left(\sigma\left(x_{1}, \ldots, x_{m}\right)\right) \rightarrow t\left[q_{1}\left(x_{i_{1}}\right), \ldots, q_{n}\left(x_{i_{n}}\right)\right] \in R \text { and } t \neq x_{1}\right\},
$$


for every $n \geq 0$.

Then define the sets $R^{\prime}$ and $R^{\prime \prime}$ of rules as the smallest sets satisfying the following conditions. Let $q\left(\sigma\left(x_{1}, \ldots, x_{m}\right)\right) \rightarrow t\left[q_{1}\left(x_{i_{1}}\right), \ldots, q_{n}\left(x_{i_{n}}\right)\right]$ be a rule in $R$, where $t \neq x_{1}$. Let $j_{1}, \ldots, j_{n}$ be a permutation of the numbers $1, \ldots, n$, for which $i_{j_{1}} \leq \ldots \leq i_{j_{n}}$. (Clearly, at least one such permutation exists. If there are more possibilities, then fix one.) Let $k_{1}, \ldots, k_{n}$ be the inverse of the permutation $j_{1}, \ldots, j_{n}$. Hence $j_{k_{l}}=l$ holds for each $l$ such that $1 \leq l \leq n$. Then let

$$
q\left(\sigma\left(x_{1}, \ldots, x_{m}\right)\right) \rightarrow \sigma^{q}\left(q_{j_{1}}\left(x_{i_{j_{1}}}\right), \ldots, q_{j_{n}}\left(x_{i_{j_{n}}}\right)\right)
$$

be in $R^{\prime}$ and let

$$
p\left(\sigma^{q}\left(x_{1}, \ldots, x_{n}\right)\right) \rightarrow t\left[p\left(x_{k_{1}}\right), \ldots, p\left(x_{k_{n}}\right)\right]
$$

be in $R^{\prime \prime}$.

Additionally, for every reducing rule $q\left(\sigma\left(x_{1}, \ldots, x_{m}\right)\right) \rightarrow q^{\prime}\left(x_{i}\right) \in R$, let that rule be also in $R^{\prime}$.

Clearly, in this case $T^{\prime}$ is op-ni-dt and $T^{\prime \prime}$ is nr-l-nd-hom. Moreover, it is easy to verify that $\tau_{T}=\tau_{T^{\prime}} \circ \tau_{T^{\prime \prime}}$. Hence $D T \subseteq$ op-ni-DT $\circ \mathrm{nr}-\mathrm{l}-\mathrm{dn}-H O M$.

Moreover, we show that the total version of the previous theorem also holds.

Corollary 2.1.10 $t$-DT $=t$-op-ni-DT o $n r-l-n d-H O M$

Proof. It is sufficient to observe that if $T$ in the proof of Lemma 2.1.9 is total, then $T^{\prime}$ will be total as well.

We know (see Proposition 1.4.1) that the syntactic composition of $\mathrm{dt}$ tree transducers preserves any of the properties t, 1 , nd, and hom. We have studied this problem for the sl property and have obtained the following result.

Lemma 2.1.11 The syntactic composition $T^{\prime \prime}=T$ o $T^{\prime}$ of two sl-dt tree transducers $T=\left(Q, \Sigma, \Delta, q_{0}, R\right)$ and $T^{\prime}=\left(Q^{\prime}, \Delta, \Omega, q_{0}^{\prime}, R^{\prime}\right)$ is an sl-dt tree transducer if and only if $T$ is nonreducing or $Q^{\prime}$ is a singleton set.

Proof. We recall that $T^{\prime \prime}=\left(Q^{\prime} \times Q, \Sigma, \Omega,\left(q_{0}^{\prime}, q_{0}\right), R^{\prime \prime}\right)$, where $R^{\prime \prime}$ is defined in the way described in Subsection 2.5. Moreover, by (3) of Proposition 1.4.1, $T^{\prime \prime}$ is linear.

First suppose that $T$ does not have the nonreducing property and $Q^{\prime}$ is not a singleton set. This means that there is a reducing rule $q\left(\sigma\left(x_{1}, \ldots, x_{m}\right)\right) \rightarrow q_{1}\left(x_{i}\right)$ in $R$ and there are two different states $p$ and $p^{\prime}$ in $Q^{\prime}$. In this case, by the definition of $R^{\prime \prime}$, both the rules

$$
(p, q)\left(\sigma\left(x_{1}, \ldots, x_{m}\right)\right) \rightarrow\left(p, q_{1}\right)\left(x_{i}\right)
$$


and

$$
\left(p^{\prime}, q\right)\left(\sigma\left(x_{1}, \ldots, x_{m}\right)\right) \rightarrow\left(p^{\prime}, q_{1}\right)\left(x_{i}\right)
$$

are in $R^{\prime \prime}$. Then $x_{i}$ appears both in $\operatorname{rhs}((p, q), \sigma)$ and $\operatorname{rhs}\left(\left(p^{\prime}, q\right), \sigma\right)$, consequently $T^{\prime \prime}$ is not an sl-dt transducer.

Next, suppose that $Q^{\prime}=\{p\}$ is a singleton set. Then, for every rule

$$
q\left(\sigma\left(x_{1}, \ldots, x_{m}\right)\right) \rightarrow t\left[q_{1}\left(x_{i_{1}}\right), \ldots, q_{n}\left(x_{i_{n}}\right)\right] \in R,
$$

at most one rule of the form

$$
(p, q)\left(\sigma\left(x_{1}, \ldots, x_{m}\right)\right) \rightarrow t^{\prime}\left[\left(p, q_{j_{1}}\right)\left(x_{i_{j_{1}}}\right), \ldots,\left(p, q_{j_{k}}\right)\left(x_{i_{j_{k}}}\right)\right]
$$

can be obtained for $R^{\prime}$, where the right-hand side is determined by the conditions

$$
p(t) \underset{T^{\prime}}{\stackrel{*}{\Rightarrow}} t^{\prime}\left[p\left(x_{j_{1}}\right), \ldots, p\left(x_{j_{k}}\right)\right]
$$

and $t^{\prime} \in \hat{T}_{\Delta, k}$. Moreover, by inspection, $\operatorname{var}(\operatorname{rhs}((p, q), \sigma)) \subseteq \operatorname{var}(\operatorname{rhs}(q, \sigma))$. Consequently, if $q$ and $q^{\prime}$ are different states in $Q$, then

$$
\operatorname{var}(\operatorname{rhs}((p, q), \sigma)) \cap \operatorname{var}\left(\operatorname{rhs}\left(\left(p, q^{\prime}\right), \sigma\right)\right) \subseteq \operatorname{var}(\operatorname{rhs}(q, \sigma)) \cap \operatorname{var}\left(\operatorname{rhs}\left(q^{\prime}, \sigma\right)\right)=\emptyset,
$$

showing that $T^{\prime \prime}$ is superlinear.

Finally, assume that $T$ is an nr-sl-dt tree transducer. We must show that

$$
\operatorname{var}(\operatorname{rhs}((p, q), \sigma)) \cap \operatorname{var}\left(\operatorname{rhs}\left(\left(p^{\prime}, q^{\prime}\right), \sigma\right)\right)=\emptyset,
$$

for all states $p, p^{\prime} \in Q^{\prime}$ and $q, q^{\prime} \in Q$ such that $p \neq p^{\prime}$ or $q \neq q^{\prime}$.

In the case when $q \neq q^{\prime}$ our statement follows from

$$
\operatorname{var}(\operatorname{rhs}(q, \sigma)) \cap \operatorname{var}\left(\operatorname{rhs}\left(q^{\prime}, \sigma\right)\right)=\emptyset
$$

by an argumentation similar to the previous one.

Thus, the only case we have to deal with is $q=q^{\prime}$ and $p \neq p^{\prime}$. Therefore, take a rule

$$
q\left(\sigma\left(x_{1}, \ldots, x_{m}\right)\right) \rightarrow t\left[q_{1}\left(x_{i_{1}}\right), \ldots, q_{n}\left(x_{i_{n}}\right)\right] \in R,
$$

where $t \neq x_{1}$ holds by the nonreducing property. Moreover, suppose that

$$
p(t) \underset{T^{\prime}}{\stackrel{*}{\Rightarrow}} t^{\prime}\left[p_{1}\left(x_{j_{1}}\right), \ldots, p_{k}\left(x_{j_{k}}\right)\right]
$$

and

$$
p^{\prime}(t) \underset{T^{\prime}}{\stackrel{*}{\Rightarrow}} t^{\prime \prime}\left[p_{1}^{\prime}\left(x_{j_{1}^{\prime}}\right), \ldots, p_{l}^{\prime}\left(x_{j_{l}^{\prime}}\right)\right]
$$

In this case both the rules

$$
(p, q)\left(\sigma\left(x_{1}, \ldots, x_{m}\right)\right) \rightarrow t^{\prime}\left[\left(p_{1}, q_{j_{1}}\right)\left(x_{i_{j_{1}}}\right), \ldots,\left(p_{k}, q_{j_{k}}\right)\left(x_{i_{j_{k}}}\right)\right]
$$


and

$$
\left(p^{\prime}, q\right)\left(\sigma\left(x_{1}, \ldots, x_{m}\right)\right) \rightarrow t^{\prime \prime}\left[\left(p_{1}^{\prime}, q_{j_{1}^{\prime}}\right)\left(x_{i_{j_{1}^{\prime}}}\right), \ldots,\left(p_{l}^{\prime}, q_{j_{l}^{\prime}}\right)\left(x_{i_{j_{l}^{\prime}}}\right)\right]
$$

are in $R^{\prime \prime}$. Since $T^{\prime}$ is superlinear, we have $\left\{j_{1}, \ldots, j_{k}\right\} \cap\left\{j_{1}^{\prime}, \ldots, j_{l}^{\prime}\right\}=\emptyset$, by Lemma 2.1.8.

On the other hand, since $T$ is linear as well, the integers $i_{1}, \ldots, i_{n}$ in $(*)$ are pairwise different. Hence we have also $\left\{i_{j_{1}}, \ldots, i_{j_{k}}\right\} \cap\left\{i_{j_{1}^{\prime}}, \ldots, i_{j_{l}^{\prime}}\right\}=\emptyset$, which means that $\operatorname{var}(\operatorname{rhs}((p, q), \sigma)) \cap \operatorname{var}\left(\operatorname{rhs}\left(\left(p^{\prime}, q\right), \sigma\right)\right)=\emptyset$. Thus $T^{\prime \prime}$ is superlinear in this case, too.

Many equations regarding sl-DT can be derived from the results of Lemma 2.1.9, Corollary 2.1.10, and Lemma 2.1.11. We list that ones, which will be used in what follows.

\section{Corollary 2.1.12}
(1) $t-s l-D T$
$=t-s l-D T \circ l-H O M$
(2) $s l-D T$
$=s l-D T \circ l-n d-H O M$
(3) $s l-D T$
$=t-n r-s l-D T \circ s l-D T$
(4) $s l-D T$
$=$ op-ni-sl-DT o nr-l-nd-HOM
(5) $t-s l-D T$
$=t$-op-ni-sl-DT o nr-l-nd-HOM
(6) $s l-D T$
$=n r-l-n d-H O M \circ s l-D T$
(7) op-ni-sl-DT
$=\quad t-n r-o p-n i-s l-D T \circ o p-n i-s l-D T$

\section{Proof.}

(1) Since $I \subseteq l-H O M$, the inclusion $t$-sl-DT $\subseteq t$-sl-DT $0 l$-HOM should be obvious. To show its converse, consider a t-sl-dt tree transducer $T^{\prime}$, an 1-hom tree transducer $T^{\prime \prime}$ and the tree transducer $T=T^{\prime} \circ T^{\prime \prime}$. Since $T^{\prime}$ and $T^{\prime \prime}$ are total, $T$ is total as well, by (3) of Proposition 1.4.1. Moreover, $\tau_{T}=\tau_{T^{\prime}} \circ \tau_{T^{\prime \prime}}$ holds, by (1) of the same proposition. Both $T^{\prime}$ and $T^{\prime \prime}$ are superlinear (see Observation 2.1.1) and, being a hom tree transducer, $T^{\prime \prime}$ has a singleton state set. Hence $T$ is also superlinear by Lemma 2.1.11. With this we proved that the inclusion t-sl-DT o $l-H O M \subseteq t$-sl-DT also holds.

(2) The inclusion $s l-D T \subseteq s l-D T \circ l-n d-H O M$ obviously holds. Conversely, let $T^{\prime}$ be an sl-dt tree transducer, let $T^{\prime \prime}$ be an nd-l-hom tree transducer and put $T=T^{\prime} \circ T^{\prime \prime}$. Then, by Lemma 2.1.11, $T$ is superlinear. Moreover, by (1) of Proposition 1.4.1, $\tau_{T}=\tau_{T^{\prime}} \circ \tau_{T^{\prime \prime}}$ holds. Hence the reversed inclusion.

(3) The inclusion sl-DT $\subseteq t$-nr-sl-DT o sl-DT should be clear. Let $T^{\prime}$ be a t-nr-sl-dt tree transducer, let $T^{\prime \prime}$ be an sl-dt tree transducer and let $T=T^{\prime} \circ T^{\prime \prime}$. Then $T$ is superlinear by Lemma 2.1.11. Moreover, $\tau_{T}=\tau_{T^{\prime}} \circ \tau_{T^{\prime \prime}}$ holds, by (1) of Proposition 1.4.1. Hence the reversed inclusion.

(4) The inclusion op-ni-sl-DT onr-l-nd-HOM $\subseteq s l-D T$ holds by (5) of Corollary 1.4.2 and by Lemma 2.1.11. Conversely, let $T$ be an sl-dt tree transducer. Let the op-ni-dt tree transducer $T^{\prime}$ and the nr-l-nd-hom tree transducer $T^{\prime \prime}$ be 
constructed from $T$ in the same way as in the proof of Lemma 2.1.9. Observe, that $T^{\prime}$ will be superlinear, by that construction. Hence $s l-D T \subseteq$ op-ni-sl-DT $\circ \mathrm{nr}-\mathrm{l}$ $n d-H O M$ holds.

(5) This should be clear by (4) and by Corollary 2.1.10.

(6) It holds that $I \subseteq n r-l-n d-H O M$, hence $s l-D T \subseteq n r-l-n d-H O M \circ s l-D T$. Moreover, since $n r-l-n d-H O M \subseteq s l-D T$ (see Corollary 2.1.2), the inclusion $n r-l-n d-H O M \circ s l-D T \subseteq s l-D T$ is obvious by Lemma 2.1.11 and Proposition 1.4.1.

(7) Letting $C=t$-nr-op-ni-sl-DT o op-ni-sl-DT, we have op-ni-sl-DT $\subseteq C$ by $I \subseteq t$-nr-op-ni-sl-DT. Moreover, $C \subseteq s l-D T$ holds by Lemma 2.1.11. On the other hand, by the proof of that lemma, it is easy to see that $C \subseteq$ op-ni-sl-DT holds as well.

Theorem 2.1.4 shows that there are two sl-dt tree transformations such that their composition cannot be induced by a dt and hence by an 1-dt tree transducer. This suggests that the consecutive application of a sequence of sl-dt tree transducers has big transformation power, that is, for instance, any l-dt or dt tree transformation can be represented as composition of sl-dt tree transformations.

However, the following theorem shows that this is not the case. Namely, we show that generally even the total l-dt tree transformations cannot be induced by sequences of sl-dt tree transformations.

Theorem 2.1.13 $t-l-D T-s l-D T^{+} \neq \emptyset$

Proof. Let the t-l-dt tree transducer $T=(Q, \Sigma, \Delta, q, R)$ be defined as follows:

- $Q=\left\{q, q^{\prime}\right\}$.

- $\Sigma=\left\{\sigma^{(1)}, \#^{(0)}\right\}$.

- $\Delta=\Sigma \cup\left\{\$^{(0)}\right\}$.

- $R=\left\{q\left(\sigma\left(x_{1}\right)\right) \rightarrow \sigma\left(q^{\prime}\left(x_{1}\right)\right), q^{\prime}\left(\sigma\left(x_{1}\right)\right) \rightarrow \sigma\left(q\left(x_{1}\right)\right), q(\#) \rightarrow \#, q^{\prime}(\#) \rightarrow \$\right\}$.

It should be clear that

$$
\tau_{T}=\left\{\left(\sigma^{m}(\#), \sigma^{m}(\#)\right) \mid m \geq 0 \text { is even }\right\} \cup\left\{\left(\sigma^{m}(\#), \sigma^{m}(\$)\right) \mid m \geq 0 \text { is odd }\right\} .
$$

We prove by contradiction that $\tau_{T} \notin s l-D T^{n}$ for any $n \geq 1$.

Therefore, suppose that an integer $n \geq 1$ and sl-dt tree transducers $T_{1}, \ldots, T_{n}$ exist such that $\tau_{T}=\tau_{T_{1}} \circ \cdots \circ \tau_{T_{n}}$ holds. Without loss of generality, we can assume that $n$ is chosen to be minimal. We put $\tau_{n}=\tau_{T_{1}} \circ \cdots \circ \tau_{T_{n}}$.

Let $T_{1}=\left(Q_{1}, \Sigma, \Sigma^{\prime}, q_{1}, R_{1}\right)$. Observe that, since $(\#, \#) \in \tau_{T}$, there must be a $\left(q_{1}, \#\right)$-rule in $R_{1}$ of the form

$$
q_{1}(\#) \rightarrow s
$$


where $s \in T_{\Sigma^{\prime}}$.

Similarly, $\tau_{T}(\sigma(\#))=\sigma(\$)$ implies that there must be a $\left(q_{1}, \sigma\right)$-rule in $R_{1}$. Moreover, $\operatorname{rhs}\left(q_{1}, \sigma\right)$ must contain the variable $x_{1}$. Otherwise, $\operatorname{rhs}\left(q_{1}, \sigma\right) \in T_{\Sigma^{\prime}}$ implies $\tau_{T_{1}}(\sigma(\#))=\tau_{T_{1}}\left(\sigma^{2}(\#)\right)$ and hence $\tau_{n}(\sigma(\#))=\tau_{n}\left(\sigma^{2}(\#)\right)$, which contra$\operatorname{dicts} \tau_{T}\left(\sigma^{2}(\#)\right)=\sigma^{2}(\#)$.

Since $T_{1}$ is linear, the $\left(q_{1}, \sigma\right)$-rule is of the form

$$
q_{1}\left(\sigma\left(x_{1}\right)\right) \rightarrow r\left[p\left(x_{1}\right)\right],
$$

for some $r \in \hat{T}_{\Sigma^{\prime}, 1}$ and $p \in Q_{1}$.

Since $\tau_{T}\left(\sigma^{2}(\#)\right)=\sigma^{2}(\#)$, there must be a $(p, \sigma)$-rule in $R_{1}$, too. Assume that $p \neq q_{1}$. Then $\operatorname{rhs}(p, \sigma)$ cannot contain $x_{1}$, because $\operatorname{rhs}\left(q_{1}, \sigma\right)$ already does so and $T_{1}$ is superlinear. However, this implies $\tau_{T_{1}}\left(\sigma^{2}(\#)\right)=\tau_{T_{1}}\left(\sigma^{3}(\#)\right)$, which contradicts $\tau_{T}\left(\sigma^{3}(\#)\right)=\sigma^{3}(\$)$. Therefore, $p=q_{1}$ holds and thus the rule

$$
q_{1}\left(\sigma\left(x_{1}\right)\right) \rightarrow r\left[q_{1}\left(x_{1}\right)\right]
$$

is in $R_{1}$.

We can observe that only the two $q_{1}$-rules

$$
q_{1}(\#) \rightarrow s
$$

and

$$
q_{1}\left(\sigma\left(x_{1}\right)\right) \rightarrow r\left[q_{1}\left(x_{1}\right)\right]
$$

can be useful in any derivation using $T_{1}$, where $s \in T_{\Sigma^{\prime}}$ and $r \in \hat{T}_{\Sigma^{\prime}, 1}$. Hence $T_{1}$ is total.

Next we show that the tree $r \in \hat{T}_{\Sigma, 1}$ appearing in the above $\left(q_{1}, \sigma\right)$-rule cannot be $x_{1}$, that is the rule cannot be a reducing one.

For if $r=x_{1}$, i.e. $q_{1}\left(\sigma\left(x_{1}\right)\right) \rightarrow q_{1}\left(x_{1}\right)$ is in $R_{1}$, then $q_{1}\left(\sigma^{m}(\#)\right) \Rightarrow_{T_{1}}^{*} s$ holds, for every $m \geq 0$, implying $\left|\operatorname{range}\left(\tau_{n}\right)\right|=1$, which is obviously not true. Hence $T_{1}$ is nonreducing and thus it is a t-nr-sl-dt tree transducer.

By (3) of Corollary 2.1.12 and by (1) of Proposition 1.4.1, the tree transducer $T_{2}^{\prime}=T_{1} \circ T_{2}$ is superlinear and $\tau_{T_{2}^{\prime}}=\tau_{T_{1}} \circ \tau_{T_{2}}$ holds. Hence we have $\tau_{T}=$ $\tau_{T_{2}^{\prime}} \circ \tau_{T_{3}} \circ \ldots \circ \tau_{T_{n}}$, which contradicts our assumption that $n$ is the smallest integer such that $\tau_{T}$ is a composition of $n$ sl-dt tree transformations.

Considering Theorem 2.1.13, we can easily show the following inclusions, which proves to be very useful in later chapters.

\section{Corollary 2.1.14}

$$
\begin{array}{lll}
\text { (1) } s l-D T^{n} & \subset l-D T^{n}, \text { for every } n \geq 1 \text {. } \\
\text { (2) } s l-D T^{+} & \subset l-D T^{2} \text {. } \\
\text { (3) } t \text {-sl-DT } & \subset t-l-D T .
\end{array}
$$


Proof. We recall $l-D T \subset l-D T^{2}$ (see [Rou]) and $l-D T^{n}=l-D T^{2}$, for $n \geq 2$, see Proposition 1.4.3.

(1) Clearly, sl-DT $\subseteq l-D T^{n}$. On the other hand, sl-DT $=l-D T^{n}$, for some $n \geq 1$, would imply $t-l-D T \subset s l-D T^{n}$, which contradicts Theorem 2.1.13.

(2) We have sl-DT $T^{+} \subseteq l-D T^{+}=l-D T^{2}$. Clearly, sl-DT $T^{+}=l-D T^{2}$ implies $t-l-D T \subset s l-D T^{+}$contradicting again Theorem 2.1.13.

(3) By Proposition 1.4.1, it is easy to see that $t-l-D T^{+}=t-l-D T$, hence $t$-st-DT $\subseteq t-l-D T$ holds. Moreover, the proper inclusion immediately follows from Theorem 2.1.13.

\subsection{Domain tree languages}

In this section we give a characterization of the class dom(sl-DT). Moreover, we show that, for any $L \in D R E C$, it is decidable whether $L \in \operatorname{dom}(s l-D T)$ holds and we present also a decision procedure.

Let $T=\left(Q, \Sigma, \Sigma, q_{0}, R\right)$ be a dttr. We say that $T$ is a semi-universal deterministic top-down tree recognizer (su-dttr), if the following condition holds. For any $m \geq 1, \sigma \in \Sigma_{m}$, and two different states $q, p \in Q$, if $q\left(\sigma\left(x_{1}, \ldots, x_{m}\right)\right) \rightarrow$ $\sigma\left(q_{1}\left(x_{1}\right), \ldots, q_{m}\left(x_{m}\right)\right)$ and $p\left(\sigma\left(x_{1}, \ldots, x_{m}\right)\right) \rightarrow \sigma\left(p_{1}\left(x_{1}\right), \ldots, p_{m}\left(x_{m}\right)\right)$ are in $R$, then, for each $1 \leq i \leq m$, at least one of $q_{i}$ and $p_{i}$ is universal. We denote by su-DREC the class of tree languages recognized by su-dttr's.

First we show that $\operatorname{dom}(s l-D T)$ and $s u-D R E C$ are equal classes.

Lemma 2.2.1 For any sl-dt tree transducer $T=\left(Q, \Sigma, \Delta, q_{0}, R\right), \operatorname{dom}\left(\tau_{T}\right)$ is recognized by an su-dttr.

Proof. Let the dttr $T^{\prime}=\left(Q^{\prime}, \Sigma, \Sigma,\left\{q_{0}\right\}, R^{\prime}\right)$ be constructed from $T$ as defined in the proof of Proposition 1.4.11, then $L\left(T^{\prime}\right)=\operatorname{dom}\left(\tau_{T}\right)$. We show that $T^{\prime}$ is an su-dttr.

Since $T$ is linear, each set in $Q^{\prime}$ contains at most one element. Observe that, for any $m \geq 1, \sigma \in \Sigma_{m}$, and $q \in Q$, if $\{q\}\left(\sigma\left(x_{1}, \ldots, x_{m}\right)\right) \rightarrow \sigma\left(P_{1}\left(x_{1}\right), \ldots, P_{m}\left(x_{m}\right)\right)$ is in $R^{\prime}$, then $q\left(\sigma\left(x_{1}, \ldots, x_{m}\right)\right) \rightarrow t\left[q_{1}\left(x_{i_{1}}\right), \ldots, q_{n}\left(x_{i_{n}}\right)\right] \in R$, for some $0 \leq n \leq m$ and $t \in \hat{T}_{\Delta, n}$. Moreover, for each $1 \leq j \leq m$, if $j=i_{k}$, for some $1 \leq k \leq n$, then $P_{j}=\left\{q_{k}\right\}$, and $P_{j}=\emptyset$ otherwise. Note that, since $T$ is linear, the $i_{k}$ s are different.

Now suppose that, for some $m \geq 1, \sigma \in \Sigma_{m}$ and two different states $q, p \in$ $Q,\{q\}\left(\sigma\left(x_{1}, \ldots, x_{m}\right)\right) \rightarrow \sigma\left(P_{q, 1}\left(x_{1}\right), \ldots, P_{q, m}\left(x_{m}\right)\right)$ and $\{p\}\left(\sigma\left(x_{1}, \ldots, x_{m}\right)\right) \rightarrow$ $\sigma\left(P_{p, 1}\left(x_{1}\right), \ldots, P_{p, m}\left(x_{m}\right)\right)$ are in $R^{\prime}$. Then, by the above observations, there exist rules $q\left(\sigma\left(x_{1}, \ldots, x_{m}\right)\right) \rightarrow t\left[q_{1}\left(x_{i_{1}}\right), \ldots, q_{n}\left(x_{i_{n}}\right)\right]$ and $p\left(\sigma\left(x_{1}, \ldots, x_{m}\right)\right) \rightarrow$ $s\left[p_{1}\left(x_{i_{1}^{\prime}}\right), \ldots, p_{n^{\prime}}\left(x_{i_{n^{\prime}}}\right)\right]$ in $R$, for some $0 \leq n, n^{\prime} \leq m, t \in \hat{T}_{\Delta, n}$, and $s \in \hat{T}_{\Delta, n^{\prime}}$, where, for each $1 \leq j \leq m$, if $j=i_{k}$, for some $1 \leq k \leq n$, then $P_{q, j}=\left\{q_{k}\right\}$, else 
$P_{q, j}=\emptyset$. Moreover, if $j=i_{k}^{\prime}$, for some $1 \leq k \leq n^{\prime}$, then $P_{p, j}=\left\{p_{k}\right\}$, and $P_{p, j}=\emptyset$ otherwise.

Since $T$ is superlinear, $\left\{i_{1}, \ldots, i_{n}\right\} \cap\left\{i_{1}^{\prime}, \ldots, i_{n^{\prime}}^{\prime}\right\}=\emptyset$, hence we have that, for each $1 \leq j \leq m$, at least one of $P_{q, j}$ and $P_{p, j}$ is $\emptyset$. We saw that if $\emptyset \in Q^{\prime}$, then it is necessarily a universal state, hence $T^{\prime}$ is an su-dttr.

Lemma 2.2.2 For any $L \in$ su-DREC, an sl-dt tree transducer $T^{\prime}$ exists such that $\operatorname{dom}\left(\tau_{T^{\prime}}\right)=L$.

Proof. Suppose that $L \in s u-D R E C$, then it is recognized by an su-dttr $T=\left(Q, \Sigma, \Sigma, q_{0}, R\right)$. For every $m \geq 0, \sigma \in \Sigma_{m}, q \in Q$, and $q\left(\sigma\left(x_{1}, \ldots, x_{m}\right)\right) \rightarrow$ $\sigma\left(q_{1}\left(x_{1}\right), \ldots, q_{m}\left(x_{m}\right)\right) \in R$, consider the set $\left\{i_{1}, \ldots, i_{n}\right\} \subseteq\{1, \ldots, m\}$ of indices, where $i_{1}<\ldots<i_{n}$ and, for any $1 \leq j \leq m, j \in\left\{i_{1}, \ldots, i_{n}\right\}$ holds if and only if $q_{j}$ is not a universal state. Then let $\sigma_{q}$ be a new symbol having the rank $n$ and define the rule $r_{q, \sigma}: q\left(\sigma\left(x_{1}, \ldots, x_{m}\right)\right) \rightarrow \sigma_{q}\left(q_{i_{1}}\left(x_{i_{1}}\right), \ldots, q_{i_{n}}\left(x_{i_{n}}\right)\right)$.

Let $T^{\prime}=\left(Q, \Sigma, \Delta, q_{0}, R^{\prime}\right)$ be a dt transducer, where

- $\Delta=\left\{\sigma_{q} \mid q\right.$ is defined on $\sigma$ in $\left.R\right\}$ and

- $R^{\prime}=\left\{r_{q, \sigma} \mid q\right.$ is defined on $\sigma$ in $\left.R\right\}$.

We show that $T^{\prime}$ is superlinear. Obviously, it is linear. Let $q, p \in Q$ be two different states. Suppose that $q\left(\sigma\left(x_{1}, \ldots, x_{m}\right)\right) \rightarrow \sigma_{q}\left(q_{1}\left(x_{i_{1}}\right), \ldots, q_{k}\left(x_{i_{k}}\right)\right)$ and $p\left(\sigma\left(x_{1}, \ldots, x_{m}\right)\right) \rightarrow \sigma_{p}\left(p_{1}\left(x_{j_{1}}\right), \ldots, p_{l}\left(x_{j_{l}}\right)\right)$ are in $R$, for some $m \geq 0,0 \leq k, l \leq m$ and $\sigma \in \Sigma_{m}$. Then, by the construction of $T^{\prime},\left\{q_{i_{1}}, \ldots, q_{i_{k}}\right\}$ and $\left\{q_{j_{1}}, \ldots, q_{j_{l}}\right\}$ are the sets of non-universal states of $\operatorname{rhs}(q, \sigma)$ and $\operatorname{rhs}(p, \sigma)$ in $T$, respectively. Since $T$ is an su-dttr, $\left\{i_{1}, \ldots, i_{k}\right\} \cap\left\{j_{1}, \ldots, j_{l}\right\}=\emptyset$ holds. Therefore $T^{\prime}$ is superlinear.

Finally, we show that, for any tree $t \in T_{\Sigma}$ and state $q \in Q, q(t) \Rightarrow_{T}^{*} t$ holds if and only if $q(t) \Rightarrow_{T^{\prime}}^{*} t^{\prime}$, for some $t^{\prime} \in T_{\Delta}$. This implies $\operatorname{dom}\left(\tau_{T}\right)=L$ immediately. We prove the statement by induction on height $(t)$.

Basis. Suppose that height $(t)=0$, then $t=\delta$, for some $\delta \in \Sigma_{0}$. By the definition of $T^{\prime}, q(\delta) \rightarrow \delta_{q} \in R^{\prime}$ if and only if $q(\delta) \rightarrow \delta \in R$, hence the statement holds by $t^{\prime}=\delta_{q}$.

Induction step. Suppose that height $(t)=n+1$ with $n \geq 0$, then $t=$ $\sigma\left(t_{1}, \ldots, t_{m}\right)$, for some $m \geq 1, \sigma \in \Sigma_{m}$, and $t_{1}, \ldots, t_{m} \in T_{\Sigma}$, where height $\left(t_{i}\right) \leq n$, for each $1 \leq i \leq m$.

Recall that $q\left(\sigma\left(x_{1}, \ldots, x_{m}\right)\right) \rightarrow \sigma_{q}\left(q_{i_{1}}\left(x_{i_{1}}\right), \ldots, q_{i_{n}}\left(x_{i_{n}}\right)\right) \in R^{\prime}$ if and only if $q\left(\sigma\left(x_{1}, \ldots, x_{m}\right)\right) \rightarrow \sigma\left(q_{1}\left(x_{1}\right), \ldots, q_{m}\left(x_{m}\right)\right) \in R$, where $q_{i_{1}}, \ldots, q_{i_{n}}$ are exactly the non-universal states of $\operatorname{rhs}(q, \sigma)$ in $T$. Furthermore, by the induction hypothesis, for each $j \in\left\{i_{1}, \ldots, i_{n}\right\}, q_{j}\left(t_{j}\right) \Rightarrow_{T}^{*} t_{j}$ holds if and only if $q_{j}\left(t_{j}\right) \Rightarrow_{T^{\prime}}^{*} t_{j}^{\prime}$, for some $t_{j}^{\prime} \in T_{\Delta}$. Hence $q(t) \Rightarrow_{T} \sigma\left(q_{1}\left(t_{1}\right), \ldots, q_{m}\left(t_{m}\right)\right) \Rightarrow_{T}^{*} t$ if and only if $q(t) \Rightarrow_{T^{\prime}}$ $\sigma_{q}\left(q_{i_{1}}\left(t_{i_{1}}\right), \ldots, q_{i_{n}}\left(t_{i_{n}}\right)\right) \Rightarrow T_{T^{\prime}}^{*} t^{\prime}$, where $t^{\prime}=\sigma_{q}\left(t_{i_{1}}^{\prime}, \ldots, t_{i_{n}}^{\prime}\right)$. 
Summarizing the results of the above two lemmas, we have that the domain tree languages of sl-dt tree transformations are exactly those tree languages, which are recognized by su-dttr's.

Theorem 2.2.3 dom $(s l-D T)=s u-D R E C$

In the rest of the section we show that, for any $L \in D R E C$ given by a dttr recognizing $L$, it is decidable whether $L \in \operatorname{dom}(s l-D T)$ holds. Moreover, we present a decision procedure.

Recall that, for a dttr $T, T_{\text {nor }}$ and $T_{\text {con }}$ denote the normalized and connected equivalents of $T$, according to Propositions 1.4 .7 and 1.4.8, respectively. Moreover, if $T$ is normalized and connected, then $T_{\min }$ is the minimal equivalent of $T$, according to Proposition 1.4.9.

Lemma 2.2.4 Let $T=\left(Q, \Sigma, \Sigma, q_{0}, R\right)$ be an su-dttr, then $T_{\text {nor }}$ and $T_{\text {con }}$ are su-dttr's, too. Moreover, if $T$ is normalized and connected, then $T_{\min }$ is also an su-dttr.

Proof. By Proposition 1.4.7, $T_{\text {nor }}=\left(Q^{\prime}, \Sigma, \Sigma, q_{0}, R^{\prime}\right)$, where $Q^{\prime} \subseteq Q$ and $R^{\prime} \subseteq R$ hold. Hence it should be clear that if $T$ is an su-dttr, then $T_{n o r}$ is also an su-dttr.

Similarly, by Proposition 1.4.8, $T_{\text {con }}=\left(Q^{\prime \prime}, \Sigma, \Sigma, q_{0}, R^{\prime \prime}\right)$, where $Q^{\prime \prime} \subseteq Q$ and $R^{\prime \prime} \subseteq R$. Therefore if $T$ is an su-dttr, then $T_{\text {con }}$ is necessarily an su-dttr, too.

Now suppose that $T$ is a normalized and connected su-dttr. Denote by $\equiv$ the equivalence relation, by which $T_{\min }$ is constructed from $T$ (see Construction 1.4.10), that is $T_{\min }=\left(Q^{\prime \prime \prime}, \Sigma, \Sigma,\left[q_{0}\right]_{\equiv}, R^{\prime \prime \prime}\right)$, where $R^{\prime \prime \prime}=\left\{[q]_{\equiv}\left(\sigma\left(x_{1}, \ldots, x_{m}\right)\right) \rightarrow\right.$ $\left.\sigma\left(\left[q_{1}\right]_{\equiv}\left(x_{1}\right), \ldots,\left[q_{m}\right]_{\equiv}\left(x_{m}\right)\right) \mid q\left(\sigma\left(x_{1}, \ldots, x_{m}\right)\right) \rightarrow \sigma\left(q_{1}\left(x_{1}\right), \ldots, q_{m}\left(x_{m}\right)\right) \in R\right\}$ and $Q^{\prime \prime \prime}=\left\{[q]_{\equiv} \mid q \in Q\right\}$.

It can be easily shown that if $T$ has universal states, then they constitute exactly one class of $Q$ with respect to $\equiv$. Moreover, if this class exists, then it is the only universal state in $T_{\text {min }}$. By the construction of $\equiv$, the proofs of these statements are straightforward.

Suppose that the states $q, p \in Q$ are in different classes with respect to $\equiv$, that is $[q]_{\equiv} \neq[p]_{\equiv}$. If, for some $\sigma \in \Sigma_{m}$ with $m \geq 1$, both $[q]_{\equiv}$ and $[p]_{\equiv}$ are defined on $\sigma$ in $R^{\prime \prime \prime}$, then the $\left([q]_{\equiv}, \sigma\right)$-rule and the $\left([p]_{\equiv}, \sigma\right)$-rule of $R^{\prime \prime \prime}$ can be written of the form $[q]_{\equiv}\left(\sigma\left(x_{1}, \ldots, x_{m}\right)\right) \rightarrow \sigma\left(\left[q_{1}\right]_{\equiv}\left(x_{1}\right), \ldots,\left[q_{m}\right]_{\equiv}\left(x_{m}\right)\right)$ and $[p]_{\equiv}\left(\sigma\left(x_{1}, \ldots, x_{m}\right)\right) \rightarrow \sigma\left(\left[p_{1}\right]_{\equiv}\left(x_{1}\right), \ldots,\left[p_{m}\right]_{\equiv}\left(x_{m}\right)\right)$, respectively, where $q_{1}, \ldots, q_{m}$, $p_{1}, \ldots, p_{m} \in Q$ and the rules $q\left(\sigma\left(x_{1}, \ldots, x_{m}\right)\right) \rightarrow \sigma\left(q_{1}\left(x_{1}\right), \ldots, q_{m}\left(x_{m}\right)\right)$ and $p\left(\sigma\left(x_{1}, \ldots, x_{m}\right)\right) \rightarrow \sigma\left(p_{1}\left(x_{1}\right), \ldots, p_{m}\left(x_{m}\right)\right)$ are in $R$. Since $T$ is an su-dttr, then, for any $1 \leq i \leq m$, at least one of $q_{i}$ and $p_{i}$ is universal in $T$. Thus, by the observations of the previous paragraph, at least one of $\left[q_{i}\right]_{\equiv}$ and $\left[p_{i}\right]_{\equiv}$ is universal in $T_{\min }$. Therefore $T_{\min }$ is an su-dttr, too. 
We recall from Proposition 1.4.9 that, for any $L \in D R E C$, the minimal dttr recognizing $L$ is unique up to isomorphism. Denote this $\mathrm{dttr}$ by $T_{L}$. The following theorem establishes our decidability result.

Lemma 2.2.5 For any tree language $L \in D R E C, L \in$ su-DREC if and only if $T_{L}$ is an su-dttr.

Proof. If $T_{L}$ is an su-dttr, then $L \in s u-D R E C$ by definition. Conversely, suppose $L \in$ su-DREC, then an su-dttr $T$ exists such that $L(T)=L$.

By Propositions 1.4.7, 1.4.8 and 1.4.9, $T_{L}$ can be computed from $T$ and, by Lemma 2.2.4, it is an su-dttr, too.

Theorem 2.2.6 For any tree language $L \in D R E C$ given by a dttr $T$ recognizing $L$, it is decidable whether $L \in \operatorname{dom}($ sl-DT) holds.

Proof. By Propositions 1.4.7, 1.4.8 and 1.4.9, $T_{L}$ can be constructed effectively from $T$.

Moreover, it is obviously decidable whether $T_{L}$ is an su-dttr. Hence, by Lemma 2.2.5 and Theorem 2.2.3, the statement of the theorem holds.

Finally, we present an algorithm, which, for any tree language $L \in D R E C$ given by a dttr recognizing $L$, decides whether $L \in \operatorname{dom}(s l-D T)$ holds. The method is based on the proof of the Theorem 2.2.6.

Let $L$ be an arbitrary deterministic recognizable tree language and let $T^{(1)}$ be a dttr, which recognizes $L$.

The algorithm gives the answer YES if $L$ can be the domain of a superlinear deterministic top-down tree transformation, otherwise it answers $N O$.

1. Compute $T_{n o r}^{(1)}$ as defined in the proof of the Proposition 1.4.7. Denote $T_{\text {nor }}^{(1)}$ by $T^{(2)}$.

2. Compute $T_{c o n}^{(2)}$ as defined in the proof of the Proposition 1.4.8. Denote $T_{c o n}^{(2)}$ by $T^{(3)}$.

3. Construct $T_{\text {min }}^{(3)}$ as determined in Construction 1.4.10. Denote $T_{\min }^{(3)}$ by $T_{L}$.

4. Decide whether $T_{L}$ is semi-universal. (It is trivially decidable, e.g., check all rule pairs, which concern the same input symbol.) If it is, then the answer is $Y E S$, else the answer is $N O$. 


\subsection{Range tree languages}

In this section we prove range $(s l-D T)=R E C$. Furthermore, as a by-product, we get range $(l-D T)=R E C$, too.

Assume that $L \in R E C$, then there exists a ttr $T=\left(Q, \Sigma, \Sigma, q_{0}, R\right)$ satisfying $L(T)=L$. We define the ranked alphabet $\Delta$ such that, for each $m \geq 0$, we put

$$
\Delta_{m}=\left\{\sigma_{q, q_{1}, \ldots, q_{m}} \mid \sigma \in \Sigma_{m}, q\left(\sigma\left(x_{1}, \ldots, x_{m}\right)\right) \rightarrow \sigma\left(q_{1}\left(x_{1}\right), \ldots, q_{m}\left(x_{m}\right)\right) \in R\right\} .
$$

Let $T^{\prime}=\left(Q, \Delta, \Sigma, q_{0}, R^{\prime}\right)$ be a dt tree transducer, where

$$
R^{\prime}=\left\{q\left(\sigma_{q, q_{1}, \ldots, q_{m}}\left(x_{1}, \ldots, x_{m}\right)\right) \rightarrow \sigma\left(q_{1}\left(x_{1}\right), \ldots, q_{m}\left(x_{m}\right)\right) \mid \sigma_{q, q_{1}, \ldots, q_{m}} \in \Delta\right\} .
$$

Observe that $T^{\prime}$ is an rl-sl-dt tree transducer.

Lemma 2.3.1 For any tree $t \in T_{\Sigma}$ and state $q \in Q, q(t) \Rightarrow_{T}^{*} t$ if and only if $q\left(t^{\prime}\right) \Rightarrow_{T^{\prime}}^{*} t$ holds, for some $t^{\prime} \in T_{\Delta}$.

Proof. First assume $q(t) \Rightarrow_{T}^{*} t$. We show the existence of the above $t^{\prime}$ by induction on height $(t)$.

Basis. Suppose that height $(t)=0$, then $t=\delta$, for some $\delta \in \Sigma_{0}$, and then $q(t) \Rightarrow_{T}^{*} t$ implies $q(\delta) \rightarrow \delta \in R$. By the construction of $T^{\prime}, \delta_{q} \in \Delta_{0}$ and $q\left(\delta_{q}\right) \rightarrow \delta \in R^{\prime}$, hence $q\left(t^{\prime}\right) \Rightarrow_{T^{\prime}}^{*} t$ holds, for $t^{\prime}=\delta_{q}$.

Induction step. Suppose that height $(t)=n+1$ with $n \geq 0$, then $t=$ $\sigma\left(t_{1}, \ldots, t_{m}\right)$, for some $m \geq 1, \sigma \in \Sigma_{m}$, and $t_{1}, \ldots, t_{m} \in T_{\Sigma}$, where height $\left(t_{i}\right) \leq n$, for all $1 \leq i \leq m$. Since $q(t) \Rightarrow_{T}^{*} t$, there must be a rule $q\left(\sigma\left(x_{1}, \ldots, x_{m}\right)\right) \rightarrow$ $\sigma\left(q_{1}\left(x_{1}\right), \ldots, q_{m}\left(x_{m}\right)\right)$ in $R$, where $q_{i}\left(t_{i}\right) \Rightarrow_{T}^{*} t_{i}$ holds, for each $1 \leq i \leq m$. By the construction of $T^{\prime}, \sigma_{q, q_{1}, \ldots, q_{m}} \in \Delta$ and the rule $q\left(\sigma_{q, q_{1}, \ldots, q_{m}}\left(x_{1}, \ldots, x_{m}\right)\right) \rightarrow$ $\sigma\left(q_{1}\left(x_{1}\right), \ldots, q_{m}\left(x_{m}\right)\right)$ is in $R$. Moreover, by the induction hypothesis, there exist trees $t_{1}^{\prime}, \ldots, t_{m}^{\prime} \in T_{\Delta}$ such that $q_{i}\left(t_{i}^{\prime}\right) \Rightarrow_{T^{\prime}}^{*} t_{i}$, for all $1 \leq i \leq m$. Let $t^{\prime}=\sigma_{q, q_{1}, \ldots, q_{m}}\left(t_{1}^{\prime}, \ldots, t_{m}^{\prime}\right)$, then we have $q\left(t^{\prime}\right) \Rightarrow_{T^{\prime}} \sigma\left(q_{1}\left(t_{1}^{\prime}\right), \ldots, q_{m}\left(t_{m}^{\prime}\right)\right) \Rightarrow_{T^{\prime}}^{*}$ $\sigma\left(t_{1}, \ldots, t_{m}\right)=t$.

Now suppose that there exists a tree $t^{\prime} \in T_{\Delta}$ satisfying $q\left(t^{\prime}\right) \Rightarrow_{T^{\prime}}^{*} t$. We prove $q(t) \Rightarrow_{T}^{*} t$ also by induction on height $(t)$. Recall that, since $T^{\prime}$ is relabeling, height $\left(t^{\prime}\right)=\operatorname{height}(t)$ necessarily holds.

Basis. Suppose that height $(t)=0$. Then $t=\delta$, for some $\delta \in \Sigma_{0}$. By the construction of $T^{\prime}, t^{\prime}=\delta_{q} \in \Delta_{0}$ and $q\left(\delta_{q}\right) \rightarrow \delta \in R^{\prime}$, hence $q(\delta) \rightarrow \delta \in R$. Therefore, $q(t) \Rightarrow_{T}^{*} t$ holds. 
Induction step. Let $n \geq 0$. Suppose that height $(t)=n+1$, then $t=$ $\sigma\left(t_{1}, \ldots, t_{m}\right)$, for some $m \geq 1, \sigma \in \Sigma_{m}$, and $t_{1}, \ldots, t_{m} \in T_{\Sigma}$, where height $\left(t_{i}\right) \leq n$ holds for all $1 \leq i \leq m$. By the construction of $T^{\prime}$, for some $q_{1}, \ldots, q_{m} \in Q$ and $t_{1}^{\prime}, \ldots, t_{m}^{\prime} \in T_{\Delta}, t^{\prime}=\sigma_{q, q_{1}, \ldots, q_{m}}\left(t_{1}^{\prime}, \ldots, t_{m}^{\prime}\right)$ and $q\left(\sigma_{q, q_{1}, \ldots, q_{m}}\left(x_{1}, \ldots, x_{m}\right)\right) \rightarrow$ $\sigma\left(q_{1}\left(x_{1}\right), \ldots, q_{m}\left(x_{m}\right)\right) \in R$ hold. Moreover $q_{i}\left(t_{i}^{\prime}\right) \Rightarrow_{T^{\prime}}^{*} t_{i}$, for each $1 \leq i \leq m$. Hence $q\left(\sigma\left(x_{1}, \ldots, x_{m}\right)\right) \rightarrow \sigma\left(q_{1}\left(x_{1}\right), \ldots, q_{m}\left(x_{m}\right)\right) \in R$ and, by the induction hypothesis, $q_{i}\left(t_{i}\right) \Rightarrow_{T}^{*} t_{i}$, for all $1 \leq i \leq m$. Thercfore, we have $q(t) \Rightarrow_{T}$ $\sigma\left(q_{1}\left(t_{1}\right), \ldots, q_{m}\left(t_{m}\right)\right) \Rightarrow_{T}^{*} \sigma\left(t_{1}, \ldots, t_{m}\right)=t$.

Lemma 2.3.1 implies that, for any tree $t \in T_{\Sigma}, q_{0}(t) \Rightarrow_{T}^{*} t$ holds if and only if there exists a tree $t^{\prime} \in T_{\Delta}$ satisfying $q_{0}\left(t^{\prime}\right) \Rightarrow_{T^{\prime}}^{*} t$. Hence $t \in L(T)$ if and only if $t \in \operatorname{range}\left(\tau_{T^{\prime}}\right)$. Since $L$ was arbitrary and $T^{\prime}$ is rl-sl-dt tree transducer, it follows that $R E C \subseteq \operatorname{range}(s l-D T)$.

On the other hand, range $(s l-D T) \subseteq$ range $(l-D T)$ obviously holds and, by Corollary 6.6 of Chapter IV in [GécSte4], range $(l-D T) \subseteq R E C$, thus we have the following result.

Theorem 2.3.2 range $(s l-D T)=\operatorname{range}(l-D T)=R E C$ 


\section{Chapter 3}

\section{Hierarchy theorems of sl-dt tree transformations}

It turned out in the previous chapter that, similarly to the classes $D T$ and $l-D T$, the class sl-DT is not closed under the composition.

However, in Section 3.1 we show that, in contrast with the classes DT and $l$-DT, the hierarchy $\left\{s l-D T^{n} \mid n \geq 0\right\}$ never collapses. Moreover, we prove in Section 3.2 that even the hierarchy $\left\{t-s l-D T^{n} \mid n \geq 0\right\}$ is proper.

We note that the results of this chapter were published in [DánFül1].

\subsection{The hierarchies $\operatorname{dom}\left(s l-D T^{n}\right)$ and $s l-D T^{n}$}

When a tree transformation class is not closed under the composition, like sl-DT, it is always a fundamental question whether its increasing powers form an infinite hierarchy or not. In other words, whether its power hierarchy collapses at some integer or not.

An example for collapsing hierarchy is $\left\{D T^{n} \mid n \geq 1\right\}$. It was shown in [FülVág1] that $D T^{2}=D T^{3}=\ldots$.

On the other hand, there exists also proper hierarchies. For example, it is shown in [Eng1] that the hierarchy $\left\{N T^{n} \mid n \geq 1\right\}$ is proper, where $N T$ denotes the class of nondeterministic top-down tree transformations.

In this section we show that the hierarchy $\left\{s l-D T^{n} \mid n \geq 1\right\}$ is proper. In fact, it will be the consequence of a stronger result, namely that the hierarchy of tree language classes $\left\{\operatorname{dom}\left(s l-D T^{n}\right) \mid n \geq 1\right\}$ is proper.

Thus first we prove that $\left\{\operatorname{dom}\left(s l-D T^{n}\right) \mid n \geq 1\right\}$ is proper. Our method is the following. For each $n \geq 2$, we define $n$ sl-dt tree transducers $T^{1, n}, \ldots, T^{n, n}$. Then we show that, for every $s \geq 1$ and arbitrary sl-dt tree transducers $M_{1}, \ldots, M_{s}$, if $\operatorname{dom}\left(\tau_{T^{1, n}} \circ \cdots \circ \tau_{T^{n, n}}\right)=\operatorname{dom}\left(\tau_{M_{1}} \circ \cdots \circ \tau_{M_{s}}\right)$, then $s \geq n$ necessarily holds.

Let $n \geq 2$ and define the ranked alphabets $\Sigma^{0, n}, \ldots, \Sigma^{n, n}$ as follows: 
(i) $\Sigma^{0, n}=\left\{\#, \sigma_{1}, \ldots, \sigma_{n}\right\}$, where \# has rank 0 and $\sigma_{j}$ has rank 2 , for each $1 \leq j \leq n$.

(ii) $\Sigma^{i, n}=\Sigma^{i-1, n}-\left\{\sigma_{i}\right\}=\left\{\#, \sigma_{i+1}, \ldots, \sigma_{n}\right\}$, for every $1 \leq i \leq n$.

Observe that $\Sigma^{n, n}=\{\#\}$.

Next, for every $1 \leq i \leq n$, define the sl-dt tree transducer

$$
T^{i, n}=\left(Q, \Sigma^{i-1, n}, \Sigma^{i, n}, q, R^{i, n}\right)
$$

where

- $Q=\left\{q, q^{\prime}\right\}$ and

- $R^{i, n}$ consists of the rules

$$
\begin{aligned}
& -q\left(\sigma_{i}\left(x_{1}, x_{2}\right)\right) \rightarrow q^{\prime}\left(x_{1}\right), \\
& -q^{\prime}\left(\sigma_{i}\left(x_{1}, x_{2}\right)\right) \rightarrow q\left(x_{2}\right), \\
& -q\left(\sigma_{j}\left(x_{1}, x_{2}\right)\right) \rightarrow \sigma_{j}\left(q\left(x_{1}\right), q\left(x_{2}\right)\right), \text { for every } j \text { with }(i+1) \leq j \leq n, \text { and } \\
& -q(\#) \rightarrow \# .
\end{aligned}
$$

Informally speaking, the tree transducer $T^{i, n}$ works as follows. Its input alphabet is $\left\{\#, \sigma_{i}, \ldots, \sigma_{n}\right\}$. Given an input tree $t$, the tree transducer $T^{i, n}$, starting with its initial state $q$, performs an identical tree transformation on each $\sigma_{j}$ with $i<j \leq n$.

When $T^{i, n}$ meets, in state $q$, a subtree $t^{\prime}$ of $t$ with root $\sigma_{i}$, then it can process $t^{\prime}$ if and only if the following two conditions hold:

- $t^{\prime}=\sigma_{i}\left(\sigma_{i}\left(t_{1}, t_{2}\right), t_{3}\right)$, for some input subtrees $t_{1}, t_{2}, t_{3}$.

- It can process $t_{2}$ starting in state $q$.

If this is the case, then the tree transducer $T^{i, n}$ deletes the two consecutive $\sigma_{i}$ 's together with the subtrees $t_{1}$ and $t_{3}$ from $t^{\prime}$ (and hence from $t$ ) and then processes $t_{2}$ with the state $q$.

We prove our theorem by inspecting how the sl-dt tree transducers $M_{1}, \ldots, M_{s}$ must work on some special trees. Therefore, for every $1 \leq i \leq n$, we define the trees $r_{1, i}, \ldots, r_{9, i}$ as can be seen in Figure 3.1.

For each $1 \leq i \leq n$, we introduce the notation $\tau_{i, n}=\tau_{T^{1, n}} \circ \ldots \circ \tau_{T^{i, n}}$. Moreover, we write $\tau_{n}$ for $\tau_{n, n}$ for brevity. It is an exercise to show that the following statement holds.

Statement 3.1.1 Let $n \geq 2$ be an integer. Then the following conditions hold:

- For every $i$ such that $1 \leq i \leq n$, 


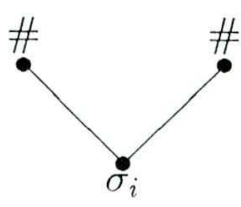

$r_{1, i}$

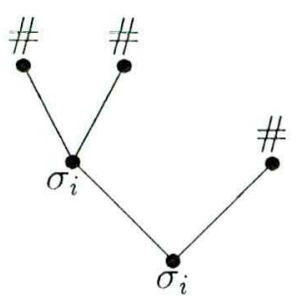

$r_{2, i}$

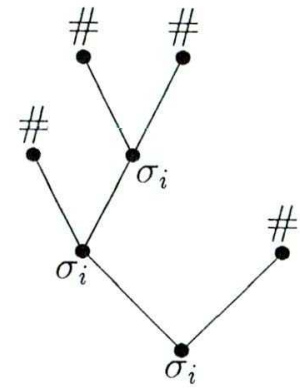

$r_{3, i}$

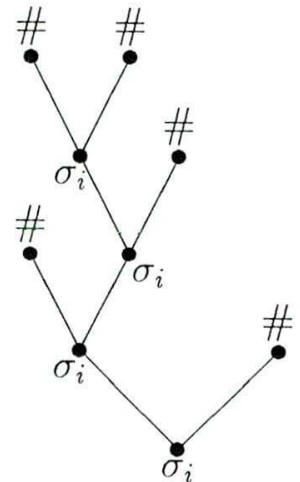

$r_{4, i}$

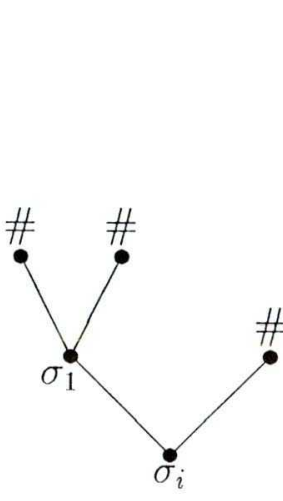

$r_{5, i}$

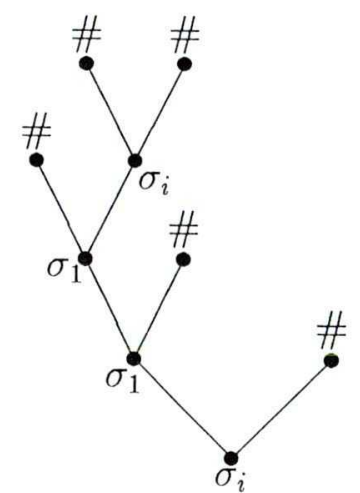

$r_{6, i}$

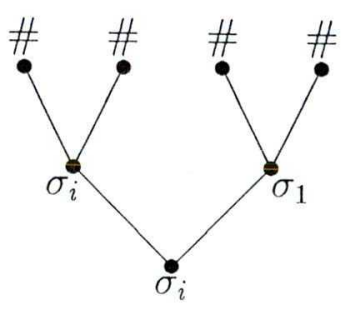

$r_{7, i}$

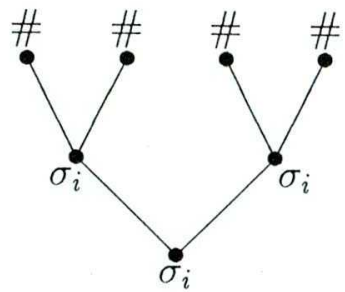

$r_{8, i}$

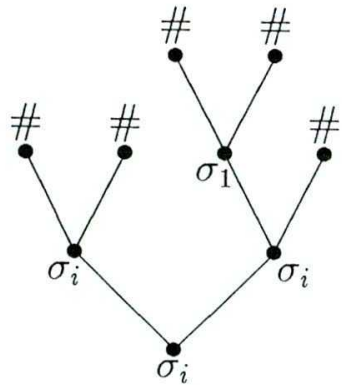

$r_{9, i}$

Figure 3.1: Example trees for hierarchy theorems 
- $r_{1, i}, r_{3, i} \notin \operatorname{dom}\left(\tau_{n}\right)$ and

- $r_{2, i}, r_{4, i} \in \operatorname{dom}\left(\tau_{n}\right)$.

- For every $i$ such that $2 \leq i \leq n$,

- $r_{5, i}, r_{7, i}, r_{9, i} \notin \operatorname{dom}\left(\tau_{n}\right)$ and

- $r_{6, i}, r_{8, i} \in \operatorname{dom}\left(\tau_{n}\right)$.

Now we are ready to prove the following lemma, which serves as the foundation of the results of this section.

Lemma 3.1.2 Let $n \geq 2$ and $s \geq 1$. Moreover, let $M_{1}, \ldots, M_{s}$ be an arbitrary sequence of sl-dt tree transducers and put $\mu_{s}=\tau_{M_{1}} \circ \cdots \circ \tau_{M_{s}}$. Then $\operatorname{dom}\left(\tau_{n}\right)=\operatorname{dom}\left(\mu_{s}\right)$ implies $s \geq n$.

Proof. We prove the lemma by induction on $n$.

The basis $n=2$. We prove by contradiction that $\operatorname{dom}\left(\tau_{2}\right)$ cannot be the domain of any sl-dt tree transformation. Therefore, suppose that there exists an sl-dt tree transducer

$$
T=\left(Q^{\prime}, \Sigma^{0,2}, \Delta, p, R\right)
$$

such that $\operatorname{dom}\left(\tau_{T}\right)=\operatorname{dom}\left(\tau_{2}\right)$.

We shall investigate the rules in $R$. Since \# $\in \operatorname{dom}\left(\tau_{2}\right)$, there must be a $(p, \#)$-rule in $R$, hence

$$
p(\#) \rightarrow t_{\#} \in R
$$

for some $t_{\#} \in T_{\Delta}$.

By Statement 3.1.1, $r_{2,2}$ is in $\operatorname{dom}\left(\tau_{2}\right)$. Therefore there must be a $\left(p, \sigma_{2}\right)$-rule in $R$. Moreover, since $T$ is linear, that $\left(p, \sigma_{2}\right)$-rule must be of one of the forms specified in (1)-(5). We shall show that each case leads to a contradiction.

(1) Assume that $p\left(\sigma_{2}\left(x_{1}, x_{2}\right)\right) \rightarrow t \in R$, where $t \in T_{\Delta}$ is a ground term. This implies $r_{1,2} \in \operatorname{dom}\left(\tau_{T}\right)$, which contradicts Statement 3.1.1.

(2) Assume that $p\left(\sigma_{2}\left(x_{1}, x_{2}\right)\right) \rightarrow t\left[p^{\prime}\left(x_{1}\right), p^{\prime \prime}\left(x_{2}\right)\right] \in R$, where $p^{\prime}, p^{\prime \prime} \in Q^{\prime}$ and $t \in \hat{T}_{\Delta, 2}$. Since $r_{1,2} \notin \operatorname{dom}\left(\tau_{2}\right)$, there cannot be $\left(p^{\prime}, \#\right)$ and $\left(p^{\prime \prime}, \#\right)$-rules in $R$ simultaneously. On the other hand, $r_{2,2}$ is in $\operatorname{dom}\left(\tau_{2}\right)$, thus a $\left(p^{\prime \prime}, \#\right)$-rule should be in $R$. Hence we can conclude that there is no $\left(p^{\prime}, \#\right)$-rule in $R$. Moreover, $r_{2,2} \in \operatorname{dom}\left(\tau_{2}\right)$ implies that there must be a $\left(p^{\prime}, \sigma_{2}\right)$-rule in $R$. Let this rule be specified as

$$
p^{\prime}\left(\sigma_{2}\left(x_{1}, x_{2}\right)\right) \rightarrow t^{\prime}
$$

where $t^{\prime} \in T_{\Delta}\left(Q\left(X_{2}\right)\right)$. Observe that the tree $t^{\prime}$ must contain the variable $x_{2}$, otherwise $r_{2,2} \in \operatorname{dom}\left(\tau_{T}\right)$ implies $r_{3,2} \in \operatorname{dom}\left(\tau_{T}\right)$, which contradicts 
Statement 3.1.1. Moreover, $T$ is superlinear and both $x_{1}$ and $x_{2}$ occur in $\operatorname{rhs}\left(p, \sigma_{2}\right)$. Therefore a variable can occur in $t^{\prime}=\operatorname{rhs}\left(p^{\prime}, \sigma_{2}\right)$ if and only if $p^{\prime}=p$. However, this is impossible, because there is a $(p, \#)$-rule in $R$, as we saw above.

(3) Suppose that $p\left(\sigma_{2}\left(x_{1}, x_{2}\right)\right) \rightarrow t\left[p^{\prime}\left(x_{2}\right), p^{\prime \prime}\left(x_{1}\right)\right] \in R$, where $p^{\prime}, p^{\prime \prime} \in Q^{\prime}$ and $t \in \hat{T}_{\Delta, 2}$. Observe that this case is quite similar to the previous one. The only difference is that the roles of $p^{\prime}$ and $p^{\prime \prime}$ are interchanged, hence such a rule in $R$ again implies a contradiction.

(4) Assume that $p\left(\sigma_{2}\left(x_{1}, x_{2}\right)\right) \rightarrow t\left[p^{\prime}\left(x_{2}\right)\right] \in R$, where $p^{\prime} \in Q^{\prime}$ and $t \in \hat{T}_{\Delta, 1}$. In this case, since the first subtree of $\sigma_{2}$ is deleted, the condition $r_{2,2} \in \operatorname{dom}\left(\tau_{T}\right)$ holds if and only if $r_{1,2} \in \operatorname{dom}\left(\tau_{T}\right)$, which contradicts Statement 3.1.1.

(5) Suppose that the rule $p\left(\sigma_{2}\left(x_{1}, x_{2}\right)\right) \rightarrow t\left[p^{\prime}\left(x_{1}\right)\right]$ is in $R$, where $p^{\prime} \in Q^{\prime}$ and $t \in \hat{T}_{\Delta, 1}$. Since the second subtree of $\sigma_{2}$ is deleted, we get $r_{2,2} \in \operatorname{dom}\left(\tau_{T}\right)$ if and only if $r_{7,2} \in \operatorname{dom}\left(\tau_{T}\right)$. This is contradiction, by Statement 3.1.1.

We have shown that no sl-dt tree transducer $T$ exists such that $\operatorname{dom}\left(\tau_{T}\right)=$ $\operatorname{dom}\left(\tau_{2}\right)$. Hence our lemma is proved for $n=2$.

Induction step. Suppose the lemma has been proved for $n-1$. Moreover, assume the sl-dt tree transducers $M_{1}, \ldots, M_{s}$ to be such that $\operatorname{dom}\left(\tau_{n}\right)=\operatorname{dom}\left(\mu_{s}\right)$.

By (4) of Corollary 2.1.12, an op-ni-sl-dt tree transducer $M_{1,1}$ and an nr-lnd-hom tree transducer $M_{1,2}$ exist such that $\tau_{M_{1}}=\tau_{M_{1,1}} \circ \tau_{M_{1,2}}$. Moreover, by Corollary 2.1.2 and by (3) of Corollary 2.1.12, the tree transducer $M_{2}^{\prime}=M_{1,2} \circ M_{2}$ is superlinear and $\tau_{M_{2}^{\prime}}=\tau_{M_{1,2}} \circ \tau_{M_{2}}$ holds.

Hence we have

$$
\begin{aligned}
\mu_{s} & =\tau_{M_{1,1}} \circ \tau_{M_{1,2}} \circ \tau_{M_{2}} \circ \tau_{M_{3}} \circ \cdots \circ \tau_{M_{s}} \\
& =\tau_{M_{1,1}} \circ \tau_{M_{2}^{\prime}} \circ \tau_{M_{3}} \circ \cdots \circ \tau_{M_{s}} .
\end{aligned}
$$

Roughly speaking, we "push forward" the undesirable properties, namely the variable permuting and the height increasing, by specializing the first tree transducer.

Suppose that

$$
M_{1,1}=\left(Q^{\prime}, \Sigma^{0, n}, \Omega, p, R\right) .
$$

Similarly to the case $n=2$, we investigate the rules of $R$. Since \# $\in \operatorname{dom}\left(\tau_{n}\right)$, there must be a $(p, \#)$-rule in $R$. Moreover, since $M_{1,1}$ is nonincreasing, this rule should be of the form

$$
p(\#) \rightarrow \#^{p},
$$

where $\#^{p} \in \Omega_{0}$.

Now let the integer $i$ be arbitrary but satisfying $2 \leq i \leq n$. Since $r_{2, i} \in$ $\operatorname{dom}\left(\tau_{n}\right)$, there must be a $\left(p, \sigma_{i}\right)$-rule in $R$. Moreover, $M_{1,1}$ is order preserving, 
nonincreasing and linear. Hence the $\left(p, \sigma_{i}\right)$-rule must be of one of the forms (1)-(4) detailed below.

(1) Assume that $p\left(\sigma_{i}\left(x_{1}, x_{2}\right)\right) \rightarrow \sigma_{i}^{p} \in R$, where $\sigma_{i}^{p} \in \Omega_{0}$. In this case, since the subtrees of $\sigma_{i}$ are deleted, $r_{2, i} \in \operatorname{dom}\left(\mu_{s}\right)$ if and only if $r_{1, i} \in \operatorname{dom}\left(\mu_{s}\right)$, which contradicts Statement 3.1.1. (Note that we have assumed $\operatorname{dom}\left(\tau_{n}\right)=$ $\operatorname{dom}\left(\mu_{s}\right)$.)

(2) Suppose that $p\left(\sigma_{i}\left(x_{1}, x_{2}\right)\right) \rightarrow t_{i}\left[p_{i}\left(x_{1}\right)\right] \in R$, where either $t_{i}=x_{1}$ or $t_{i}=$ $\sigma_{i}^{p}\left(x_{1}\right)$ holds for some $\sigma_{i}^{p} \in \Omega_{1}$ and $p_{i} \in Q^{\prime}$. The rule deletes the second subtree of $\sigma_{i}$, and therefore $r_{2, i} \in \operatorname{dom}\left(\mu_{s}\right)$ if and only if $r_{7, i} \in \operatorname{dom}\left(\mu_{s}\right)$, contradicting Statement 3.1.1.

(3) Assume that $p\left(\sigma_{i}\left(x_{1}, x_{2}\right)\right) \rightarrow t_{i}\left[p_{i}\left(x_{2}\right)\right] \in R$, where either $t_{i}=x_{1}$ or $t_{i}=$ $\sigma_{i}^{p}\left(x_{1}\right)$, for some $\sigma_{i}^{p} \in \Omega_{1}$ and $p_{i} \in Q^{\prime}$. Since the rule deletes the first subtree of $\sigma_{i}, r_{2, i} \in \operatorname{dom}\left(\mu_{s}\right)$ if and only if $r_{1, i} \in \operatorname{dom}\left(\mu_{s}\right)$, which contradicts Statement 3.1.1.

(4) We have obtained the fact that the only possible form for the $\left(p, \sigma_{i}\right)$-rule is

$$
p\left(\sigma_{i}\left(x_{1}, x_{2}\right)\right) \rightarrow \sigma_{i}^{p}\left(p_{i}\left(x_{1}\right), p_{i}^{\prime}\left(x_{2}\right)\right),
$$

where $\sigma_{i}^{p} \in \Omega_{2}$ and $p_{i}, p_{i}^{\prime} \in Q^{\prime}$.

Since $r_{8, i} \in \operatorname{dom}\left(\tau_{n}\right)$, both a $\left(p_{i}, \sigma_{i}\right)$-rule and a $\left(p_{i}^{\prime}, \sigma_{i}\right)$-rule must be in $R$. If $\operatorname{rhs}\left(p_{i}, \sigma_{i}\right)$ were a ground tree, then $r_{2, i} \in \operatorname{dom}\left(\mu_{s}\right)$ would imply $r_{3, i} \in \operatorname{dom}\left(\mu_{s}\right)$, which would contradict Statement 3.1.1.

Similarly, $\operatorname{rhs}\left(p_{i}^{\prime}, \sigma_{i}\right)$ cannot be a ground tree. Indeed, if it were, then $r_{8, i} \in$ $\operatorname{dom}\left(\mu_{s}\right)$ would imply $r_{9, i} \in \operatorname{dom}\left(\mu_{s}\right)$, contradicting Statement 3.1.1.

It follows that both $\operatorname{rhs}\left(p_{i}, \sigma_{i}\right)$ and $\operatorname{rhs}\left(p_{i}^{\prime}, \sigma_{i}\right)$ must contain a variable. However, since $M_{1,1}$ is superlinear, this is possible if and only if $p_{i}=p_{i}^{\prime}=p$. Hence we have shown that

$$
p\left(\sigma_{i}\left(x_{1}, x_{2}\right)\right) \rightarrow \sigma_{i}^{p}\left(p\left(x_{1}\right), p\left(x_{2}\right)\right) \in R .
$$

Recall that also $p(\#) \rightarrow \#^{p} \in R$.

We can suppose without loss of generality that the symbols $\#, \sigma_{2}, \ldots, \sigma_{n}$ are not in $\Omega$, which is the output ranked alphabet of $M_{1,1}$ and the input ranked alphabet of $M_{2}^{\prime}$. (Otherwise, we can easily relabel them both in $M_{1,1}$ and $M_{2}^{\prime}$ in such a way that the induced tree transformation $\tau_{M_{1,1}} \circ \tau_{M_{2}^{\prime}}$ remains the same.)

Then write \#, $\sigma_{2}, \ldots, \sigma_{n}$ for $\#^{p}, \sigma_{2}^{p}, \ldots, \sigma_{n}^{p}$, respectively, in $\Omega$ and both in the right-hand sides of rules of $M_{1,1}$ and in the left-hand sides of rules of $M_{2}^{\prime}$. We denote the resulting tree transducers by $\bar{M}_{1}$ and $\bar{M}_{2}$, respectively. It should be obvious that

$$
\tau_{M_{1,1}} \circ \tau_{M_{2}^{\prime}}=\tau_{\bar{M}_{1}} \circ \tau_{\bar{M}_{2}}
$$


and, by our results on the rules in $R,\left.\tau_{\bar{M}_{1}}\right|_{T_{\Sigma^{1, n}}}=\mathrm{id}\left(T_{\Sigma^{1, n}}\right)$. Consequently,

$$
\begin{aligned}
\operatorname{dom}\left(\left.\mu_{s}\right|_{T_{\Sigma^{1, n}}}\right) & =\operatorname{dom}\left(\left.\tau_{M_{1,1}} \circ \tau_{M_{2}^{\prime}} \circ \tau_{M_{3}} \circ \cdots \circ \tau_{M_{s}}\right|_{T_{\Sigma^{1, n}}}\right) \\
& =\operatorname{dom}\left(\left.\tau_{\bar{M}_{1}} \circ \tau_{\bar{M}_{2}} \circ \tau_{M_{3}} \circ \cdots \circ \tau_{M_{s}}\right|_{T_{\Sigma^{1, n}}}\right) \\
& =\operatorname{dom}\left(\left.\tau_{\bar{M}_{2}} \circ \tau_{M_{3}} \circ \cdots \circ \tau_{M_{s}}\right|_{T_{\Sigma^{1, n}}}\right) .
\end{aligned}
$$

Let the nr-l-hom tree transducer $T$ be such that $\tau_{T}=\mathrm{id}\left(T_{\Sigma^{1, n}}\right)$ holds. Obviously, there exists such a $T$, because $I \subseteq n r-l-H O M$ (see Subsection 1.4.1).

Consider the dt tree transducer $N_{2}$ defined by $N_{2}=T \circ \bar{M}_{2}$. Note that $T$ is total, superlinear, and nonreducing. Therefore, by Corollary 2.1 .2 and by (3) of Corollary 2.1.12, $N_{2}$ is superlinear and $\tau_{N_{2}}=\tau_{T} \circ \tau_{\bar{M}_{2}}$ holds. Hence we can compute on as follows:

$$
\begin{aligned}
\operatorname{dom}\left(\left.\tau_{\bar{M}_{2}} \circ \tau_{M_{3}} \circ \ldots \circ \tau_{M_{s}}\right|_{T_{\Sigma^{1, n}}}\right) & =\operatorname{dom}\left(\tau_{T} \circ \tau_{\bar{M}_{2}} \circ \tau_{M_{3}} \circ \cdots \circ \tau_{M_{s}}\right) \\
& =\operatorname{dom}\left(\tau_{N_{2}} \circ \tau_{M_{3}} \circ \cdots \circ \tau_{M_{s}}\right) .
\end{aligned}
$$

On the other hand, observe that $T^{1, n}$ and $T^{2, n}$ are defined so that $\left.\tau_{T^{1, n}}\right|_{T_{\Sigma^{1, n}}}=$ $\operatorname{id}\left(T_{\Sigma^{1, n}}\right)$ and $\operatorname{dom}\left(\tau_{T^{2, n}}\right) \subseteq T_{\Sigma^{1, n}}$. This implies

$$
\begin{aligned}
\operatorname{dom}\left(\left.\tau_{n}\right|_{T_{\Sigma^{1, n}}}\right) & =\operatorname{dom}\left(\left.\tau_{T^{1, n}} \circ \cdots \circ \tau_{T^{n, n}}\right|_{T_{\Sigma^{1, n}}}\right) \\
& =\operatorname{dom}\left(\tau_{T^{2, n}} \circ \cdots \circ \tau_{T^{n, n}}\right) .
\end{aligned}
$$

Hence, by $\operatorname{dom}\left(\left.\mu_{s}\right|_{T_{\Sigma^{1, n}}}\right)=\operatorname{dom}\left(\left.\tau_{n}\right|_{T_{\Sigma^{1, n}}}\right)$, we have

$$
\operatorname{dom}\left(\tau_{N_{2}} \circ \tau_{M_{3}} \circ \ldots \circ \tau_{M_{s}}\right)=\operatorname{dom}\left(\tau_{T^{2, n}} \circ \ldots \circ \tau_{T^{n, n}}\right) .
$$

Now we would like to apply our induction hypothesis to $(*)$. However, we cannot do that in the present form of $(*)$, because $T^{2, n}, \ldots, T^{n, n}$ appear in it instead of $T^{1, n-1}, \ldots, T^{n-1, n-1}$. Fortunately, we can get the required form by suitably relabeling in $T^{2, n}, \ldots, T^{n, n}$.

We can observe the following. For every $2 \leq i \leq n$, if we write $\sigma_{i-1}, \ldots, \sigma_{n-1}$ for $\sigma_{i}, \ldots, \sigma_{n}$, respectively, in the specification of $T^{i, n}$, then we get $T^{i-1, n-1}$. Hence (*) implies

$$
\operatorname{dom}\left(\tau_{N_{2}^{\prime}} \circ \tau_{M_{3}} \circ \cdots \circ \tau_{M_{s}}\right)=\operatorname{dom}\left(\tau_{T^{1, n-1}} \circ \cdots \circ \tau_{T^{n-1, n-1}}\right),
$$

where $N_{2}^{\prime}$ is obtained from $N_{2}$ by writing $\sigma_{1}, \ldots, \sigma_{n-1}$ for $\sigma_{2}, \ldots, \sigma_{n}$ in the input alphabet and in the left-hand sides of the rules of $N_{2}$.

We obtained that dom $\left(\tau_{n-1}\right)$ appears as the domain of the composition of $s-1$ sl-dt tree transformations. Then, the by induction hypothesis, $s-1 \geq n-1$, which implies $s \geq n$.

With this we finished the proof of the lemma.

On the basis of Lemma 3.1 .2 we can easily prove the following important results. 
Theorem 3.1.3 For any integer $n \geq 1$, the following inclusions hold:
(1) $\operatorname{dom}\left(\operatorname{sl}-D T^{n}\right) \subset \operatorname{dom}\left(s l-D T^{n+1}\right)$.
(2) $\operatorname{dom}\left(\operatorname{sl}-D T^{n}\right) \subset D R E C$.
(3) $s l-D T^{n} \subset s l-D T^{n+1}$.

\section{Proof.}

(1) Obviously, $\operatorname{dom}\left(s l-D T^{n}\right) \subseteq \operatorname{dom}\left(s l-D T^{n+1}\right)$. On the other hand, by Lemma 3.1.2, $\operatorname{dom}\left(\tau_{n+1}\right) \notin \operatorname{dom}\left(s l-D T^{n}\right)$.

(2) We recall from Section 1.4.5 that $\operatorname{dom}\left(l-D T^{n}\right)=D R E C$ holds, for every $n \geq 1$. Hence we have

$$
\operatorname{dom}\left(s l-D T^{n}\right) \subset \operatorname{dom}\left(s l-D T^{n+1}\right) \subseteq \operatorname{dom}\left(l-D T^{n+1}\right)=\operatorname{dom}(l-D T)=D R E C .
$$

(3) It should be clear, by the proof of (1), that $\tau_{n+1} \notin s l-D T^{n}$.

Finally we present the inclusion diagram of the classes $D T^{2}, D T, l-D T^{2}, l$ $D T$, and $s l-D T^{n}$ with $n \geq 1$ in Figure 3.2. The proper inclusions shown by the diagram follow from Theorems 2.1.4, 2.1.13, 3.1.3 and Corollary 2.1.14.

\subsection{The hierarchy $t-s l-D T^{n}$}

Total tree transducers are of special interest in the theory of tree transformations.

Namely, it is known from [Rou] that although the classes DT and $l-D T$ are not closed under the composition, the subclasses $t-D T$ and $t-l-D T$ of the corresponding total tree transformations are (see also Proposition 1.4.1). This explains our motivation to study the class $t-s l-D T$.

In the previous section we proved that $\left\{s l-D T^{n} \mid n \geq 1\right\}$ forms a proper hierarchy, which implies that $s l-D T$ is not closed under the composition. In this section we show that even the hierarchy $\left\{t-s l-D T^{n} \mid n \geq 1\right\}$ is proper.

We follow the method that we applied in the previous section. Namely, for each $n \geq 2$, we define $n$ t-sl-dt tree transducers $T^{1, n}, \ldots, T^{n, n}$. Then we show that, for every $s \geq 1$ and arbitrary t-sl-dt tree transducers $M_{1}, \ldots, M_{s}$, if $\tau_{T^{1, n}} \circ$ $\cdots \circ \tau_{T^{n, n}}=\tau_{M_{1}} \circ \cdots \circ \tau_{M_{s}}$, then $s \geq n$.

Therefore, let $n \geq 2$ be an arbitrary integer. Define the ranked alphabets $\Sigma^{0, n}, \ldots, \Sigma^{n, n}$ as follows:

(i) $\Sigma^{0, n}=\left\{\#, \$, \sigma_{1}, \ldots, \sigma_{n}\right\}$ where \# and $\$$ have rank 0 and $\sigma_{j}$ has rank 2 , for each $1 \leq j \leq n$.

(ii) $\sum^{i, n}=\Sigma^{i-1, n}-\left\{\sigma_{i}\right\}=\left\{\#, \$, \sigma_{i+1}, \ldots, \sigma_{n}\right\}$, for every $1 \leq i \leq n$.

It should be clear that $\Sigma^{n, n}=\{\#, \$\}$. 


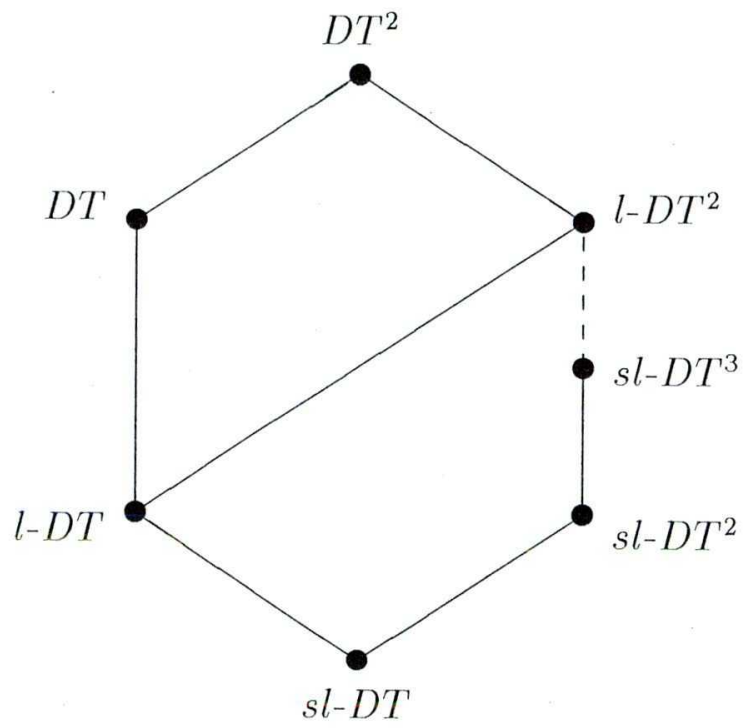

Figure 3.2: The hierarchy of $s l-D T^{n}$ 
Next, for every $1 \leq i \leq n$, define the $t$-sl-dt tree transducer

$$
T^{i, n}=\left(Q, \Sigma^{i-1, n}, \Sigma^{i, n}, q, R^{i, n}\right),
$$

where

- $Q=\left\{q, q^{\prime}\right\}$ and

- $R^{i, n}$ consists of the rules

- $q\left(\sigma_{i}\left(x_{1}, x_{2}\right)\right) \rightarrow q^{\prime}\left(x_{1}\right)$,

$-q^{\prime}\left(\sigma_{i}\left(x_{1}, x_{2}\right)\right) \rightarrow q\left(x_{2}\right)$,

- $q\left(\sigma_{j}\left(x_{1}, x_{2}\right)\right) \rightarrow \sigma_{j}\left(q\left(x_{1}\right), q\left(x_{2}\right)\right)$, for every $(i+1) \leq j \leq n$,

- $q^{\prime}\left(\sigma_{j}\left(x_{1}, x_{2}\right)\right) \rightarrow \$$, for every $(i+1) \leq j \leq n$, and

$-q(\#) \rightarrow \#, q(\$) \rightarrow \$, q^{\prime}(\#) \rightarrow \$, q^{\prime}(\$) \rightarrow \$$.

Roughly speaking, the t-sl-dt tree transducer $T^{i, n}$ works as follows. Its input alphabet is $\left\{\#, \$, \sigma_{i}, \ldots, \sigma_{n}\right\}$. Given an input tree $t$, the tree transducer $T^{i, n}$, starting with its initial state $q$, performs an identical tree transformation for each $\sigma_{j}$ with $i<j \leq n$. When $T^{i, n}$ meets, in state $q$, a subtree $t^{\prime}$ of $t$ with root $\sigma_{i}$, then two cases are possible.

Case 1: $t^{\prime}=\sigma_{i}\left(\sigma_{i}\left(t_{1}, t_{2}\right), t_{3}\right)$, for some input subtrees $t_{1}, t_{2}, t_{3}$. If this is the case, then $T^{i, n}$ deletes the two $\sigma_{i}$ 's together with the subtrees $t_{1}$ and $t_{3}$ from $t^{\prime}$ (and hence from $t$ ) and then processes $t_{2}$ with state $q$.

Case 2: $t^{\prime}$ has some other form (although its root is $\sigma_{i}$ ). Then $T^{i, n}$ transforms the tree $t^{\prime}$ to $\$$.

Again, we introduce the notation $\tau_{i, n}=\tau_{T^{1, n}} \circ \cdots \circ \tau_{T^{i, n}}$, for every $1 \leq i \leq n$. We write $\tau_{n}$ for $\tau_{n, n}$. Moreover, we again refer to the trees depicted in Figure 3.1.

The proof of the following statement is an easy exercise.

Statement 3.2.1 Let $n \geq 2$ be an integer. Then the following statements hold:

- For every $i$ such that $1 \leq i \leq n$,

$$
\begin{aligned}
& -\tau_{n}\left(r_{1, i}\right)=\tau_{n}\left(r_{3, i}\right)=\$ \text { and } \\
& -\tau_{n}\left(r_{2, i}\right)=\tau_{n}\left(r_{4, i}\right)=\# .
\end{aligned}
$$

- For every $i$ such that $2 \leq i \leq n$,

$$
\begin{aligned}
& -\tau_{n}\left(r_{5, i}\right)=\$ \text { and } \\
& -\tau_{n}\left(r_{6, i}\right)=\tau_{n}\left(r_{7, i}\right)=\tau_{n}\left(r_{8, i}\right)=\tau_{n}\left(r_{9, i}\right)=\# .
\end{aligned}
$$


We can observe the following. For every $1 \leq i \leq n, \tau_{i, n}(t)=\$$ implies that $t$ is not in the domain of the partial tree transformation corresponding to $\tau_{i, n}$, c.f. the previous section.

However, the converse is not true. For example, $\tau_{n}\left(r_{7, i}\right)=\#$ holds, despite $r_{7, i}$ being not in the domain of the partial tree transformation corresponding to $\tau_{n}$, see Statement 3.1.1.

Our key lemma now sounds as follows.

Lemma 3.2.2 Let $n \geq 2$ and $s \geq 1$. Moreover, let $M_{1}, \ldots, M_{s}$ be arbitrary t-sl-dt tree transducers and put $\mu_{s}=\tau_{M_{1}} \circ \cdots \circ \tau_{M_{s}}$. Then $\tau_{n}=\mu_{s}$, implies $s \geq n$.

Proof. We prove also this lemma by induction on $n$.

The basis $n=2$. We prove by contradiction that $\tau_{2}$ cannot be induced by any $\mathrm{t}$-sl-dt tree transducer. Therefore, assume that there is a t-sl-dt tree transducer

$$
T=\left(Q^{\prime}, \Sigma^{0,2}, \Sigma^{2,2}, p, R\right)
$$

such that $\tau_{T}=\tau_{2}$ holds.

Note that both the input and the output ranked alphabets of $T$ are determined by the condition $n=2$.

We investigate the rules of $R$. Since $\tau_{2}(\#)=\#$ and $\tau_{2}(\$)=\$$, the rules

$$
p(\#) \rightarrow \#
$$

and

$$
p(\$) \rightarrow \$
$$

must be in $R$.

Let $1 \leq i \leq 2$ and consider the $\left(p, \sigma_{i}\right)$-rule. Since the output ranked alphabet of $T$ contains symbols having rank 0 only, the $\left(p, \sigma_{i}\right)$-rule must be of one of the forms specified in (1)-(4):

(1) $p\left(\sigma_{i}\left(x_{1}, x_{2}\right)\right) \rightarrow \#$,

(2) $p\left(\dot{\sigma}_{i}\left(x_{1}, x_{2}\right)\right) \rightarrow \$$,

(3) $p\left(\sigma_{i}\left(x_{1}, x_{2}\right)\right) \rightarrow p_{i}\left(x_{1}\right)$

(4) $p\left(\sigma_{i}\left(x_{1}, x_{2}\right)\right) \rightarrow p_{i}\left(x_{2}\right)$

where $p_{i} \in Q^{\prime}$. Observe that, in cases (1), (2) and (4) the application of the $\left(p, \sigma_{i}\right)$ rule deletes the first subtree of $\sigma_{i}$. Therefore, in these cases, $\tau_{T}\left(r_{2, i}\right)=\tau_{T}\left(r_{1, i}\right)$ holds, which contradicts Statement 3.2.1. Hence we obtain that the only possible form is (3), that is to say

$$
p\left(\sigma_{i}\left(x_{1}, x_{2}\right)\right) \rightarrow p_{i}\left(x_{1}\right) \in R .
$$


Since $\tau_{2}\left(r_{1, i}\right)=\$$, we have

$$
p_{i}(\#) \rightarrow \$ \in R
$$

Thus the states $p$ and $p_{i}$ must be different.

Now consider the $\left(p_{i}, \sigma_{i}\right)$-rule. Suppose that $\operatorname{rhs}\left(p_{i}, \sigma_{i}\right)$ is a ground tree. Then it must be \#, since $\tau_{T}\left(r_{2, i}\right)=\#$. This, however, implies $\tau_{T}\left(r_{3, i}\right)=\#$, which contradicts Statement 3.2.1.

We obtain that $\operatorname{rhs}\left(p_{i}, \sigma_{i}\right)$ must contain a variable. Noticing that $T$ is superlinear and that $x_{1}$ already appears in $\operatorname{rhs}\left(p, \sigma_{i}\right)$, we get that the $\left(p_{i}, \sigma_{i}\right)$-rule must be of the form

$$
p_{i}\left(\sigma_{i}\left(x_{1}, x_{2}\right)\right) \rightarrow p_{i}^{\prime}\left(x_{2}\right),
$$

for some $p_{i}^{\prime} \in Q^{\prime}$. Since $\tau_{2}\left(r_{2, i}\right)=\#$,

$$
p_{i}^{\prime}(\#) \rightarrow \# \in R
$$

must hold, and therefore $p_{i} \neq p_{i}^{\prime}$.

Consider the $\left(p_{i}^{\prime}, \sigma_{i}\right)$-rule. If $\operatorname{rhs}\left(p_{i}^{\prime}, \sigma_{i}\right)$ were a ground tree, then it must be \#, because $\tau_{T}\left(r_{4, i}\right)=\#$. Then $\tau_{T}\left(r_{3, i}\right)=\#$ would follow, contradicting Statement 3.2 .1 .

Therefore, $\operatorname{rhs}\left(p_{i}^{\prime}, \sigma_{i}\right)$ must also contain a variable. However $T$ is superlinear, hence $\operatorname{rhs}\left(p_{i}^{\prime}, \sigma_{i}\right)$ can contain a variable if and only if $p_{i}^{\prime}=p$.

In summary, the rules

$$
\begin{gathered}
p(\#) \rightarrow \#, \\
p(\$) \rightarrow \$, \\
p_{i}(\#) \rightarrow \$, \\
p\left(\sigma_{i}\left(x_{1}, x_{2}\right)\right) \rightarrow p_{i}\left(x_{1}\right),
\end{gathered}
$$

and

$$
p_{i}\left(\sigma_{i}\left(x_{1}, x_{2}\right)\right) \rightarrow p\left(x_{2}\right)
$$

must be in $R$, for $i=1,2$. Moreover, $p \neq p_{i}$ holds.

Now consider the $\left(p_{2}, \sigma_{1}\right)$-rule. We observe that $\operatorname{rhs}\left(p_{2}, \sigma_{1}\right)$ must contain the variable $x_{1}$, otherwise $\tau_{T}\left(r_{5,2}\right)=\tau_{T}\left(r_{6,2}\right)$ contradicts Statement 3.2.1. On the other hand, $\operatorname{rhs}\left(p, \sigma_{1}\right)$ also contains $x_{1}$. Since $T$ is superlinear, we get $p=p_{2}$, which is a contradiction.

With this we proved that a suitable t-sl-dt tree transducer $T$ does not exist.

The induction step. Suppose the lemma has been proved for $n-1$. Moreover, assume the t-sl-dt tree transducers $M_{1}, \ldots, M_{s}$ to be such that $\mu_{s}=\tau_{n}$.

By (5) of Corollary 2.1.12, a t-op-ni-sl-dt tree transducer $M_{1,1}$ and a nr-l-ndhom tree transducer $M_{1,2}$ exist such that $\tau_{M_{1}}=\tau_{M_{1,1}} \circ \tau_{M_{1,2}}$. 
Define the tree transducer $M_{2}^{\prime}=M_{1,2} \circ M_{2}$. Observe that, by Lemma 2.1.11 and by (1) and (3) of Proposition 1.4.1, $M_{2}^{\prime}$ is t-sl-dt and $\tau_{M_{2}^{\prime}}=\tau_{M_{1,2}} \circ \tau_{M_{2}}$.

Hence we have the equations

$$
\begin{aligned}
\mu_{s} & =\tau_{M_{1}} \circ \tau_{M_{2}} \circ \tau_{M_{3}} \circ \cdots \circ \tau_{M_{s}} \\
& =\tau_{M_{1,1}} \circ \tau_{M_{2}^{\prime}} \circ \tau_{M_{3}} \circ \cdots \circ \tau_{M_{s}} .
\end{aligned}
$$

We specify $M_{1,1}$ as

$$
M_{1,1}=\left(Q^{\prime}, \Sigma^{0, n}, \Omega, p, R\right) .
$$

Let us examine the rules in $R$. Since $M_{1,1}$ is nonincreasing, the rules

$$
p(\#) \rightarrow \#^{p}
$$

and

$$
p(\$) \rightarrow \$^{p}
$$

are in $R$, for some $\#^{p}, \$^{p} \in \Omega_{0}$.

Now let $i$ be arbitrary such that $1 \leq i \leq n$. Consider the $\left(p, \sigma_{i}\right)$-rule. We observe that, since $M_{1,1}$ is linear, order preserving and nonincreasing, it must be of one of the following forms:

(1) $p\left(\sigma_{i}\left(x_{1}, x_{2}\right)\right) \rightarrow \sigma_{i}^{p}$, where $\sigma_{i}^{p} \in \Omega_{0}$.

(2) $p\left(\sigma_{i}\left(x_{1}, x_{2}\right)\right) \rightarrow t_{i}\left[p_{i}\left(x_{1}\right)\right]$, where $p_{i} \in Q^{\prime}$ and either $t_{i}=x_{1}$ or $t_{i}=\sigma_{i}^{p}\left(x_{1}\right)$, for some $\sigma_{i}^{p} \in \Omega_{1}$.

(3) $p\left(\sigma_{i}\left(x_{1}, x_{2}\right)\right) \rightarrow t_{i}\left[p_{i}\left(x_{2}\right)\right]$, where $p_{i} \in Q^{\prime}$ and either $t_{i}=x_{1}$ or $t_{i}=\sigma_{i}^{p}\left(x_{1}\right)$, for some $\sigma_{i}^{p} \in \Omega_{1}$.

(4) $p\left(\sigma_{i}\left(x_{1}, x_{2}\right)\right) \rightarrow \sigma_{i}^{p}\left(p_{i}\left(x_{1}\right), p_{i}^{\prime}\left(x_{2}\right)\right)$, where $p_{i}, p_{i}^{\prime} \in Q^{\prime}$ and $\sigma_{i}^{p} \in \Omega_{2}$.

We show that cases (1)-(3) lead to a contradiction.

Indeed, in cases (1) and (3), the first subtree of $\sigma_{i}$ is deleted. Hence, in both cases, $\mu_{s}\left(r_{1, i}\right)=\mu_{s}\left(r_{2, i}\right)$ holds, which contradicts Statement 3.2.1. (Note that we assumed $\mu_{s}=\tau_{n}$.)

Next, suppose that the case (2) holds. Consider the $\left(p_{i}, \sigma_{i}\right)$-rule. We observe that $\operatorname{rhs}\left(p_{i}, \sigma_{i}\right)$ must contain $x_{2}$. For if it does not, then $\mu_{s}\left(r_{2, i}\right)=\mu_{s}\left(r_{3, i}\right)$ holds, which contradicts Statement 3.2.1. This implies that $p \neq p_{i}$.

Since $M_{1,1}$ is a superlinear and nonincreasing tree transducer, the $\left(p_{i}, \sigma_{i}\right)$-rule must be of the form

$$
p_{i}\left(\sigma_{i}\left(x_{1}, x_{2}\right)\right) \rightarrow t_{i}^{\prime}\left[p_{i}^{\prime}\left(x_{2}\right)\right],
$$

where $p_{i}^{\prime} \in Q^{\prime}$ and either $t_{i}^{\prime}=x_{1}$ or $t_{i}^{\prime}=\sigma_{i}^{p_{i}}$, for some $\sigma_{i}^{p_{i}} \in \Omega_{1}$. The $\left(p_{i}^{\prime}, \sigma_{i}\right)$ rule must contain $x_{1}$ on its right-hand side, otherwise $\mu_{s}\left(r_{3, i}\right)=\mu_{s}\left(r_{4, i}\right)$ holds, contradicting Statement 3.2.1. However, since $M_{1,1}$ is superlinear, this is possible if and only if $p_{i}^{\prime}=p$. 
From now on, let $2 \leq i \leq n$ and consider the $\left(p_{i}, \sigma_{1}\right)$-rule. The tree $\operatorname{rhs}\left(p_{i}, \sigma_{1}\right)$ must contain $x_{1}$, otherwise $\mu_{s}\left(r_{5, i}\right)=\mu_{s}\left(r_{6, i}\right)$ contradicts Statement 3.2.1. On the other hand, the $\left(p, \sigma_{1}\right)$-rule also contains $x_{1}$ on its right-hand side. Since the tree transducer $M_{1,1}$ is superlinear, it follows that $p_{i}=p$, for every $2 \leq i \leq n$, which is a contradiction.

We got that the $\left(p, \sigma_{i}\right)$-rule cannot delete any of the subtrees of $\sigma_{i}$. Therefore its only possible form is (4), that is to say,

$$
p\left(\sigma_{i}\left(x_{1}, x_{2}\right)\right) \rightarrow \sigma_{i}^{p}\left(p_{i}\left(x_{1}\right), p_{i}^{\prime}\left(x_{2}\right)\right) \in R,
$$

where $p_{i}, p_{i}^{\prime} \in Q^{\prime}$ and $\sigma_{i}^{p} \in \Omega_{2}$. We show that $p_{i}=p_{i}^{\prime}=p$.

Really, $M_{1,1}$ is superlinear, hence if $p \neq p_{i}$, then $\operatorname{rhs}\left(p_{i}, \sigma_{i}\right)$ must be a ground tree, which implies the contradiction $\mu_{s}\left(r_{2, i}\right)=\mu_{s}\left(r_{3, i}\right)$. Consequently $p=p_{i}$, that is to say,

$$
p\left(\sigma_{i}\left(x_{1}, x_{2}\right)\right) \rightarrow \sigma_{i}^{p}\left(p\left(x_{1}\right), p_{i}^{\prime}\left(x_{2}\right)\right) \in R .
$$

However, in this case the assumption $p \neq p_{i}^{\prime}$ leads to the contradiction $\mu_{s}\left(r_{3, i}\right)=$ $\mu_{s}\left(r_{4, i}\right)$, hence $p_{i}^{\prime}=p$ holds, too.

We have obtained that the rules

$$
\begin{gathered}
p(\#) \rightarrow \#^{p}, \\
p(\$) \rightarrow \$^{p},
\end{gathered}
$$

and

$$
p\left(\sigma_{i}\left(x_{1}, x_{2}\right)\right) \rightarrow \sigma_{i}^{p}\left(p\left(x_{1}\right), p\left(x_{2}\right)\right)
$$

are in $R$.

Similarly as in the proof of Lemma 3.1.2, we can suppose without loss of generality that the symbols $\#, \$, \sigma_{2}, \ldots, \sigma_{n}$ do not occur in $\Omega$, which is the output alphabet of $M_{1,1}$ and the input alphabet of $M_{2}^{\prime}$.

Then write $\#, \$, \sigma_{2}, \ldots, \sigma_{n}$ for $\#^{p}, \$^{p}, \sigma_{2}^{p}, \ldots, \sigma_{n}^{p}$, respectively, in $\Omega$ and both in the right-hand sides of rules of $M_{1,1}$ and in the left-hand sides of rules of $M_{2}^{\prime}$. We denote the obtained t-sl-dt tree transducers by $\bar{M}_{1}$ and $\bar{M}_{2}$, respectively. Clearly, $\tau_{M_{1,1}} \circ \tau_{M_{2}^{\prime}}=\tau_{\bar{M}_{1}} \circ \tau_{\bar{M}_{2}}$ holds.

What we have shown about $R$ implies that the rules

$$
\begin{gathered}
p(\#) \rightarrow \#, \\
p(\$) \rightarrow \$,
\end{gathered}
$$

and

$$
p\left(\sigma_{i}\left(x_{1}, x_{2}\right)\right) \rightarrow \sigma_{i}\left(p\left(x_{1}\right), p\left(x_{2}\right)\right)
$$

will belong to $\bar{M}_{1}$. Hence $\bar{M}_{1}$ induces the identity tree transformation on $T_{\Sigma^{1, n}}$, that is to say, $\left.\tau_{\bar{M}_{1}}\right|_{T_{\Sigma^{1, n}}}=\mathrm{id}\left(T_{\Sigma^{1, n}}\right)$. 
From now on, the proof is very similar to that of Lemma 3.1.2, hence we omit the details.

Let $T$ be an nr-1-hom tree transducer such that $\tau_{T}=\mathrm{id}\left(T_{\Sigma^{1, n}}\right)$. Then we have

$$
\left.\mu_{s}\right|_{T_{\Sigma} 1, n}=\tau_{T} \circ \tau_{\bar{M}_{2}} \circ \tau_{M_{3}} \circ \cdots \circ \tau_{M_{s}}
$$

Now, let $N_{2}=T \circ \bar{M}_{2}$. Then $N_{2}$ is total, superlinear, and

$$
\tau_{T} \circ \tau_{\bar{M}_{2}} \circ \tau_{M_{3}} \circ \cdots \circ \tau_{M_{s}}=\tau_{N_{2}} \circ \tau_{M_{3}} \circ \cdots \circ \tau_{M_{s}} .
$$

On the other hand,

$$
\left.\tau_{n}\right|_{T_{\Sigma^{1, n}}}=\tau_{T^{2, n}} \circ \cdots \circ \tau_{T^{n, n}}
$$

Thus we get

$$
\tau_{\bar{M}_{2}} \circ \tau_{M_{3}} \circ \cdots \circ \tau_{M_{s}}=\tau_{T^{2, n}} \circ \cdots \circ \tau_{T^{n, n}} .
$$

Now we are able to apply the induction hypothesis. By a suitable relabeling, we obtain

$$
\tau_{N_{2}^{\prime}} \circ \tau_{M_{3}} \circ \cdots \circ \tau_{M_{s}}=\tau_{T^{1, n-1}} \circ \cdots \circ \tau_{T^{n-1, n-1}} .
$$

We have shown that $\tau_{n-1}$ appears as the composition of $s-1 \mathrm{t}$-sl-dt tree transformations. Then, by induction hypothesis, $s-1 \geq n-1$, yielding $s \geq n$.

With this we finished the proof of the lemma.

Using Lemma 3.2.2 it is easy to prove the main result of this section, which sounds as follows.

Theorem 3.2.3 For any integer $n \geq 1$,

$$
t-s l-D T^{n} \subset t-s l-D T^{n+1}
$$

holds.

Proof. It should be clear that $t-s l-D T^{n} \subseteq t-s l-D T^{n+1}$. Moreover, by Lemma 3.2.2, we have $\tau_{n+1} \notin t-s l-D T^{n}$.

We present the inclusion diagram of the tree transformation classes $t$ - $D T$, $t-l-D T$, and $t$-sl-DT $T^{n}$ with $n \geq 1$ in Figure 3.3. The proper inclusions shown by the diagram follow from Corollary 2.1.14 and Theorem 3.2.3. Note that this is in fact the total version of the diagram in Figure 3.2. 


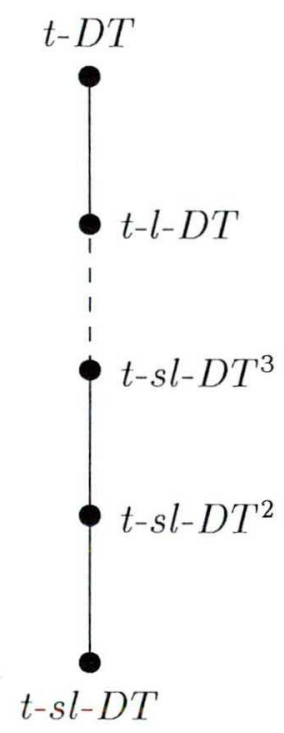

Figure 3.3: The hierarchy of $t-s l-D T^{n}$ 


\section{Chapter 4}

\section{Compositions with sl-dt tree transformations}

In this chapter we characterize the composition tree transformation classes of the form $X_{1} \circ \ldots \circ X_{n}$, where $n \geq 0$ and, for each $1 \leq i \leq n, X_{i}$ is the element of the set $M=\{H O M, s l-D T, l-D T, n d-D T, D T\}$. Namely, for arbitrary composition classes $\mathcal{C}_{1}$ and $\mathcal{C}_{2}$ of the above form, we want to know whether $\mathcal{C}_{1} \subseteq \mathcal{C}_{2}, \mathcal{C}_{1} \supseteq \mathcal{C}_{2}$, $\mathcal{C}_{1}=\mathcal{C}_{2}$, or $\mathcal{C}_{1} \bowtie \mathcal{C}_{2}$ holds.

Similar question was already investigated and a general method was proposed for solving these kind of problems in [FülVág4]. A description of that general method is also presented in [FülVág6]. In the works [FülVág2], [FülVág4], and [FülVág5] the general method was implemented for a set consisting of DT and six of its subclasses. Moreover, the method was also applied to a set consisting of deterministic bottom-up tree transformation classes in [Fü12], for a set of deterministic top-down tree transformation classes with regular look-ahead in [SluVág], and recently for a set consisting of deterministic bottom-up tree transformation classes and deterministic top-down tree transformation classes with and without regular look-ahead in [GyeVág].

In this chapter we slightly modify the general method and apply it to the set $M=\{H O M, s l-D T, l-D T, n d-D T, D T\}$. We chose this particular $M$ because we want to examine how the new class $s l-D T$ behaves when composing it with known deterministic top-down tree transformation classes.

We note that the results appearing in this chapter were published in the paper [DánFül2].

\subsection{The problem and the outline of the solution}

In this section we specify the problem that will be solved in the rest of the chapter. Moreover, we present the outline of the solution. 
In Section 2.1 we already stated several inclusions and decomposition equations concerning $s l-D T$. Such an inclusion and decomposition are $s l-D T \subset l-D T$ and $D T=n d-H O M \circ$ ol $-D T$, respectively.

On the other hand, several other inclusions and equations concerning tree transformation classes are known from the literature. For example we recall the inclusion $D T \subset D T^{2}$ from [Rou] and $H O M \circ l-D T=D T$ form [Eng3].

We observe that we can obtain some new inclusions and equations from the above ones, e.g. $n d-H O M \circ s l-D T \subset D T^{2}$ and $n d-H O M \circ s l-D T=H O M \circ l-D T$.

This observation motivates us to determine an abstract method, with which all inclusions and equations are derivable that are valid among tree transformation classes obtained from some base tree transformation classes by composition.

Since we are interested in the compositions of $s l-D T$ with the well known subclasses of DT, we shall choose $H O M, s l-D T, l-D T, n d-D T$, and $D T$ as base classes.

We now describe the problem in a more exact way. Let us fix the set

$$
M=\{H O M, \text { sl-DT, l-DT, nd-DT, DT }\} .
$$

We generalize the problem of inclusion and equality as follows. Whenever given two tree transformation classes

$$
X_{1} \circ X_{2} \circ \ldots \circ X_{m}
$$

and

$$
Y_{1} \circ Y_{2} \circ \ldots \circ Y_{n}
$$

such that $X_{i}, Y_{j} \in M$ for $1 \leq i \leq m$ and $1 \leq j \leq n$, we would like to know whether proper inclusion of some direction, equality or incomparability holds between them. Observe that we can answer the question if we can decide whether the inclusion

$$
X_{1} \circ X_{2} \circ \ldots X_{m} \subseteq Y_{1} \circ Y_{2} \circ \ldots Y_{n}
$$

holds or not. Really, if we can decide this inclusion, then we can also decide whether

$$
Y_{1} \circ Y_{2} \circ \ldots Y_{n} \subseteq X_{1} \circ X_{2} \circ \ldots X_{m}
$$

Then, for example,

$$
X_{1} \circ X_{2} \circ \ldots X_{m} \subset Y_{1} \circ Y_{2} \circ \ldots Y_{n}
$$

if and only if

$$
X_{1} \circ X_{2} \circ \ldots X_{m} \subseteq Y_{1} \circ Y_{2} \circ \ldots Y_{n}
$$

and

$$
Y_{1} \circ Y_{2} \circ \ldots Y_{n} \not X_{1} \circ X_{2} \circ \ldots X_{m}
$$


So we conclude that we have solved the problem if we present an algorithm which, for any two tree transformation classes as above, decides whether

$$
X_{1} \circ X_{2} \circ \ldots X_{m} \subseteq Y_{1} \circ Y_{2} \circ \ldots Y_{n}
$$

holds or not.

We now describe how this algorithm can be developed. Note that a general method was proposed for developing such an algorithm in [FülVág4]. The method presented here is a slight modification of that one.

Observe that the tree transformation classes of the form $X_{1} \circ X_{2} \circ \ldots \circ X_{m}$, where $m \geq 0$ and $X_{i} \in M$, for $1 \leq i \leq m$, constitute a monoid with the composition operation. The identity element of the monoid is $I$ resulting by the empty composition in case $m=0$. We denote this monoid by $[M]$. Hence our problem is to find an algorithm that decides the inclusion in $[M]$.

We also consider the free monoid $M^{*}$ generated by $M$ with the concatenation operation, which is denoted by - in this work. The identity relation over $M$ can uniquely be extended to a homomorphism ||$: M^{*} \rightarrow[M]$ (see [BurSan]). Then I| has the property that, for every element $X_{1} \cdot X_{2} \cdot \ldots \cdot X_{m}$ of $M^{*}$,

$$
\left|X_{1} \cdot X_{2} \cdot \ldots \cdot X_{m}\right|=X_{1} \circ X_{2} \circ \ldots \circ X_{m}
$$

holds. In particular, $|e|=I$. Let us denote the kernel of || by $\theta$. Then, certainly, for any two elements $X_{1} \circ X_{2} \circ \ldots \circ X_{m}$ and $Y_{1} \circ Y_{2} \circ \ldots \circ Y_{n}$ of $[M]$, we have

$$
X_{1} \circ X_{2} \circ \ldots \circ X_{m}=Y_{1} \circ Y_{2} \circ \ldots \circ Y_{n}
$$

in $[M]$ if and only if

$$
\left|X_{1} \cdot X_{2} \cdot \ldots \cdot X_{m}\right|=\left|Y_{1} \cdot Y_{2} \cdot \ldots \cdot Y_{n}\right|
$$

or equivalently

$$
X_{1} \cdot X_{2} \cdot \ldots \cdot X_{m} \theta Y_{1} \cdot Y_{2} \cdot \ldots \cdot Y_{n}
$$

in $M^{*}$.

Our algorithm is based on the following two corner stones.

1. First we present a confluent and terminating rewriting system $R \subseteq M^{*} \times M^{*}$ such that $\Leftrightarrow{ }_{R}^{*}=\theta$.

2. Second we present the inclusion diagram of the set

$$
|N F(R)|=\{|u| \mid u \in N F(R)\},
$$

where $N F(R)$ denotes the set of normal forms of $R$. 
Then, possessing the inclusion diagram for $|N F(R)|$, we can easily decide for any two normal forms $u, v \in N F(R)$ whether $|u| \subseteq|v|$ holds or not.

The algorithm works as follows. Let us be given two elements

$$
X_{1} \circ X_{2} \circ \ldots \circ X_{m}
$$

and

$$
Y_{1} \circ Y_{2} \circ \ldots \circ Y_{n}
$$

of $[M]$. Take the corresponding elements

$$
X_{1} \cdot X_{2} \cdot \ldots \cdot X_{m}
$$

and

$$
Y_{1} \cdot Y_{2} \cdot \ldots \cdot Y_{n}
$$

of $M^{*}$ and compute the normal forms $u, v \in N F(R)$ such that

$$
X_{1} \cdot X_{2} \cdot \ldots \cdot X_{m} \stackrel{\stackrel{*}{\Rightarrow}}{\Rightarrow} u
$$

and

$$
Y_{1} \cdot Y_{2} \cdot \ldots \cdot Y_{n} \underset{R}{\stackrel{*}{\Rightarrow}} v,
$$

respectively. Since $R$ is terminating and confluent, $u$ and $v$ exist and unique. Moreover,

$$
\left|X_{1} \cdot X_{2} \cdot \ldots \cdot X_{m}\right|=|u|
$$

and

$$
\left|Y_{1} \cdot Y_{2} \cdot \ldots \cdot Y_{n}\right|=|v|
$$

hold, because $\Rightarrow_{R}^{*} \subseteq \Leftrightarrow_{R}^{*}=\theta$ and $\theta$ is the kernel of || . Then, by the definition of I , the inclusion

$$
X_{1} \circ X_{2} \circ \ldots \circ X_{m} \subseteq Y_{1} \circ Y_{2} \circ \ldots \circ Y_{n}
$$

holds if and only if

$$
\left|X_{1} \cdot X_{2} \cdot \ldots \cdot X_{m}\right| \subseteq\left|Y_{1} \cdot Y_{2} \cdot \ldots \cdot Y_{n}\right|
$$

On the other hand, this latter inclusion is equivalent to $|u| \subseteq|v|$. Clearly, we can decide by direct inspection of the inclusion diagram whether $|u| \subseteq|v|$ holds or not. Hence we can also decide whether $(*)$ holds or not. 

(1) $l-D T^{2} \cdot H O M$
$\rightarrow \quad l-D T \cdot H O M$
(2) $\mathrm{HOM} \cdot \mathrm{HOM}$
$\rightarrow H O M$
(3) $D T \cdot H O M$
$\rightarrow D T^{2}$
(4) $s l-D T \cdot l-D T \cdot H O M$
$\rightarrow \quad l-D T \cdot H O M$
(5) $l-D T^{3}$
$\rightarrow \quad l-D T^{2}$
(6) $l-D T \cdot s l-D T$
$\rightarrow \quad l-D T^{2}$
(7) $l-D T \cdot D T$
$\rightarrow D T^{2}$
(8) $H O M \cdot l-D T$
$\rightarrow D T$
(9) $\mathrm{HOM} \cdot s l-D T$
$\rightarrow D T$
(10) $H O M \cdot D T$
$\rightarrow \quad D T$
(11) $D T \cdot l-D T$
$\rightarrow D T^{2}$
(12) $D T \cdot s l-D T$
$\rightarrow D T^{2}$
(13) $D T^{3}$
$\rightarrow D T^{2}$
(14) $s l-D T \cdot l-D T^{2}$
$\rightarrow \quad l-D T^{2}$
(15) $s l-D T \cdot D T^{2}$
$\rightarrow D T^{2}$
(16) $n d-D T \cdot H O M$
$\rightarrow D T^{2}$
(17) $n d-D T \cdot s l-D T$
$\rightarrow D T^{2}$
(18) $n d-D T \cdot l-D T$
$\rightarrow D T^{2}$
(19) $n d-D T \cdot n d-D T$
$\rightarrow n d-D T$
(20) $n d-D T \cdot D T$
$\rightarrow D T^{2}$
(21) $l-D T \cdot H O M \cdot n d-D T$
$\rightarrow \quad l-D T^{2} \cdot n d-D T$
(22) $D T \cdot n d-D T$
$\rightarrow \quad D T$

Figure 4.1: Rewriting rules of $R$ 


\subsection{The decidability of inclusions in the composition monoid}

Let us fix $M=\{H O M, s l-D T, l-D T, n d-D T, D T\}$ in the sequel. Recall that $\theta$ is the kernel of the homomorphism ||$: M^{*} \rightarrow[M]$, as defined in the previous section.

Let the term rewriting system $R$ over $M$ consist of the 22 rewriting rules enumerated in Figure 4.1.

In this section we present an algorithm, which decides the inclusion in $[M]$ in the way described in the previous section. We start by giving a rewriting system $R$ over $M^{*}$. Later on, we will show that $R$ is terminating, confluent and that $\Leftrightarrow{ }_{R}^{*}=\theta$.

First we show that, informally speaking, the strings in $M^{*}$, which are equivalent with respect to $\Leftrightarrow_{R}^{*}$, represent the same tree transformation class.

Lemma 4.2.1 The inclusion $\Leftrightarrow_{R}^{*} \subseteq \theta$ holds.

Proof. To prove the lemma it is sufficient to show that, for every $u \rightarrow v \in R$, we have $|u|=|v|$, or equivalently $u \theta v$. In words we say that the elements of $R$ are valid in $[M]$.

Indeed, if the elements of $R$ are valid in $[M]$, then $\Rightarrow_{R} \subseteq \theta$, which can be seen as follows. Let $w, z \in M^{*}$ such that $w \Rightarrow_{R} z$. Then, by the definition of $\Rightarrow_{R}$, there are strings $x$ and $y$ in $M^{*}$ and there is a rule $u \rightarrow v \in R$ so that $w=x \cdot u \cdot y$ and $z=x \cdot v \cdot y$. Then we can compute as follows:

$$
\begin{array}{rlr}
|w| & =|x \cdot u \cdot y| & \\
& =|x| \circ|u| \circ|y| & \text { (because }|| \text { is a homomorphism) } \\
& =|x| \circ|v| \circ|y| & \text { (because }|u|=|v| \text { ) } \\
& =|x \cdot v \cdot y| & \text { (because }|| \text { is a homomorphism) } \\
& =|z|, &
\end{array}
$$

proving that $w \theta z$.

Analogously, we can show that $\Rightarrow_{R}^{-1} \subseteq \theta$. Hence $\Leftrightarrow_{R} \subseteq \theta$ also holds. Finally, being $\theta$ the kernel of a homomorphism, we get $\Leftrightarrow_{R}^{*} \subseteq \theta^{*}=\theta$.

So it is enough to show that all elements of $R$ are valid in $[M]$. As a matter of fact, most of them were already proved in earlier works.

For instance, the validity of (8), i.e. that $H O M \circ l-D T=D T$ was proved in [Bak3] and [Eng1]. A lot of the others also follow implicitly from the results of [Bak3] and [Eng1]. However, we refer the reader to [FülVág1], because in that latter paper the proofs are explicit.

The validities of (2), (10), (19), and (22) are immediate consequences of Lemma 3 in [FülVág1]. Moreover, (5) and (13) are proved in Consequence 7, $(3),(7),(11),(16),(18)$, and (20) in Lemma 11 of the same paper. 
The equations (1) and (21) can be proved using Table 1 in [FülVág1] as follows. From Table 1, it turns out that $l-D T^{2} \circ H O M=l-n d-D T \circ H O M$ and also that $l-D T \circ H O M=l-n d-D T \circ H O M$, hence $l-D T^{2} \circ H O M=l-D T \circ H O M$. The validity of (21) can be shown similarly.

Note that the rest of the rules, namely (4), (6), (9),(12), (14), (15), and (17), all contain the class sl-DT, hence we should present the proofs of validity for them.

We can prove (4) as follows. Since $I \subseteq s l$-DT, it holds that $l-D T \circ H O M \subseteq$ sl-DT o $l-D T$ o HOM. As for the conversed inclusion, we have $s l-D T$ o $l-D T$ o $H O M \subseteq l-D T^{2} \circ H O M$, because $s l-D T \subseteq l-D T$. On the other hand (1) is valid, hence $l-D T^{2} \circ H O M=l-D T \circ H O M$, which proves the validity of (4).

For (6), we use $l-D T^{2}=l-D T$ ol-HOM (see Lemma 11 in [FülVág1]). Then $l-D T^{2}=l-D T \circ l-H O M \subseteq l-D T \circ s l-D T$, because $l-H O M=s l-H O M \subseteq s l-D T$, by Observation 2.1.1. Finally, $l-D T \circ s l-D T \subseteq l-D T^{2}$. These altogether prove the validity of $(6)$.

The validity of (9) follows from the equation $D T=n d-H O M$ o sl-DT (see Theorem 2.1.5), from the inclusion $n d-H O M \subseteq H O M$, and from the fact that homomorphism dt tree transformations are total (see (1) of Proposition 1.4.1).

Next, (12) can be shown quite similarly to the proof of (6), however here we must use the equation $D T^{2}=D T \circ l-H O M$, which comes again from Lemma 11 of [FülVág1].

The validities of (14) and (15) are obvious, because we saw that the equations (5) and (13) are valid.

Finally, (17) can be proved using $D T^{2}=n d-D T \circ l-H O M$, which was verified in Lemma 11 of [FülVág1].

Next we prove that $R$ is terminating. Recall that a weight reducing string rewriting system is necessarily terminating, see Section 1.2.

Lemma 4.2.2 The string rewriting system $R$ is terminating.

Proof. A weight function can easily be defined for $R$ so that $R$ is weight reducing. In fact, let $\rho: M \rightarrow\{1,2, \ldots\}$ be such that

$$
\begin{aligned}
& \rho(H O M)=3 \\
& \rho(s l-D T)=3 \\
& \rho(l-D T)=2 \\
& \rho(n d-D T)=2 \\
& \rho(D T)=1 \text {. }
\end{aligned}
$$

It is an easy exercise to check that $R$ is weight reducing. Hence it is terminating as well. 
We now give $N F(R)$, i.e. the set of $R$-normal forms. This happens in three steps. First we specify a set $N F \subseteq M^{*}$, second we present the inclusion diagram of the tree transformation classes represented by the elements of $N F$, and finally we show that $N F(R)=N F$.

As for the first step, let $N F$ be defined in the following way.

$$
\begin{aligned}
N F= & \left\{l-D T^{2}, l-D T \cdot H O M, l-D T^{2} \cdot n d-D T, D T^{2}\right\} \cup \\
& \left\{s l-D T^{n} \mid n \geq 0\right\} \cup \\
& \left\{s l-D T^{n} \cdot H O M \mid n \geq 0\right\} \cup \\
& \left\{s l-D T^{n} \cdot l-D T \mid n \geq 0\right\} \cup \\
& \left\{s l-D T^{n} \cdot n d-D T \mid n \geq 0\right\} \cup \\
& \left\{s l-D T^{n} \cdot l-D T \circ n d-D T \mid n \geq 0\right\} \cup \\
& \left\{s l-D T^{n} \cdot D T \mid n \geq 0\right\}
\end{aligned}
$$

Observe that $s l-D T^{0}=e$, hence $e \in N F$ holds.

We now present the inclusion diagram of the set of tree transformation classes, which are represented by the elements of $N F$.

Lemma 4.2.3 The diagram depicted in Figure 4.2 is the inclusion diagram of the set $|N F|=\{|u| \mid u \in N F\}$.

Proof. Actually, the involved diagram is a bit more than the inclusion diagram of $|N F|$, because it also contains the suprema of the six hierarchies appearing in $|N F|$. The reason of the insertion of these suprema is that the diagram becomes more complete and easier to handle in this a way.

The proof of the lemma is rather technical and tedious, hence we separated it into Section 4.3.

By the diagram in Figure 4.2, we have also that each element of $N F$ represents an unique tree transformation class.

Corollary 4.2.4 For any $u, w \in N F$, it holds that $|u|=|w|$ if and only if $u=w$.

Now we are ready to show that the set $N F$ defined above is exactly the set $N F(R)$ of $R$-normal forms.

Lemma 4.2.5 $N F(R)=N F$

Proof. It is easy, although tedious to show that $N F \subseteq N F(R)$. If we consider the elements of $N F$ one-by-one, then we can realize that there is no element such that a rule in Figure 4.1 is applicable to it.

The proof of $N F(R) \subseteq N F$ is strongly based on the tables depicted in Figures 4.3 and 4.4 , which are organized as follows. 


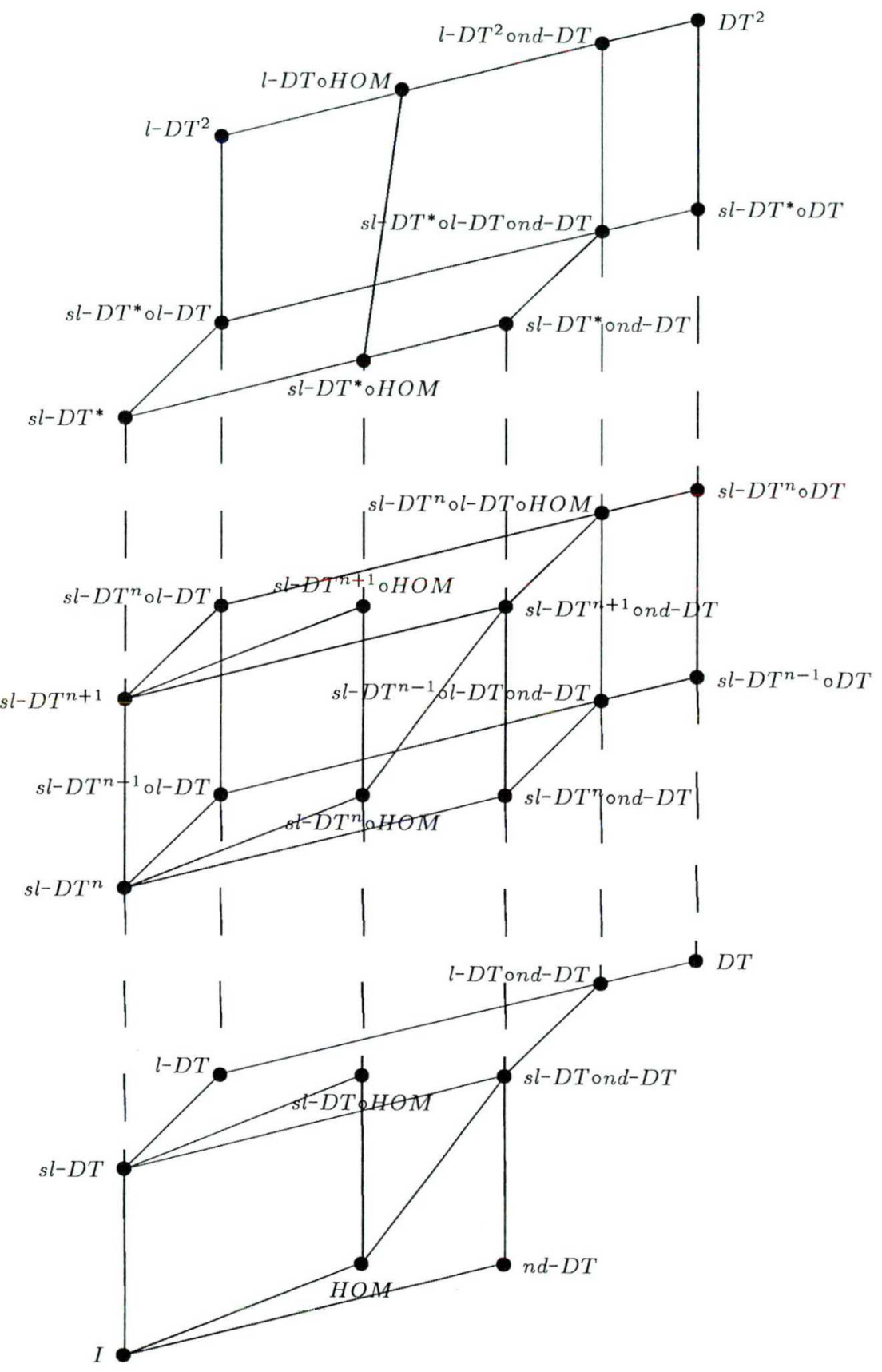

Figure 4.2: The inclusion diagram of normal forms 
In fact there is only one table, but it is divided into two parts because of space limitations. Therefore the tables in Figures 4.3 and 4.4 should be considered as one with 10 rows and 5 columns.

The 10 rows are labeled, on the one hand, by the 4 elements of the first union member forming $N F$ and, on the other hand, by the typical elements of the remaining 6 union members constituting $N F$. Moreover, the columns of the table are labeled by the elements of $M$.

We now describe how an entry determined by a row labeled by $u$ and a column labeled by $C$ is defined.

If $u \cdot C$ is also an element of $N F$, then the entry is exactly the word $u \cdot C$ and nothing else.

For example, if $u=s l-D T^{n} \cdot H O M$ and $C=n d-D T$, then the corresponding entry is $s l-D T^{n} \cdot H O M \cdot n d-D T$, because this latter is in $N F$ itself.

However, if $u \cdot C$ is not in $N F$, then the entry contains also an element $v$ of $N F$ and some number denoting rules in $R$ such that $u \cdot C \Rightarrow_{R}^{*} v$ by applying the rules appearing in the entry.

For instance, if $u=s l-D T^{n} \cdot l-D T$ and $C=s l-D T$, then the entry consists of $l-D T^{2}$ and the numbers (6) and (14), because $s l-D T^{n} \cdot l-D T \cdot s l-D T \Rightarrow_{R} s l-D T^{n}$. $l-D T^{2}$ by equation (6) and $s l-D T^{n} \cdot l-D T^{2} \Rightarrow_{R}^{*} l-D T^{2}$ by applying $n$ times the equation (14).

We now prove that, for every $x \in M^{*}$, the inclusion $x \in N F(R)$ implies $x \in N F$. The proof is performed by induction on length $(x)$.

Basis. If length $(x)=0$, then certainly $x=e$. Since $e \in N F$, we have nothing to prove.

Induction step. Now let $x \in M^{*}$ be such that length $(x)=n+1$ and suppose that the statement is true for every word in $M^{*}$ with length at most $n$. Then $x=y \cdot C$, for some $y \in M^{*}$ with length $n$ and $C \in M$. Assume that $x \in N F(R)$. Then, certainly, $y \in N F(R)$ (otherwise $x$ could not be in $N F(R)$ ) and thus, by the induction hypothesis, $y \in N F$, too.

Considering the definition of $N F, 10$ cases are possible, each of which corresponds to a row in the table, respectively. These are as follows.

Case 1: $y=l-D T^{2}$ and $n=2$. Then we can see from the table that $C$ can only be $n d-D T$, because in any other cases $x=y \cdot C=l-D T^{2} \cdot C$ can be reduced with some rules of $R$, hence could not be in $N F(R)$. (For example, in case $C=H O M$, $y \cdot C=l-D T^{2} \cdot H O M$ can be reduced to $l-D T \cdot H O M$ with rule (1), hence is not irreducible.) However, if $C=n d-D T$, then $x=y \cdot C=l-D T^{2} \cdot n d-D T$ is in $N F$, what we wanted to prove.

Cases 2,3,4: Here $y=l-D T \cdot H O M$ and $n=2, y=l-D T^{2} \cdot n d-D T$ and $n=3$, finally $y=D T^{2}$ and $n=2$, respectively. We can see easily from the table that, for every $y$ as above and each $C \in M, x=y \cdot C$ cannot be in $N F(R)$, hence we have nothing to prove. 
Case 5: $y=$ sl-DT $T^{n}$. We see from the table that, for every $C \in M, x=$ sl-DT $T^{n} \cdot C$ is in $N F$.

Since Cases 6-10 can be handled similarly, we left the rest part of the proof for an exercise.

Now we are ready to show that, roughly speaking, the strings in $M^{*}$ are equivalent with respect to $\Leftrightarrow_{R}^{*}$ if and only if represents the same tree transformation class.

Lemma 4.2.6 The equality $\theta=\Leftrightarrow_{R}^{*}$ holds.

Proof. Since we have already proved in Lemma 4.2.1 that $\Leftrightarrow_{R}^{*} \subseteq \theta$, it is sufficient to show the conversed inclusion $\theta \subseteq \Leftrightarrow_{R}^{*}$.

Indeed, let $x, y \in M^{*}$ be such that $x \theta y$. Since $R$ is terminating (see Lemma 4.2.2) there are words $u, v \in N F(R)$ such that $x \Rightarrow_{R}^{*} u$ and $y \Rightarrow_{R}^{*} v$.

However, again by Lemma 4.2.1, in this case $x \theta u$ and $y \theta v$ and thus $u \theta v$. On the other hand, by Lemma 4.2.5, $u$ and $v$ are also in $N F$. Hence, by Corollary 4.2.4, it holds that $u=v$.

We have obtained $x \Rightarrow_{R}^{*} u=v \Leftarrow_{R}^{*} y$ meaning that $x \Leftrightarrow_{R}^{*} y$. This finished the proof of the lemma.

Finally, we show that any word in $M^{*}$ can unambiguously be rewritten to an $R$-normal form.

Lemma 4.2.7 $R$ is confluent.

Proof. By Lemma 4.2.2, $R$ is terminating. Thus, by Proposition 1.1.25 in [Jan], it is sufficient to show that every $\Leftrightarrow_{R}^{*}$-class contains exactly one $R$-normal form. (For the proof of this fact, see also [BooOtt].)

Since $R$ is terminating, there is at least one normal form in every $\Leftrightarrow_{R^{-}}^{*}$ class. Let now $u, v \in N F$ such that $u \Leftrightarrow_{R}^{*} v$. Then, by $\Leftrightarrow_{R}^{*}=\theta$, we have $u \theta v$. However, by Corollary 4.2.4, this implies also $u=v$.

Now we are ready to prove the main result of this chapter, i.e. that the inclusion is decidable between any two composition classes consisting of the base tree transformation classes represented by the elcments of $M$.

Theorem 4.2.8 For any two tree transformation classes $X_{1} \circ X_{2} \circ \ldots \circ X_{m}$ and $Y_{1} \circ Y_{2} \circ \ldots \circ Y_{n}$ in $[M]$, it is decidable whether the inclusion $X_{1} \circ X_{2} \circ \ldots \circ X_{m} \subseteq$ $Y_{1} \circ Y_{2} \circ \ldots \circ Y_{n}$ holds.

Proof. Take the words $x=X_{1} \cdot X_{2} \cdot \ldots \cdot X_{m}$ and $y=Y_{1} \cdot Y_{2} \cdot \ldots \cdot Y_{n}$. Then, clearly, $|x|=X_{1} \circ X_{2} \circ \ldots \circ X_{m}$ and $|y|=Y_{1} \circ Y_{2} \circ \ldots \circ Y_{n}$. 


\begin{tabular}{|r||c|c|c|}
\hline & $H O M$ & $s l-D T$ & $l-D T$ \\
\hline \hline$l-D T^{2}$ & $l-D T \cdot H O M$ & $D T^{2}$ & $D T^{2}$ \\
& $(1)$ & $(6),(5)$ & $(5)$ \\
\hline$l-D T \cdot H O M$ & $l-D T \cdot H O M$ & $l-D T^{2}$ & $l-D T^{2}$ \\
& $(2)$ & $(9),(7)$ & $(8),(7)$ \\
\hline \multirow{2}{*}{$l-D T^{2} \cdot n d-D T$} & $D T^{2}$ & $D T^{2}$ & $D T^{2}$ \\
& $(16),(7),(13)$ & $(17),(7),(13)$ & $(18),(7),(13)$ \\
\hline \multirow{2}{*}{$D T^{2}$} & $D T^{2}$ & $D T^{2}$ & $D T^{2}$ \\
& $(3),(13)$ & $(12),(13)$ & $(11),(13)$ \\
\hline \multirow{2}{*}{$s l-D T^{n}$} & $s l-D T^{n} \cdot H O M$ & $s l-D T^{n+1}$ & $s l-D T^{n} \cdot l-D T$ \\
\hline \multirow{2}{*}{$s l-D T^{n} \cdot H O M$} & $s l-D T^{n} \cdot H O M$ & $s l-D T^{n} \cdot D T$ & $s l-D T^{n} \cdot D T$ \\
& $(2)$ & $(9)$ & $(8)$ \\
\hline \multirow{2}{*}{$s l-D T^{n} \cdot n d-D T$} & $l-D T \cdot H O M$ & $l-D T^{2}$ & $l-D T^{2}$ \\
& $(4)$ & $(6),(14)$ & $(14)$ \\
\hline \multirow{2}{*}{$s l-D T^{n} \cdot l-D T \cdot n d-D T$} & $(16),(15)$ & $(17),(15)$ & $(18),(15)$ \\
\hline \multirow{2}{*}{$s l-D T^{n} \cdot D T$} & $D T^{2}$ & $D T^{2}$ & $D T^{2}$ \\
& $(16),(7),(15)$ & $(17),(7),(15)$ & $(18),(7),(15)$ \\
\hline & $D T^{2}$ & $D T^{2}$ & $D T^{2}$ \\
& $(3),(15)$ & $(12),(15)$ & $(11),(15)$ \\
\hline
\end{tabular}

Figure 4.3: Table of concatenations with the elements in NF (part 1) 


\begin{tabular}{|c|c|c|}
\hline & $n d-D T$ & $D T$ \\
\hline$l-D T^{2}$ & $l-D T^{2} \cdot n d-D T$ & $\begin{array}{c}D T^{2} \\
(7),(13)\end{array}$ \\
\hline$l-D T \cdot H O M$ & $\begin{array}{c}l-D T^{2} \cdot n d-D T \\
(21)\end{array}$ & $\begin{array}{c}D T^{2} \\
(10),(7)\end{array}$ \\
\hline$l-D T^{2} \cdot n d-D T$ & $\begin{array}{c}l-D T^{2} \cdot n d-D T \\
(19)\end{array}$ & $\begin{array}{c}D T^{2} \\
(20),(7),(13)\end{array}$ \\
\hline$D T^{2}$ & $\begin{array}{c}l-D T^{2} \cdot n d-D T \\
(22)\end{array}$ & $\begin{array}{l}D T^{2} \\
(13)\end{array}$ \\
\hline$s l-D T^{n}$ & $s l-D T^{n} \cdot n d-D T$ & $s l-D T^{n} \cdot D T$ \\
\hline$s l-D T^{n} \cdot H O M$ & $s l-D T^{n} \cdot H O M \cdot n d-D T$ & $\begin{array}{c}s l-D T^{n} \cdot D T \\
(10)\end{array}$ \\
\hline$s l-D T^{n} \cdot l-D T$ & $s l-D T^{n} \cdot l-D T \cdot n d-D T$ & $\begin{array}{c}D T^{2} \\
(7),(15)\end{array}$ \\
\hline$s l-D T^{n} \cdot n d-D T$ & $\begin{array}{c}s l-D T^{n} \cdot n d-D T \\
(19)\end{array}$ & $\begin{array}{c}D T^{2} \\
(20),(15)\end{array}$ \\
\hline$s l-D T^{n} \circ l-D T \cdot n d-D T$ & $\begin{array}{c}s l-D T^{n} \cdot l-D T \cdot n d-D T \\
(19)\end{array}$ & $\begin{array}{c}D T^{2} \\
(20),(7),(15)\end{array}$ \\
\hline$s l-D T^{n} \cdot D T$ & $\begin{array}{c}s l-D T^{n} \cdot D T \\
(22)\end{array}$ & $\begin{array}{l}D T^{2} \\
(15)\end{array}$ \\
\hline
\end{tabular}

Figure 4.4: Table of concatenations with the elements in $N F$ (part 2) 
Let $u$ and $v$ be the $R$-normal forms of $x$ and $y$, respectively. Since $R$ is terminating and confluent, $u$ and $v$ are unambiguous and can be computed in linear time with respect to length $(x)$ and length $(y)$, just reducing $x$ and $y$ by $R$, respectively, as long as possible.

Then, by Lemma 4.2.6, also $x \theta u$ and $y \theta v$. Hence $|x| \subseteq|y|$ if and only if $|u| \subseteq|v|$. However, this latter inclusion is obviously decidable by inspecting the inclusion diagram of normal forms depicted in Figure 4.2.

We finish the section with an example. We would like to know the inclusion relation between the classes

$$
s l-D T^{3} \circ H O M \circ l-D T \text { o } n d-D T
$$

and

$$
\text { sl-DT } T^{2} \circ H O M \circ s l-D T \circ H O M \circ n d-D T .
$$

Then we compute as follows:

$$
\begin{array}{rll}
s l-D T^{3} \cdot H O M \cdot l-D T \cdot n d-D T & \Rightarrow_{R, 8} & s l-D T^{3} \cdot D T \cdot n d-D T \\
& \Rightarrow_{R, 22} & s l-D T^{3} \cdot D T,
\end{array}
$$

where we wrote $\Rightarrow_{R, i}$ to denote that we applied the $i$ th rule in that step of the computation. On the other hand,

$$
\begin{array}{rll}
s l-D T^{2} \cdot H O M \cdot s l-D T \cdot H O M \cdot n d-D T & \Rightarrow_{R, 9} & s l-D T^{2} \cdot D T \cdot H O M \cdot n d-D T \\
& \Rightarrow_{R, 3} & s l-D T^{2} \cdot D T^{2} \cdot n d-D T \\
& \Rightarrow_{R, 22} & s l-D T^{2} \cdot D T^{2} \\
& \Rightarrow_{R, 15}^{2} & D T^{2} .
\end{array}
$$

Finally, since both $s l-D T^{3} \cdot D T$ and $D T^{2}$ are in $N F$, we can see from the inclusion diagram in Figure 4.2 that $s l-D T^{3} \circ D T \subset D T^{2}$. Hence the relation $\subset$ holds between the two original classes we started with.

\subsection{The inclusion diagram of normal forms}

In this section we show that the inclusion diagram of $|N F|$ is exactly the diagram appearing in Figure 4.2.

We recall that $|N F|$ is the set of tree transformation classes represented by $N F$, which is the set of normal forms in $M^{*}$ with respect to the string rewriting system $R$, where $M$ and $R$ are defined at the beginning of Section 4.2.

To present the inclusion diagram we need some technical preparations.

Lemma 4.3.1 Let $n \geq 0$. Then

(1) $s l-D T^{n} \circ D T=$ op-ni-sl-DT $T^{n} \circ D T$ and

(2) $s l-D T^{n}$ ond-DT = op-ni-sl-DT $T^{n}$ ond-DT. 


\section{Proof.}

(1) Applying $n$ and $n-1$ times (4) and (6) of Corollary 2.1.12, respectively, and finally once (6) of Corollary 1.4 .2 to the left-hand side of the equation, we have exactly the right-hand side.

(2) Similarly, we get the right-hand side by applying (4) and (6) of Corollary 2.1.12 and (7) of Corollary 1.4.2

We shall also need the following stronger version of (2) of Theorem 3.1.3.

\section{Theorem 4.3.2 $\operatorname{dom}\left(s l-D T^{*}\right) \subset D R E C$}

Proof. Let $\Sigma=\left\{\sigma^{(1)}, \#^{(0)}\right\}$. Define the tree language

$$
L=\left\{\sigma^{i}(\#) \mid i \geq 0 \text { is an even integer }\right\}
$$

over $\Sigma$. Informally speaking, $L$ is the set of even-length chains over $\Sigma$. Note that obviously $L \in D R E C$. We prove $L \notin \operatorname{dom}\left(s l-D T^{*}\right)$.

To see this, suppose the contrary, i.e. that $L \in \operatorname{dom}\left(s l-D T^{n}\right)$, for some $n \geq 1$. Then there are sl-dt tree transducers $T_{1}, \ldots, T_{n}$, such that $L=\operatorname{dom}\left(\tau_{T_{1}} \circ \ldots \circ \tau_{T_{n}}\right)$. We can assume without loss of generality that $n$ is minimal.

Let $T_{1}=(Q, \Sigma, \Delta, q, R)$. We investigate the rules in $R$. Since $\# \in L$, there must be a rule of the form

$$
q(\#) \rightarrow t_{\#}
$$

in $R$, for some $t_{\#} \in T_{\Delta}$.

The tree $\sigma(\sigma(\#))$ is also in $L$, hence there must be a $(q, \sigma)$-rule in $R$. It is easy to see that $\operatorname{rhs}(q, \sigma)$ cannot be a ground tree.

On the other hand, $T_{1}$ is linear, hence the $(q, \sigma)$-rule is of the form $q\left(\sigma\left(x_{1}\right)\right) \rightarrow$ $t\left[q^{\prime}\left(x_{1}\right)\right]$ in $R_{1}$, for some $q^{\prime} \in Q$ and $t \in \hat{T}_{\Sigma, 1}$.

Similarly to the previous argumentation, it is easy to show that there must be a rule of the form $q^{\prime}\left(\sigma\left(x_{1}\right)\right) \rightarrow t^{\prime}\left[q^{\prime \prime}\left(x_{1}\right)\right]$ in $R$, where $q^{\prime \prime} \in Q$ and $t^{\prime} \in \hat{T}_{\Sigma, 1}$.

However, since $T_{1}$ is superlinear, this is possible if and only if $q=q^{\prime}=q^{\prime \prime}$, meaning that

$$
q\left(\sigma\left(x_{1}\right)\right) \rightarrow t\left[q\left(x_{1}\right)\right] \in R .
$$

Since $\Sigma=\{\sigma, \#\}$, there cannot be other useful rules in $R$. We obtained that $T_{1}$ is total, which implies $\operatorname{dom}\left(T_{1}\right)=T_{\Sigma}$. Hence, $n>1$ must hold.

Consider the above $(q, \sigma)$-rule of $R$. It is easy to see that $t=x_{1}$ would imply $\tau_{T_{1}}\left(\sigma^{i}(\#)\right)=t_{\#}$ for every $i \geq 0$. Hence $t \neq x_{1}$, meaning that $T_{1}$ is nonreducing.

We now have that $T_{1}$ is t-nr-sl-dt, that is $L \in \operatorname{dom}\left(t-n r-s l-D T \circ s l-D T^{n-1}\right)$, where $n>1$. Hence, by (3) of Corollary 2.1.12, $L \in \operatorname{dom}\left(s l-D T^{n-1}\right)$ holds, which contradicts that $n$ is minimal.

Moreover, we prove two technical, but very useful lemmas before considering the inclusion diagram of $|N F|$. 
We know from Section 3.1 that any sequence of sl-dt tree transducers has "low" transformation power. Roughly speaking, the first lemma shows that the transformation power does not increase significantly even if such a sequence is followed by a dt tree transducer.

Lemma 4.3.3 Let $L \subset T_{\Sigma}$ for some ranked alphabet $\Sigma$ and let $\sigma^{(2)}$, \#(0), and $\$^{(0)}$ be new ranked symbols. Put $\Sigma^{\prime}=\Sigma \cup\left\{\sigma^{(2)}, \#^{(0)}, \$^{(0)}\right\}$ and $\Delta=\left\{\#^{(0)}, \$^{(0)}\right\}$. Define the tree transformation $\tau \subseteq T_{\Sigma^{\prime}} \times T_{\Delta}$ as

$$
\tau=\left\{(\sigma(t, s), s) \mid t \in L, s \in T_{\Delta}\right\}
$$

If $\tau \in$ sl-DT $T^{n}$ oDT for some $n \geq 1$, then $L \in \operatorname{dom}\left(\right.$ sl-DT $\left.T^{m}\right)$ holds for some $m$ such that $1 \leq m \leq n$.

Proof. For brevity, we put $K=T_{\Delta}=\{\#, \$\}$. Observe that $\operatorname{dom}(\tau)=$ $\sigma(L, K)$.

Since $\tau \in$ sl-DT $T^{n} \circ D T$, the inclusion $\tau \in$ op-ni-sl-DT $T^{n} \circ D T$ holds by (1) of Lemma 4.3.1. That is, op-ni-sl-dt tree transducers $T_{1}, \ldots, T_{n}$ and a dt tree transducer $T_{n+1}$ exist such that $\tau=\tau_{T_{1}} \circ \ldots \circ \tau_{T_{n}} \circ \tau_{T_{n+1}}$.

Put $T_{i}=\left(Q_{i}, \Delta^{(i-1)}, \Delta^{(i)}, q, R_{i}\right)$, where $1 \leq i \leq n+1$. Observe that $\Delta^{(0)}=\Sigma^{\prime}$ and $\Delta^{(n+1)}=\Delta$. Moreover, we can assume without loss generality that the initial state of all $T_{i}$ 's is $q$.

Consider the dt tree transducer $T_{n+1}$. Its output alphabet is $\Delta$, which consists of symbols having rank 0 . Therefore, each rule of $T_{n+1}$ either must be a reducing one or it has $\$$ or $\#$ on its right-hand side. That is, $T_{n+1}$ should be an op-ni-l-dt tree transducer. Hence $\tau \in o p-n i-s l-D T^{n}$ o op-ni-l-DT.

For every $1 \leq i \leq n+1$, we define the type of the sequence $T_{1}, \ldots, T_{i}$ by induction on $i$. This type can be (1), (2) or undefined.

(i) The type of $T_{1}$ is

- (1) if there is a rule of the form

$$
q\left(\sigma\left(x_{1}, x_{2}\right)\right) \rightarrow \sigma_{1}\left(p_{1}\left(x_{1}\right), q_{1}\left(x_{2}\right)\right)
$$

in $R_{1}$, where $p_{1}, q_{1} \in Q_{1}$ and $\sigma_{1} \in \Delta_{2}^{(1)}$,

- (2) if there is a rule of the form

$$
q\left(\sigma\left(x_{1}, x_{2}\right)\right) \rightarrow \sigma_{1}\left(q_{1}\left(x_{2}\right)\right)
$$

in $R_{1}$, where $q_{1} \in Q_{1}$ and $\sigma_{1} \in \Delta_{1}^{(1)} \cup\left\{x_{1}\right\}$, and

- undefined otherwise.

Note that $q$ is the initial state of $T_{1}$. 
(ii) Let $i \geq 2$. Assume that the type of the sequence $T_{1}, \ldots, T_{i-1}$ has already been defined. The type of $T_{1}, \ldots, T_{i}$ is

- (1) if the type of $T_{1}, \ldots, T_{i-1}$ is (1) and there is a rule of the form

$$
q\left(\sigma_{i-1}\left(x_{1}, x_{2}\right)\right) \rightarrow \sigma_{i}\left(p_{i}\left(x_{1}\right), q_{i}\left(x_{2}\right)\right)
$$

in $R_{i}$, where $p_{i}, q_{i} \in Q_{i}$ and $\sigma_{i} \in \Delta_{2}^{(i)}$,

- (2) if the type of $T_{1}, \ldots, T_{i-1}$ is (1) and there is a rule of the form

$$
q\left(\sigma_{i-1}\left(x_{1}, x_{2}\right)\right) \rightarrow \sigma_{i}\left(q_{i}\left(x_{2}\right)\right)
$$

in $R_{i}$, where $q_{i} \in Q_{i}$ and $\sigma_{i} \in \Delta_{1}^{(i)} \cup\left\{x_{1}\right\}$, and

- undefined otherwise.

Note that here $q$ is the initial state of $T_{i}$.

We finish the proof as follows. First we make two observations. Observation 1 is on the domains of tree transformations induced by $T_{1}, \ldots, T_{i}$ sequences defined above. Then, in Observation 2, we characterize the tree transformations induced by sequences of types (1) and (2).

Following this, in Step 1 we show that if there is an integer $i$ with $1<i \leq n+1$ such that $T_{1}, \ldots, T_{i}$ is of type (2), then $L=\operatorname{dom}\left(\tau_{M_{1}} \circ \ldots \circ \tau_{M_{i-1}}\right)$ holds for some sl-dt tree transducers $M_{1}, \ldots, M_{i-1}$.

Finally, in Step 2, we prove that actually there is a sequence $T_{1}, \ldots, T_{i}$ of type (2), for some $1<i \leq n+1$.

Observation 1. Consider the tree transducer $T_{1}$. Since the root of each input tree in the tree translation $\tau$ is $\sigma$, there must be a $(q, \sigma)$-rule in $R_{1}$.

On the other hand, since $\sigma$ appears only as root in the input trees of $\tau$, we can suppose without loss of generality that this is the only rule containing the state $q$. (Otherwise, we take a new initial state for $T_{1}$.) Then we can also suppose that $\operatorname{dom}\left(\tau_{T_{1}}\right)=\sigma\left(L_{1}, K_{1}\right)$ holds for some $L_{1}, K_{1} \subseteq T_{\Sigma^{\prime}}$.

Clearly, $\operatorname{dom}\left(\tau_{T_{1}} \circ \ldots \circ \tau_{T_{i+1}}\right) \subseteq \operatorname{dom}\left(\tau_{T_{1}} \circ \ldots \circ \tau_{T_{i}}\right)$, for every $1 \leq i \leq n$. Hence we get $\operatorname{dom}\left(\tau_{T_{1}} \circ \ldots \circ \tau_{T_{i}}\right)=\sigma\left(L_{i}, K_{i}\right)$, for each $i$ such that $1 \leq i \leq n+1$. Moreover, $L_{i+1} \subseteq L_{i}, K_{i+1} \subseteq K_{i}$, for every $1 \leq i \leq n$. Specially, $L_{n+1}=L$ and $K_{n+1}=K$.

Observation 2. Let $1 \leq i \leq n+1$. If the sequence $T_{1}, \ldots, T_{i}$ is of type (1), then $\tau_{T_{1}} \circ \ldots \circ \tau_{T_{i}}$ consists of all pairs of the form $\left(\sigma(t, s), \sigma_{i}\left(t^{\prime}, s^{\prime}\right)\right)$, where exist trees $t_{0}, s_{0} \in T_{\Delta^{(0)}}, \ldots, t_{i}, s_{i} \in T_{\Delta(i)}$ such that

- $t_{0}=t, s_{0}=s, t_{i}=t^{\prime}, s_{i}=s^{\prime}$, and,

- for every $1 \leq j \leq i, p_{j}\left(t_{j-1}\right) \Rightarrow_{T_{j}}^{*} t_{j}$ and $q_{j}\left(s_{j-1}\right) \Rightarrow_{T_{j}}^{*} s_{j}$ 
hold. Note that $\sigma_{i}$ and the states $p_{1}, q_{1}, \ldots, p_{i}, q_{i}$ are defined in the definition of the sequence of type (1).

Now suppose that the sequence $T_{1}, \ldots, T_{i}$ is of type (2). Then $\tau_{T_{1}} \circ \ldots \circ \tau_{T_{i}}$ is the set of all pairs $\left(\sigma(t, s), \sigma_{i}\left(s^{\prime}\right)\right)$, where exist trees $t_{0}, s_{0} \in T_{\Delta^{(0)}}, \ldots, t_{i-1}, s_{i-1} \in$ $T_{\Delta^{(i-1)}}, s_{i} \in T_{\Delta^{(i)}}$ such that

- $t_{0}=t, s_{0}=s, s_{i}=s^{\prime}$,

- for every $1 \leq j \leq i-1, p_{j}\left(t_{j-1}\right) \Rightarrow_{T_{j}}^{*} t_{j}$ and $q_{j}\left(s_{j-1}\right) \Rightarrow_{T_{j}}^{*} s_{j}$, and

- $q_{i}\left(s_{i-1}\right) \Rightarrow_{T_{i}}^{*} s_{i}$

hold.

Step1. By the above observations, it is easy to see that if $T_{1}, \ldots, T_{i}$ is of type (2), for some $1 \leq i \leq n+1$, then $\operatorname{dom}\left(\tau_{T_{1}} \circ \ldots \circ \tau_{T_{i}}\right)=\sigma\left(L_{i-1}, K_{i}\right)$. Moreover, for every $j$, such that $i \leq j \leq n+1$, $\operatorname{dom}\left(\tau_{T_{1}} \circ \ldots \circ \tau_{T_{j}}\right)=\sigma\left(L_{i-1}, K_{j}\right)$ should hold.

On the other hand, $\operatorname{dom}\left(\tau_{T_{1}} \circ \ldots \circ \tau_{T_{n+1}}\right)=\operatorname{dom}(\tau)=\sigma(L, K)$, hence we have obtained that $L_{i-1}=L$. This provides that $T_{1}$ cannot be of type (2). If it were, then $L=T_{\Sigma^{\prime}}$ would follow, which is a contradiction.

Now, for each $1 \leq j \leq i-1$, let $M_{j}=\left(Q_{j}, \Delta^{(j-1)}, \Delta^{(j)}, p_{j}, R_{j}\right)$ be constructed from $T_{j}$ such that we let $p_{j}$ be the initial state instead of $q$. (Recall that the sequence $T_{1}, \ldots, T_{i-1}$ is of type (1), hence there must be a rule of the form $q\left(\sigma_{j-1}\left(x_{1}, x_{2}\right)\right) \rightarrow \sigma_{j}\left(p_{j}\left(x_{1}\right), q_{j}\left(x_{2}\right)\right)$ in $\left.R_{j}.\right)$ By the result of the previous paragraph, in this case $\operatorname{dom}\left(\tau_{M_{1}} \circ \ldots \circ \tau_{M_{i-1}}\right)=L_{i-1}=L$ holds. Note that $T_{j}$ is a superlinear tree transducer, hence so $M_{j}$ is.

Step 2. Consider the tree transducer $T_{1}$. By the definition of $\tau$, it is obvious that $T_{1}$, as a sequence, cannot be of type undefined. We have seen that it cannot be of type (2) either, hence it is of type (1).

Let $1 \leq i \leq n$. Suppose that $T_{1}, \ldots, T_{i}$ is of type (1). By Observation 2, it is easy to see that the tree transducer $T_{i+1}$ should have a $\left(q, \sigma_{i}\right)$-rule. Moreover, by the definition of $\tau, \operatorname{rhs}\left(q, \sigma_{i}\right)$ should contain $x_{2}$. Otherwise, roughly speaking, $T_{i+1}$ would "loose information" about the second direct subtree of the input tree.

Recall that $T_{i+1}$ is order preserving, nonincreasing and linear, and hence $T_{1}, \ldots, T_{i+1}$ must be of type (1) or (2).

Finally, we show that the whole sequence $T_{1}, \ldots, T_{n+1}$ cannot be of type (1). This follows from the fact that the output alphabet $\Delta^{n+1}$ of $T_{n+1}$ consists of symbols having rank 0 .

Hence there is a sequence $T_{1}, \ldots, T_{i}$ of type (2), for some $1 \leq i \leq n+1$.

Now we apply the previous lemma. To present the inclusion diagram of $|N F|$, we shall need the following results. 
Corollary 4.3.4 Let $n \geq 0$ integer. Then
(1) $s l-D T^{n+1} \circ l-H O M$
$\nsubseteq \quad$ sl-DT $T^{n} \circ T$ and
(2) $s l-D T^{n+2}$
$\not s l-D T^{n} \circ D T$.

\section{Proof.}

(1) Let $L \in \operatorname{dom}\left(s l-D T^{n+1}\right)-\operatorname{dom}\left(s l-D T^{n}\right)$ (such an $L$ exists by (1) of Theorem 3.1.3). Define $\tau$ as in Lemma 4.3.3. It is an easy exercise to show that $\tau \in s l-D T^{n+1} \circ l-H O M$. Suppose $\tau \in s l-D T^{n} \circ D T$. Then, by Lemma 4.3.3, $L \in \operatorname{dom}\left(s l-D T^{m}\right)$ holds for some $1 \leq m \leq n$, which is a contradiction. We have that $\tau \notin s l-D T^{n} \circ D T$.

(2) Since $l-H O M \subseteq s l-D T$ (see Corollary 2.1.2), the statement follows from (1) immediately.

The next technical lemma shows that an l-dt tree transformation exists, which cannot be induced by a sequence of sl-dt tree transducers followed by an nd-dt tree transducer.

Lemma 4.3.5 $l-D T \nsubseteq \& s l-D T^{*}$ o nd-DT

Proof. Let $\Sigma=\left\{\sigma^{(2)}, \#^{(0)}\right\}$. Define the 1-dt tree transducer

$$
T=\left(\left\{q_{0}, q_{1}, q_{2}\right\}, \Sigma,\left\{\#^{(0)}\right\}, q_{0}, R\right),
$$

where $R$ consist of the rules

- $q_{0}\left(\sigma\left(x_{1}, x_{2}\right)\right) \rightarrow q_{1}\left(x_{1}\right)$,

- $q_{1}\left(\sigma\left(x_{1}, x_{2}\right)\right) \rightarrow q_{2}\left(x_{1}\right)$,

- $q_{2}\left(\sigma\left(x_{1}, x_{2}\right)\right) \rightarrow q_{0}\left(x_{2}\right)$, and

- $\left.q_{0}(\#) \rightarrow \#\right\}$.

Let us investigate the set $\operatorname{dom}\left(\tau_{T}\right)$. (Since the output ranked alphabet of $T$ is $\left\{\#^{(0)}\right\}$, one can guess that the proof is actually concerned with domains.)

Define the set $H \subseteq \hat{T}_{\Sigma, 1}$ of trees as

$$
H=\left\{\sigma\left(\sigma\left(\sigma\left(t_{1}, x_{1}\right), t_{2}\right), t_{3}\right) \mid t_{1}, t_{2}, t_{3} \in T_{\Sigma}\right\} .
$$

It is easy to check that

$$
\operatorname{dom}\left(\tau_{T}\right)=\left\{h_{1}\left[\ldots h_{n}[\#] \ldots\right] \mid n \geq 0, h_{1}, \ldots, h_{n} \in H\right\} .
$$

Informally speaking, starting from $q_{0}, T$ steps to the left twice and to the right once on $\sigma \mathrm{s}$, and reaches $q_{0}$ again. Moreover, $T$ accepts \# also starting in state $q_{0}$. The tree transducer rejects every other tree, which is not in $H$. 
We show that $\tau_{T} \notin s l-D T^{*}$ o nd-DT, which implies the lemma immediately. To prove this, suppose the contrary, i.e. that there exist sl-dt tree transducers $T_{1}, \ldots, T_{n}$ and an nd-dt tree transducer $T_{n+1}$ such that $\tau_{T}=\tau_{T_{1}} \circ \ldots \circ \tau_{T_{n}} \circ \tau_{T_{n+1}}$. We abbreviate the right-hand side of the previous equation by $\tau$.

Suppose that $n$ is minimal. By (2) of Lemma 4.3.1, it can be assumed that the tree transducers $T_{1}, \ldots, T_{n}$ are op-ni-sl-dt.

We observe that $T_{n+1}$ is nondeleting and that, obviously, the tree transformation $\tau_{T}$ cannot be induced without deleting capacity. Hence $n \geq 1$ holds.

Let us assume $T_{1}=\left(Q_{1}, \Sigma, \Delta, p, R_{1}\right)$. (The input alphabet of $T_{1}$ can be supposed to be $\Sigma$ without loss of generality.)

Consider the trees in Figure 4.5. Since $\tau=\tau_{T}$ is supposed, it is easy to check that the following statement holds.

Statement. $t_{1}, t_{2} \in \operatorname{dom}(\tau), t_{3} \notin \operatorname{dom}(\tau)$

We investigate the rules of $T_{1}$. Considering Statement and that $T_{1}$ is nonincreasing, we have that there must be a rule of the form

$$
p(\#) \rightarrow \#_{1}
$$

in $R_{1}$, where $\#_{1} \in \Delta_{0}$.

By Statement, there must be a $(p, \sigma)$-rule in $R_{1}$. The tree transducer $T_{1}$ is op-ni-sl-dt, hence this rule is of one of the following forms:

(1) $p\left(\sigma\left(x_{1}, x_{2}\right)\right) \rightarrow \sigma_{1}\left(p^{\prime}\left(x_{1}\right)\right)$, where $\sigma_{1} \in \Delta_{1} \cup\left\{x_{1}\right\}$ and $p^{\prime} \in Q_{1}$. In this case, by Statement, it is easy to see that there must be a $\left(p^{\prime}, \sigma\right)$-rule in $R_{1}$. Moreover, $\operatorname{rhs}\left(p^{\prime}, \sigma\right)$ must contain $x_{1}$, otherwise $t_{2} \in \operatorname{dom}(\tau)$ would imply $t_{3} \in \operatorname{dom}(\tau)$, which contradicts Statement. By the sl property of $T_{1}$, it is possible if and only if $p^{\prime}=p$. However, in this case $t_{2} \in \operatorname{dom}(\tau)$ also implies $t_{3} \in \operatorname{dom}(\tau)$. We have that this form is not acceptable for the $(p, \sigma)$-rule.

(2) $p\left(\sigma\left(x_{1}, x_{2}\right)\right) \rightarrow \sigma_{1}\left(p^{\prime}\left(x_{2}\right)\right)$, where $\sigma_{1} \in \Delta_{1} \cup\left\{x_{1}\right\}$ and $p^{\prime} \in Q_{1}$. In this case $t_{2} \in \operatorname{dom}(\tau)$ implies $t_{3} \in \operatorname{dom}(\tau)$, hence this form contradicts Statement as well.

(3) $p\left(\sigma\left(x_{1}, x_{2}\right)\right) \rightarrow \sigma_{1}\left(p^{\prime}\left(x_{1}\right), p^{\prime \prime}\left(x_{2}\right)\right)$, where $\sigma_{1} \in \Delta_{2}$ and $p^{\prime}, p^{\prime \prime} \in Q_{1}$. We have that this form is the only possible form of $(p, \sigma)$.

Suppose that $p^{\prime} \neq p$ in (3). Then, by Statement, there must be a $\left(p^{\prime}, \sigma\right)$-rule in $R_{1}$. By the superlinear property of $T_{1}, \operatorname{rhs}\left(p^{\prime}, \sigma\right)$ must be a ground tree. However, in this case $t_{2} \in \operatorname{dom}(\tau)$ implies $t_{3} \in \operatorname{dom}(\tau)$, which contradicts Statement. We have obtained $p^{\prime}=p$.

Now suppose $p^{\prime}=p$ and $p^{\prime \prime} \neq p$. Similarly to the previous observations, one can easily conclude that a $\left(p^{\prime \prime}, \sigma\right)$-rule must be in $R_{1}$ and $\operatorname{rhs}\left(p^{\prime \prime}, \sigma\right)$ must be a ground tree. But in this case $t_{3} \in \operatorname{dom}(\tau)$ follows contradicting Statement. 


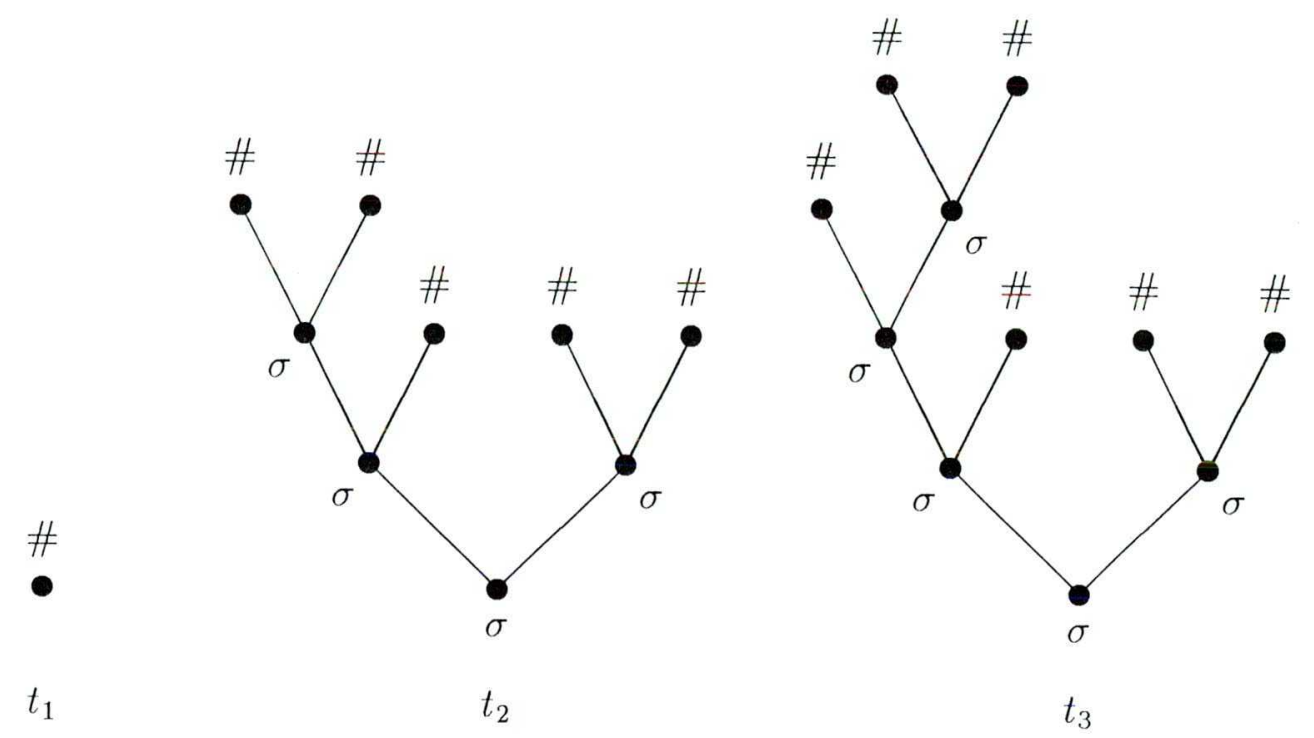

Figure 4.5: Example trees for Lemma 4.3.5 
Summarizing up, we have obtained that

$$
p\left(\sigma\left(x_{1}, x_{2}\right)\right) \rightarrow \sigma_{1}\left(p\left(x_{1}\right), p\left(x_{2}\right)\right) \in R_{1} .
$$

By the rules $(*)$ and $(* *)$, it can be supposed that there are no other rules in $R_{1}$. Moreover, $Q_{1}=\{p\}$ and $\Delta=\left\{\sigma_{1}^{(2)}, \#_{1}^{(0)}\right\}$ hold.

We have that $T_{1}$ is total and nonreducing, that is t-nr-op-ni-sl-dt tree transducer. Hence, $\tau_{T}=\tau_{T_{1}} \circ \ldots \circ \tau_{T_{n}} \circ \tau_{T_{n+1}}$ implies

$$
\tau_{T} \in t-n r-o p-n i-n d-s l-D T \text { o op-ni-sl-DT } T^{n-1} \circ n d-D T,
$$

for some $n \geq 1$.

Assume $n=1$, then $\tau_{T} \in t$-op-ni-nr-nd-sl-DT ond-DT $=n d-D T$ holds, which is obviously not true.

Assume $n>1$, then, by (7) of Corollary 2.1.12, $\tau_{T} \in$ op-ni-sl-DT $T^{n-1}$ ond-DT follows, which contradicts the minimality of $n$.

We have that suitable tree transducers $T_{1}, \ldots, T_{n+1}$ cannot exist.

Corollary 4.3.6 l-DT o nd-DT $\nsubseteq s l-D T^{*}$ o $n d-D T$

Now we begin to prove Lemma 4.2.3, which states that the diagram depicted in Figure 4.2 is the inclusion diagram of $|N F|$. First we show that all the six hierarchies appearing in $|N F|$ are proper.

Let $H$ be a set of tree transformation classes defined as

$$
H=\{I, l-D T, n d-D T, H O M, l-D T \circ n d-D T, D T\} .
$$

Observe that the hierarchies in $|N F|$ are of the form $\left\{s l-D T^{n} \circ X \mid n \geq 0\right\}$, where $X \in H$. We prove the following.

Lemma 4.3.7 Let $X \in H$ be arbitrary. Then $\left\{s l-D T^{n} \circ X \mid n \geq 0\right\}$ is a proper hierarchy.

Proof. Let $n \geq 0$ and $X \in H$. Recall sl-DT $T^{n+2} \not \subset s l-D T^{n} \circ D T$ from (2) of Corollary 4.3.4. Since $X \subseteq D T$, we get $s l-D T^{n+2} X \subseteq$ s $s l-D T^{n} \circ X$.

On the other hand, sl-DT $T^{n} \circ \subseteq s l-D T^{n+2} \circ X$ should be clear. Hence sl- $D T^{n} \circ X \subset$ sl-DT $T^{n+2} \circ X$ holds.

Now suppose that $s l-D T^{n} \circ X=s l-D T^{n+1} \circ X$. Then $s l-D T^{n+1} \circ X=$ $s l-D T^{n+2} \circ X$ also holds, which implies $s l-D T^{n} \circ X=s l-D T^{n+2} \circ X$. However, this contradicts the result of the previous paragraph.

We have $s l-D T^{n} \circ X \subset$ sl- $D T^{n+1} \circ X$, for every $n \geq 0$ and $X \in H$.

Let $X \in H$ and consider the classes $s l-D T^{*} \circ X=\bigcup_{n \geq 0}\left(s l-D T^{n} \circ X\right)$, which are the suprema of the corresponding hierarchies. Note that, for every $n \geq 0$, sl-DT $T^{n} \circ X \subset s l-D T^{*} \circ X$ holds by Lemma 4.3.7. 
Although the suprema are not elements of $|N F|$, they are very useful to prove certain inclusions in $|N F|$. Moreover, they make the inclusion diagram of $|N F|$ more complete and clear. Therefore, we represented them in the diagram.

In the following lemma we prove the inclusion relations between the suprema of the hierarchies.

Lemma 4.3.8 The diagram in Figure 4.6 is the inclusion diagram of the set

$$
\left\{s l-D T^{*} \circ X \mid X \in H\right\}
$$

i.e. of the set of suprema of the hierarchies in $|N F|$.

Proof. Observe that all inclusions depicted in Figure 4.6 are obvious, except

$$
\text { sl-DT* } T^{*} H O M \subseteq s l-D T^{*} \circ n d-D T
$$

and

$$
\text { sl-DT } T^{*} \text { o } l-D T \text { o } n d-D T \subseteq s l-D T^{*} \circ D T .
$$

Hence, to prove the lemma, it is enough to show that the following statements hold:

(1) $s l-D T^{*}$ o $l-D T \nsubseteq s l-D T^{*}$ o $n d-D T$

(2) $s l-D T^{*} \circ H O M \nsubseteq s l-D T^{*} \circ l-D T$

(3) $s l-D T^{*} \circ H O M \subset s l-D T^{*}$ o $n d-D T$

(4) $s l-D T^{*} \circ l-D T \circ n d-D T \subset s l-D T^{*} \circ D T$

We can prove these statements as follows:

(1) This follows from Lemma 4.3.5 immediately.

(2) Recall $H O M \nsubseteq l-D T^{2}$ from Figure 2 of [FülVág3], hence $s l-D T^{*} \circ H O M \nsubseteq$ $l-D T^{2}$. Since

$$
s l-D T^{*} \circ l-D T \subseteq l-D T^{2} \circ l-D T=l-D T^{2}
$$

(see (2) of Corollary 2.1.14 and Table 2 of [FülVág1]), the statement holds.

(3) Recall that $H O M=l-H O M \circ n d-H O M$ (see (29) in paper [FülVág1]). Since $l-H O M \subseteq s l-D T$ holds by Corollary 2.1 .2 and $n d-H O M \subseteq n d-D T$ is obvious, we have

$$
s l-D T^{*} \circ H O M \subseteq s l-D T^{*} \circ s l-D T \circ n d-D T=s l-D T^{*} \circ n d-D T .
$$

The hom tree transducers are total, which implies

$$
\operatorname{dom}\left(s l-D T^{*} \circ H O M\right)=\operatorname{dom}\left(s l-D T^{*}\right) \subset D R E C
$$




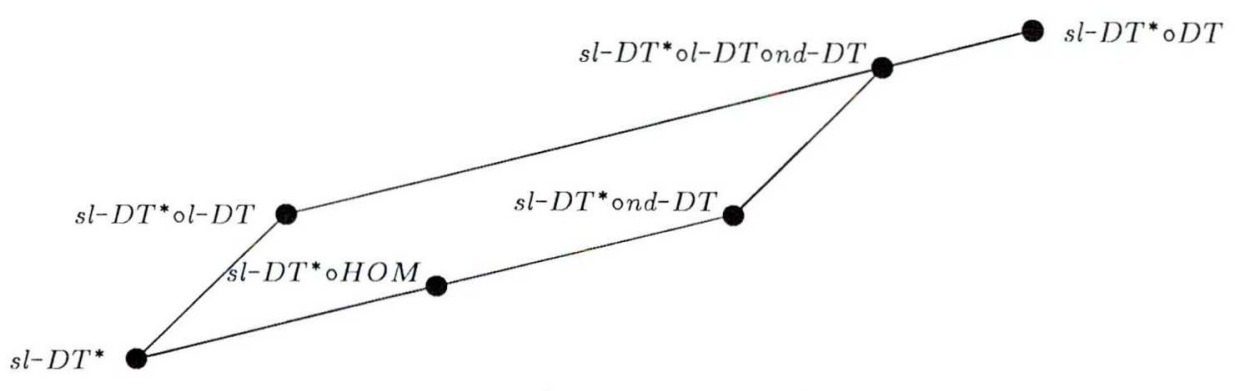

Figure 4.6: The inclusion diagram of suprema 
(see Theorem 4.3.2). On the other hand, $\operatorname{dom}(n d-D T)=D R E C$ implies

$$
\operatorname{dom}\left(s l-D T^{*} \text { o } n d-D T\right)=D R E C,
$$

hence the proper inclusion holds.

(4) Since $l-D T \circ n d-D T \subseteq D T$ (see Proposition 1.4.1),

$$
\text { sl-DT* ol-DT o } n d-D T \subseteq s l-D T^{*} \circ D T
$$

holds. Moreover,

$$
s l-D T^{*} \circ l-D T \circ n d-D T \subseteq l-D T^{2} \circ l-D T \circ n d-D T \subseteq l-D T^{2} \circ n d-D T
$$

(see (2) of Corollary 2.1.14 and Table 2 of [FülVág1]), and $D T \nsubseteq l-D T^{2}$ o nd-DT (see Figure 2 of [FülVág6]), hence the inclusion is proper.

Observe that the inclusion relation between any two elements depicted in Figure 4.6 can be determined using statements (1)-(4).

For example, we show sl-DT* $\subset$ sl-DT* o $l-D T$. The inclusions $s l-D T^{*} \subseteq$ $s l-D T^{*} \circ l-D T$ and $s l-D T^{*} \subseteq s l-D T^{*} \circ n d-D T$ should be obvious. Then, considering (1), we have the desired result immediately.

Besides the hierarchies, there are the classes $l-D T^{2}, l-D T \circ H O M, l-D T^{2}$ 。 $n d-D T$ and $D T^{2}$ in $|N F|$. In the following lemma we attach them to the inclusion diagram of the suprema of the hierarchies.

Lemma 4.3.9 The diagram in Figure 4.7 is the inclusion diagram of the set consisting of $l-D T^{2}, l-D T \circ H O M, l-D T^{2} \circ n d-D T, D T^{2}$, and the suprema of the six hierarchies.

Proof. The inclusion relations between the suprema of the hierarchies are clear by Lemma 4.3.8.

In [FülVág6] it has been proved that the four new classes obey the following inclusion relations (see Figure 2 in that paper):

$$
l-D T^{2} \subset l-D T \circ H O M \subset l-D T^{2} \circ n d-D T \subset D T^{2} .
$$

First we show that none of the suprema (hence none of the elements of the hierarchies) includes any of the new classes. To prove this, it is enough to show that the least new element is not included in the largest supremum, i.e.

$$
l-D T^{2} \not \subset s l-D T^{*} \circ D T
$$

holds. 


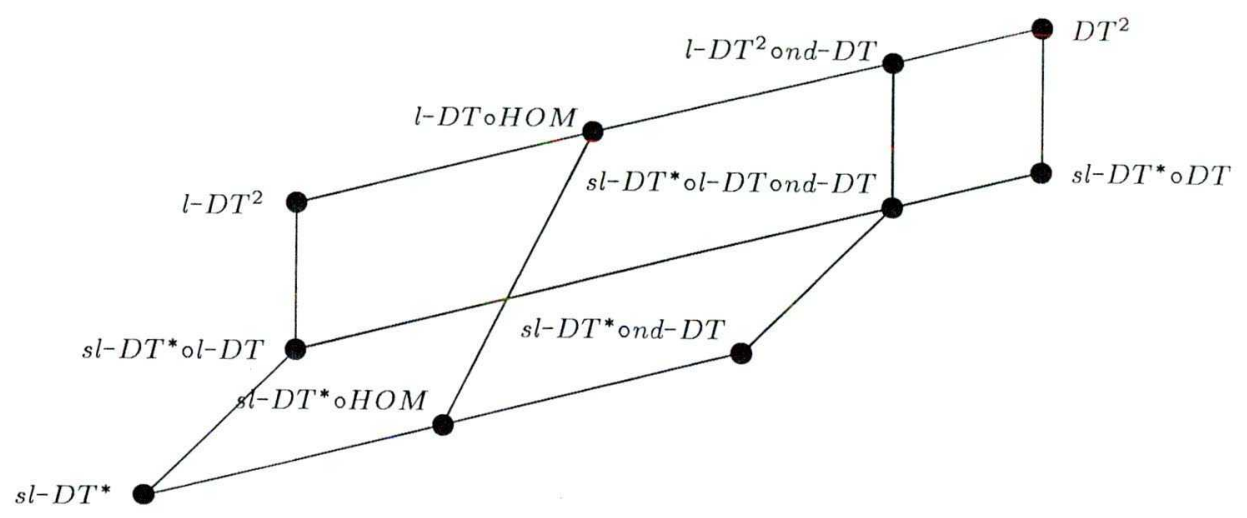

Figure 4.7: The inclusion diagram of suprema and top elements 
By Theorem 4.3.2, there exists a tree language $L$ such that $L \in D R E C-$ $\operatorname{dom}\left(s l-D T^{*}\right)$. Construct $\tau$ as defined in Lemma 4.3.3. Then it should be clear that $\tau \notin s l-D T^{n} \circ D T$ for every $n \geq 0$, hence $\tau \notin s l-D T^{*} \circ D T$.

On the other hand, $L \in D R E C=\operatorname{dom}(l-D T)$, therefore $\tau \in l-D T^{2}$ should be obvious.

Now we prove that the new elements are the topmost elements in the inclusion diagram of $|N F|$. We define the set $G$ of tree transformation classes as

$$
G=\{l-D T, H O M, l-D T \circ n d-D T, D T\} .
$$

Note that the set of new four elements of $|N F|$ is exactly $\{l-D T \circ X \mid X \in G\}$ (for $l-D T$ o $D T=D T^{2}$ see Table 2 of [FülVág6]).

Let $X \in G$ be arbitrary. Observe that $l-D T^{2} \circ X=l$-DT $\circ X$ holds (see Table 2 of [FülVág6]). This implies the inclusion sl-DT* $X \subseteq l-D T \circ X$, because sl-DT* $\circ \subseteq \subseteq l-D T^{2} \circ X=l-D T \circ X$ (see (2) of Corollary 2.1.14). Moreover, the inclusion must be proper by $(*)$, that is

$$
s l-D T^{*} \circ X \subset l-D T \circ X
$$

holds, for each $X \in G$.

Finally, we state that there are no other edges corresponding to the topmost elements in the inclusion diagram of $|N F|$, besides the ones depicted in Figure 4.7. To show this, it is enough to prove the following statements:

(1) $H O M \nsubseteq l-D T^{2}$

(2) $n d-D T \nsubseteq l-D T \circ H O M$

(3) $D T \nsubseteq l-D T^{2}$ ond-DT

For example, we can show that $s l-D T^{*}$ o $n d-D T \nsubseteq l-D T^{2}$. For if $s l-D T^{*}$ 。 $n d-D T \subseteq l-D T^{2}$ holds, then, by HOM $\subseteq$ sl-DT* $\circ H O M$ and $s l-D T^{*} \circ H O M \subseteq$ $s l-D T^{*} \circ n d-D T$, we get $H O M \subseteq l-D T^{2}$, which contradicts (1).

However, (1), (2), and (3) have already been proved in [FülVág6] (see Figure 2 in that paper). With this, we have proved Lemma 4.3.9.

We should still prove the inclusions between the elements of the hierarchies. The following corollary shows that, roughly speaking, there can only be edges descending from right to left in the inclusion diagram of $|N F|$.

Corollary 4.3.10 Denote the bottom elements of the hierarchies as $X_{1}=I, X_{2}=$ $l$-DT, $X_{3}=H O M, X_{4}=n d-D T, X_{5}=l-D T \circ n d-D T$ and $X_{6}=D T$. Let $i, j$ be arbitrary integers such that $1 \leq i<j \leq 6$. Then $X_{j} \nsubseteq$ sl-DT* $\circ X_{i}$ holds.

Proof. By statements (1), (2), and (3) in the proof of Lemma 4.3.9, we have most of these results immediately.

Only the following two cases should be checked: 
(1) $l-D T \nsubseteq s l-D T^{*}$ follows from Theorem 2.1.13.

(2) $l-D T \circ n d-D T \nsubseteq s l-D T^{*}$ o $n d-D T$ holds by Corollary 4.3.6.

Thus we are done.

Now we can finish the proof of Lemma 4.2.3.

Proof of Lemma 4.2.3. Recall that the inclusion relations between the topmost elements and the suprema of the six hierarchies have already been clarified (see Lemma 4.3.8).

First we prove that the inclusions depicted in Figure 4.2 hold. Let $n \geq 1$. All inclusions should be clear, except the following ones:

(1) $s l-D T^{n-1} \circ H O M \subseteq s l-D T^{n} \circ n d-D T$

(2) $s l-D T^{n-1} \circ \mathrm{l}-D T \circ n d-D T \subseteq s l-D T^{n-1} \circ D T$

We can prove these statements as follows.

(1) Recall the decomposition equation $H O M=l-H O M \circ n d-H O M$ (see (29) in [FülVág1]). Since $l-H O M \subseteq s l-D T$ holds by Corollary 2.1.2 and $n d-H O M \subseteq$ $n d-D T$ is obvious, we have sl-DT $T^{n-1} \circ H O M \subseteq s l-D T^{n-1} \circ$ sl-DT o nd-DT = sl-DT $T^{n}$ o nd-DT.

(2) The inclusion $l-D T \circ n d-D T \subseteq D T$ follows from Proposition 1.4.1.

Observe that, by Lemma 4.3.7 and Corollary 4.3.10, the inclusions depicted in Figure 4.2 are necessarily proper.

Finally, we show that there cannot be other inclusions. To prove this, it is enough to consider Corollary 4.3.10 and the following statements:

(3) $l-D T \nsubseteq s l-D T^{*}$ o $n d-D T$, by Lemma 4.3.5.

(4) sl-DT $T^{n} \circ H O M \nsubseteq s l-D T^{n-1} \circ D T$, by (1) of Corollary 4.3.4.

(5) $s l-D T^{n+1} \nsubseteq £ s l-D T^{n-1} \circ D T$, by (2) of Corollary 4.3.4.

Now we have obtained that the relations between any two elements depicted in Figure 4.2 can be determined using Corollary 4.3 .10 and the statements (1)-(5).

For example, we show

$$
\text { sl-DT } T^{3} \text { o } H O M \subset s l-D T^{7} \text { ond-DT. }
$$

It should be clear that

$$
s l-D T^{3} \circ H O M \subseteq s l-D T^{6} \circ H O M .
$$


By statement (1), we have

$$
\text { sl-DT } 6 \text { o HOM } \subset \text { sl-DT } T^{7} \text { ond-DT. }
$$

Hence

$$
s l-D T^{3} \circ H O M \subset s l-D T^{7} \circ n d-D T
$$

holds.

With this, we finished the proof of the Lemma 4.2.3. 


\section{Conclusions}

In this thesis we considered superlinear deterministic top-down tree transducers and the class sl-DT of superlinear deterministic top-down tree transformations. Our main results are as follows:

- The classes sl-DT and $t$-sl-DT are not closed under the composition.

- $t-l-D T-s l-D T^{+} \neq \emptyset$, where $s l-D T^{+}$is the transitive closure of the class sl-DT under the composition. Roughly speaking, even the consecutive application of arbitrary many sl-dt tree transducers has no enough transformational power to generate all l-dt tree transformations.

- $D T=n d-H O M \circ$ o sl-DT, that is sl-dt tree transducers have enough computational capacity to generate all $\mathrm{dt}$ tree transformations with the help of nondeleting homomorphism tree transducers.

- The class $\operatorname{dom}(s l-D T)$ is exactly su-DREC, i.e. the subclass of DREC consisting of those tree languages which are recognized by semi-universal deterministic top-down tree recognizers (su-dttr's).

- For any deterministic recognizable tree language $L$, it is decidable whether $L$ is in $\operatorname{dom}(s l-D T)$. Namely, $L$ is in $\operatorname{dom}(s l-D T)$ if and only if the minimal dttr recognizing $L$ is an su-dttr.

Thus, being the minimal dttr unique up to the isomorphism, the decision procedure is quite simple. Given a $\operatorname{dttr} T$ recognizing $L$, the $\operatorname{dttr} T$ is to be minimalized, then it is decidable by direct inspection whether the resulting minimal dttr is semi-universal.

- The class range $(s l-D T)$ is exactly $R E C$, that is the class of all recognizable tree languages.

- The hierarchies

$$
\begin{aligned}
& \quad\left\{\operatorname{dom}\left(s l-D T^{n}\right) \mid n \geq 0\right\}, \\
& \left\{s l-D T^{n} \mid n \geq 0\right\}, \text { and } \\
& \\
& \left\{t-s l-D T^{n} \mid n \geq 0\right\} \\
& \text { are proper. }
\end{aligned}
$$


- We have considered the monoid $[M]$ generated by the tree transformation classes $H O M$, sl-DT, $l-D T, n d-D T$, and $D T$ with the composition operation. This is the first work, where $s l-D T$ is taken as a generator element of a monoid of tree transformation classes.

Using string rewriting techniques, we have developed an algorithm which, given any two elements $X_{1} \circ X_{2} \circ \ldots \circ X_{m}$ and $Y_{1} \circ Y_{2} \circ \ldots \circ Y_{n}$ of $[M]$, can decide whether the inclusion $X_{1} \circ X_{2} \circ \ldots \circ X_{m} \subseteq Y_{1} \circ Y_{2} \circ \ldots \circ Y_{n}$ holds. Of course, in this case it is also decidable whether $\supseteq,=$, or incomparability holds. We have represented the elements of $[M]$ by strings, and have presented a terminating and confluent string rewriting system $R$ as well as the inclusion diagram of the normal forms with respect to $R$.

The inclusion between two elements of $[M]$ can be decided in the following way. We reduce the strings representing the tree transformation classes $X_{1} \circ X_{2} \circ \ldots \circ X_{m}$ and $Y_{1} \circ Y_{2} \circ \ldots \circ Y_{n}$ to normal forms with respect to $R$. The string rewriting system $R$ is constructed in such a way that $\subseteq$ (resp. $\supseteq$, =, incomparability) holds between the two tree transformation classes if and only if the same relation holds between the tree transformation classes represented by the corresponding normal forms. However, this latter can be read from the inclusion diagram depicted in Figure 4.2.

Finally, we arise two open problems, which may be topic of further research. These are as follows:

Open Problem 1 The superlinear property could easily be defined also for deterministic bottom-up tree transformations, as, e.g., the linearity is defined as well. It would be an interesting task to characterize superlinear deterministic bottom-up tree transducers with respect to similar principles as it done concerning the top-down version in this work.

Open Problem 2 It is known that the equivalence problem of deterministic topdown tree transducers is decidable, see [Ési1]. However, the undecidability of the equivalence problem of nondeterministic top-down tree transducers immediately follows from the fact that the equivalence problem of GSM's is undecidable, see [Gri]. Moreover, this latter result also implies that even the equivalence problem of linear nondeterministic top-down tree transducers is undecidable.

The concept of superlinearity can be generalized to nondeterministic topdown tree transducers in a natural way. It is easy to see that the undecidability of the equivalence problem of superlinear nondeterministic top-down tree transducers does not follow (at least immediately not) from the result of [Gri] on GSM's. Thus decidability questions concerning superlinear nondeterministic top-down tree transducers are worth to be studied. Actually, we guess that the equivalence problem of superlinear nondeterministic topdown transducers is decidable. 
Up to now, besides the class of deterministic top-down tree transducers, the equivalence problem has been shown to be decidable only for one subclass of nondeterministic top-down transducers, namely for the class of linear strict letter to-letter top-down tree transducers, see [AndBos]. Note that those tree transducers are the same as our ni-nr-1 top-down tree transducers. 


\section{Összefoglalás (Summary in Hungarian)}

A fatranszfomátorok tulajdonságait a hetvenes évek elejétől kutatják az elméleti számítástudományon belül. Ezek olyan véges eszközök, amelyek rangolt ábécék feletti termeket, más néven fákat képesek feldogozni. Egy fatranszformátor egy fahalmazok feletti bináris relációt, ú.n. fatranszformációt indukál.

A kutatások során számos fatranszformátor tipust vizsgáltak. A top-down fatranszformátor fogalmát Rounds ([Rou]) és Thatcher ([Tha1]) vezette be, a bottom-up változatot pedig Thatcher ([Tha2]). Később a transzformációs kapacitás növelése érdekében bonyolultabb tipusokat is definiáltak, nevezetesen Engelfriet a regulárisan elörenézö top-down fatranszformátort ([Eng2]), Engelfriet és Vogler a makró ([EngVog1]), a high-level ([EngVog2]) és a moduláris ([EngVog3]) fatranszformátort, Fülöp az attribútumos fatranszformátort ([Fül1]), valamint Vogler a high-level moduláris fatranszformátort.

Ebben a dolgozatban csak determinisztikus top-down fatranszformátorokat, ill. ezek által indukált fatranszformációkat vizsgúlunk.

A top-down fatranszformátorok vizsgálatának gyakorlati motivációját az adja, hogy a szintaxis vezérelt fordítóprogramok matematikai modelljeként használhatók (ld. [Eng4]).

A determinisztikus top-down fatranszformátoroknak számos altipusát definiálták. Ebben a disszertációban foglalkozunk többek között lineáris, nemtörlö és homomorfizmus determinisztikus top-down fatranszformátorokkal.

A dolgozatban fatranszformáció osztályon általában meghatározott tipusú fatranszformátorok osztálya által indukált fatranszformációk osztályát értjük. Eszerint tehát definiálhatjuk a determinisztikus top-down fatranszformációk osztályát $(D T)$, valamint annak totális $(t-D T)$, lineáris $(l-D T)$, nemtörlö ( $n d-D T)$ és homomorfizmus $(H O M)$ részosztályait. A tipusok szabadon kombinálhatók, így még speciálisabb fatranszformáció osztályok hozhatók létre, például a lineáris nemtörlő fatranszformációk osztálya $(l-n d-D T)$.

Adott tipusú fatranszformátorokat vizsgálva mindig felmerül a kérdés, hogy milyen input fahalmazokat képesek feldolgozni és milyen output fahalmazok jöhetnek létre ezen feldolgozások eredményeként. Egy fatranszformátor lehetséges input, illetve output fahalmazait az általa indukált fatranszfromáció értelmezési 
tartományának (domain) és értékkészletének (range) hívjuk.

A fahalmazokat fanyelveknek is nevezzük. Hasonlóan a string nyelvekhez, a fanyelvekre is léteznek véges felismerö eszközök (ld. [GécSte4]), amelyek segítségével definiálhatjuk a felismerhetö, illetve a determinisztikusan felismerhetö fanyelvek osztályait. Kiderült, hogy a determinisztikus top-down fatranszformációk értelmezési tartományai éppen a determinisztikusan felismerhetö fanyelvek, továbbá, hogy a lineáris determinisztikus top-down fatranszformációk értékkészleteinek osztálya azonos a felismerhető fanyelvek osztályával.

Mivel a fatranszformációk bináris relációk, a fatranszformációk és fatranszformáció osztályok kompozíciója (jelölése: o) jól definiált. Ugyanakkor a gyakorlati motiváció szempontjából is igen fontos mind a kompozíció, mind a dekompozíció. A kompozíció tanulmányozásával megtudhatjuk, hogy adott tipusú fatranszformátorokkal végzett többlépcsős fordítás helyettesíthetö-e egyetlen fatranszformátor alkalmazásával. A dekompozíció pedig azt mutatja meg, hogy egy adott tipusú fatranszformátor által indukált fordítás elvégezhetö-e valamely egyszerübb tipusok többlépcsős alkalmazásával.

A top-down fatranszformátorokat és fatranszformációkat igen intenzíven tanulmányozták. Az alábbiakban vázlatosan bemutatjuk, hogy milyen irányú kutatások folytak és milyen eredmények születtek ezen a területen.

Úttörő jellegü kutatásaik során Rounds ([Rou]), Thatcher ([Tha1]), Engelfriet ([Eng1], [Eng3]) és Baker ([Bak1], [Bak2],[Bak3]) számos altipust definiáltak (például lineáris, nemtörlö, stb.). Vizsgálták ezek egymáshoz viszonyított transzformációs képességeit, továbbá a kapcsolódó fatranszformáció osztályok néhány zártsági tulajdonságát is megmutatták. Ezen eredményeknek összefoglalása megtalálható [GécSte4]-ben.

A top-down fatranszformációk értékkészleteinek és értelmezési tartományainak felismerhetösége számos kutatót foglalkoztatott, ld. [Rou], [Eng2], [GécSte4], [FülVág1] és [FülVág3].

A top-down fatranszformátorok ekvivalenciájának eldönthetetlensége az általános esetben azonnal adódik Griffiths ([Gri]) eredményéböl, mely szerint az általánosított szekvenciális gépek (GSM) ekvivalenciája nem eldönthetö. Kiderült azonban, hogy a determinisztikus esetben már eldönthetö az ekvivalencia, ld. [Ési1] és [Zac]. Az ekvivalencia eldönthetöségének problémáját néhány egyéb speciális nemdeterminisztikus tipusra is megvizsgálták ([AndBos]). Továbbá, egyéb eldönthetőségi kérdések is tanulmányozásra kerültek (injektivitás, az értékkészlet felismerhetősége, stb.), ld. [Ési1], [Ési2], [Fül4] és [FülGye].

A fatranszformáció osztályok kompozíciós és dekompozíciós tulajdonságainak kutatása igen gyümölcsözö területnek bizonyult. Szinte minden fatranszformátorokkal foglakozó publikáció tartalmaz ilyen eredményt, így kompozíciós és dekompozíciós egyenletek egy igen bö készlete áll rendelkezésre. Ezek kezelhetősége érdekében kívánatossá vált egy egységes megközelítési mód kidolgozása az ilyen jellegü kutatásokra vonatkozóan. Fülöp és Vágvölgyi ([FülVág4], [FülVág6]) javasolt egy általános módszert olyan algoritmus kifejlesztésére, amely fatransz- 
formáció osztályok egy tetszőlegesen rögzített halmaza (bázis halmaz) esetén el tudja dönteni, hogy milyen tartalmazási viszony áll fent a bázis halmaz elemeiből kompozícióval kapott fatranszformáció osztályok között. Az eljárásnak számos alkalmazása ismert, ld. [FülVág4], [FülVág5], [Fül2], [SluVág] és [GyeVág].

Megjegyezzük, hogy magyar nyelven is hozzáférhetö néhány fatranszformátorokkal kapcsolatos publikáció, ld. [GécSte1], [GécSte2] és [Fül3].

Jelen dolgozat tárgya a determinisztikus top-down fatranszformátorok egy új tipusának, a szuperlineáris fatranszformátoroknak a vizsgálata. A szuperlineáris determinisztikus top-down fatranszformációk osztályát sl-DT-vel jelöljük, A szuperlineáris determinisztikus top-down fatranszformátorok speciális lineáris determinisztikus top-down fatranszformátorok, továbbá sl-DT $\subset l-D T$ is teljesül.

A szuperlineáris determinisztikus top-down fatranszformátorok bevezetését Heiko Vogler javasolta 1992-ben egy Fülöp Zoltánnal folytatott megbeszélés során. A motivációt a jól ismert $D T=n d-H O M \circ l-D T$ dekompozíciós egyenlet adta, amely elöször [Eng1]-ben, illetve [Bak3]-ban jelent meg. Sejtésük szerint a fenti egyenletben $l-D T$ helyettesíthetö a $D T$ osztály egy szükebb részosztályával is, nevezetesen a szuperlineáris determinisztikus top-down fa-transzformációk osztályával $(s l-D T)$.

A dolgozatban megvizsgáljuk a szuperlineáris determinisztikus top-down fatranszformátorok, ill. a kapcsolódó sl-DT fatranszformáció osztály tulajdonságait. Bár a kutatás kiindulópontja a $D T=n d-H O M \circ s l-D T$ dekompozíciós egyenlet volt, a szuperlineáris determinisztikus top-down fatranszformátorok és fatranszformációk tanulmányozása számos egyéb érdekes eredményt is hozott. A dolgozatban felvetett és megoldott problémák motivációját többnyire a korábbi, fatranszformátorokkal foglalkozó munkák adták, ld. például [Eng1], [Eng3], [Bak3], [FülVág1] és [FülVág2]. A fóbb kérdések a következők voltak:

- Milyen tartalmazási relációban áll az sl-DT osztály a már ismert determinisztikus top-down fatranszformáció osztályokkal, mint például DT-vel, vagy $l$-DT-vel? Másképpen megfogalmazva, hogyan hasonlítható a szuperlineáris determinisztikus top-down fatranszformátorok fordítási képessége a már ismert tipusokéhoz?

- Zárt-e az sl-DT osztály a kompozícióra?

- Milyen tipusú fanyelvek lehetnek értelmezési tartományai, illetve értékkészletei szuperlineáris determinisztikus top-down fatranszformációknak?

- Hogyan jellemezhetjük az sl-DT osztály más ismert fatranszformáció osztályokkal képezett kompozícióit?

A kutatás során olyan eredmények is jelentkeztek, amelyek önmagukban, a szuperlineáris determinisztikus top-down fatranszformátoroktól eltekintve is 
érdekesek lehetnek. Ilyen például a determinisztikus top-down fafelismerök minimalizálási algoritmusa (ld. az 1.4.4 alfejezetben), a szemiuniverzális determinisztikus top-down fafelismerö definíciója, valamint az a tény, hogy a minimalizálás megőrzi a szemiuniverzális tulajdonságot (ld. a 2.2 fejezetben), továbbá a $D T=$ op-ni-DT o nr-l-nd-HOM dekompozíciós egyenlet (ld. Lemma 2.1.9).

Megjegyezzük, hogy a dolgozatban leírtak már korábban publikálásra kerül, ld. [DánFül1], [DánFül2] és [Dán].

A dolgozatban található fontosabb eredmények a következők:

- Az sl-DT és t-sl-DT fatranszformáció osztályok nem zártak a kompozícióra.

- $t-l-D T-s l-D T^{+} \neq \emptyset$, ahol sl-DT $T^{+}$az sl-DT osztálynak a kompozícióra vonatkozó tranzitív lezártja. Eszerint tehát létezik olyan lineáris determinisztikus fatranszformáció, amelyet nem lehet indukálni szuperlineáris determinisztikus fatranszformátorok semmilyen véges sorozatával.

- $D T=n d-H O M \circ s l-D T$, azaz egy tetszőleges determinisztikus top-down fatranszformáció mindig indukálható egy nemtörlő homomorfizmus top-down fatranszformátor és egy szuperlineáris determinisztikus top-down fatranszformátor egymás utáni alkalmazásával.

- A szuperlineáris determinisztikus fatranszformációk értékkészleteinek osztálya $(\operatorname{dom}(s l-D T))$ éppen a szemiuniverzális determinisztikus top-down fafelismerök (dttr-ek) által felismert fanyelvek osztálya (su-DREC).

- Eldönthető, hogy egy tetszőleges $L$ determinisztikusan felismerhetö fanyelv eleme-e a dom(sl-DT) fanyelv osztálynak. Nevezetesen, $L$ pontosan akkor eleme a dom(sl-DT) osztálynak, ha az $L$ fanyelvet felismerö minimális dttr szemiuniverzális.

Mivel a minimális dttr az izomorfizmustól erejéig egyértelmü, az eldöntési algoritmus a következö. Meg kell adni egy tetszőleges, $L$-t felismerő dttrt, amelyet minimalizálva a kapott minimális dttr-röl a szabályai alapján egyszerü számolással eldönthetö, hogy szemiuniverzális-e.

- A range $(s l-D T)$ fanyelv osztály azonos a felismerhetö fanyelvek osztályával $(R E C)$.

- Az alábbi hierarchiák valódiak:

$$
\begin{aligned}
& \left\{\operatorname{dom}\left(s l-D T^{n}\right) \mid n \geq 0\right\}, \\
& \left\{s l-D T^{n} \mid n \geq 0\right\} \text { és } \\
& \left\{t-s l-D T^{n} \mid n \geq 0\right\} .
\end{aligned}
$$

- Megvizsgáltuk a $H O M$, sl-DT, $l-D T, n d-D T$ és $D T$ fatranszformáció osztályok, mint generátor elemek által a kompozícióval generált $[M]$ monoidot. 
A string átíró rendszerek területéröl vett technikák alkalmazásával kifejlesztettünk egy olyan algoritmust, amely tetszőleges $[M]$-beli $X_{1} \circ X_{2} \circ \ldots \circ$ $X_{m}$ és $Y_{1} \circ Y_{2} \circ \ldots \circ Y_{n}$ elemek esetén eldönti, hogy az $X_{1} \circ X_{2} \circ \ldots \circ X_{m} \subseteq$ $Y_{1} \circ Y_{2} \circ \ldots \circ Y_{n}$ tartalmazás teljesül-e. (Ekkor nyilván az is eldönthetö, hogy $\supseteq,=$, vagy esetleg összehasonlíthatatlanság áll-e fent közöttük.)

$\mathrm{Az}[M]$ elemeit stringekkel reprezentáljuk és megadunk egy $R$ konfluens és termináló string átíró rendszert, amelyben az átírási szabályokat kompozíciós és dekompozíciós egyenletekből származtatjuk. Megadjuk továbbá az $R$-normálformák által reprezentált fatranszformáció osztályok tartalmazási diagramját.

A tartalmazási reláció két fenti alakú $[M]$-beli elem esetén ekkor a következö módon dönthető el. Normálformává redukáljuk $R$ szerint az $X_{1} \circ X_{2} \circ \ldots \circ X_{m}$ és az $Y_{1} \circ Y_{2} \circ \ldots \circ Y_{n}$ osztályokat reprezentáló stringeket. Az $R$ string átíró rendszer konstrukciójából adódóan a két fatranszformáció osztály között pontosan akkor teljesül $\subseteq$ (illetve $\supseteq$, =, vagy összehasonlíthatatlanság), ha ugyanezen reláció áll fent a normálformák által reprezentált osztályok között is. Ez utóbbi azonban könnyen leolvasható a normálformák tartalmazási diagramjáról (ld. Figure 4.2). 


\section{Bibliography}

[AndBos] Andre, Y. and Bossut, F., Decidability of equivalence for linear letter-to-letter top-down tree transducers, Proceedings of FCT '93 (Z. Ésik ed.), Lecture Notes in Computer Science 710 (1993) 142-151.

[Bak1] Baker, B. S., Tree transducers and families of tree languages, 5th Ann. ACM STC (1973), 200-206.

[Bak2] Baker, B. S., Tree transducers and tree languages, Information and Control 37 (1978), 241-266.

[Bak3] Baker, B. S., Composition of top-down and bottom-up tree transductions, Information and Control 41 (1979) 186-213.

[Boo] Book, R. V., Thue systems and the Church-Rosser Property: replacement systems, specification of formal languages and presentation of monoids, Progress in combinatorics on words (L. Cummings ed.), 1-38, Academic Press, 1983, New York

[BooOtt] Book, R. V. and Otto, F., String-rewriting systems, Springer-Verlag, 1993.

[BurSan] Burris, S. and Sankappanavar, H. P., A course in universal algebra, Springer-Verlag, 1981.

[Dán] Dányi, G., On domain and range tree languages of superlinear deterministic top-down tree transformations, Acta Cybernetica, 12 (1996) 216-277.

[DánFül1] Dányi, G. and Fülöp, Z., Superlinear deterministic top-down tree transducers, Mathematical Systems Theory 29 (1996) 507-534.

[DánFül2] Dányi, G. and Fülöp, Z., Compositions with superlinear deterministic top-down tree transducers, Theoretical Computer Science, accepted for publication.

[Eng1] Engelfriet, J., Bottom-up and top-down tree transformations - a comparison, Mathematical Systems Theory 9 (1975) 198-231. 
[Eng2] Engelfriet, J., Top-down tree transducers with regular look-ahead, Mathematical Systems Theory 10 (1977) 289-303.

[Eng3] Engelfriet, J., Three hierarchies of transducers, Mathematical Systems Theory 15 (1982) 95-125.

[Eng4] Engelfriet, J., Tree transducers and syntax-directed semantics, Proceedings of the 7th CAAP, Lille, 1982, 95-125.

[EngVog1] Engelfriet, J. and Vogler, H., Macro tree transducers, Journal of Computational System Sciences 31 (1985) 71-146.

[EngVog2] Engelfriet, J. and Vogler, H., High level tree transducers and iterated push-down tree transducers, Acta Informatica 26 (1988) 131-192.

[EngVog3] Engelfriet, J. and Vogler, H., Modular tree transducers, Theoretical Computer Science 78 (1991) 267-303.

[Ési1] Ésik, Z., Decidability results concerning tree transducers I., Acta Cybernetica 5 (1980) 1-20.

[Ési2] Ésik, Z., Decidability results concerning tree transducers II., Acta Cybernetica 6 (1983) 303-314.

[Fül1] Fülöp, Z., On attributed tree transducers, Acta Cybernetica 5 (1981) 261-279.

[Fül2] Fülöp, Z., A complete description for a monoid of deterministic bottom-up tree transformation classes, Theoretical Compututer Science 88 (1991) 253-268.

[Fül3] Fülöp Z., Eldönthetöségi kérdések fatranszformáció osztályok által generált monoidokban, Alkalmazott Matematikai Lapok 15 (199091), 219-266.

[Fül4] Fülöp, Z., Undecidable properties of deterministic top-down tree transducers, Theoretical Computer Science 134 (1994) 311-328.

[FülGye] Fülöp, Z. and Gyenizse, P., On injectivity of deterministic top-down tree transducers, Information Processing Letters 48 (1993), 183-188.

[FülVág1] Fülöp, Z. and Vágvölgyi, S., Results on compositions of deterministic root-to-frontier tree transformations, Acta Cybernetica 8 (1987) 4961.

[FülVág2] Fülöp, Z. and Vágvölgyi, S, An infinite hierarchy of tree transformations in the class NDR, Acta Cybernetica 8 (1987) 153-168. 
[FülVág3] Fülöp, Z. and Vágvölgyi, S., On domains of tree transducers, Bulletin of the EATCS 34 (1988) 55-61.

[FülVág4] Fülöp, Z. and Vágvölgyi, S., A finite presentation for a monoid of tree transformation classes, Proceedings of 2nd Conference on Automata, Languages, and Programming Systems (F. Gécseg and I. Peák Eds.), Salgótarján, 1988.

[FülVág5] Fülöp, Z. and Vágvölgyi, S., A complete classification of deterministic root-to-frontier tree transformation classes, Theoretical Compututer Science 81 (1991) 1-15.

[FülVág6] Fülöp, Z. and Vágvölgyi, S., Decidability of the inclusion in monoids generated by tree transformation classes, Tree Automata and Languages (M. Nivat and A. Podelski eds.), Elsevier Science Publishers B. V., Amsterdam, 1992, 381-408.

[GécSte1] Gécseg, F. and Steinby, M., A faautomaták algebrai elmélete I., Matematikai Lapok 26 (1978), 169-207.

[GécSte2] Gécseg, F. and Steinby, M., A faautomaták algebrai elmélete II., Matematikai Lapok 27 (1979), 283-366.

[GécSte3] Gécseg, F. and Steinby, M., Minimal ascending tree automata, Acta Cybernetica 4 (1980) 37-44.

[GécSte4] Gécseg, F. and Steinby, M., Tree automata, Akadémiai Kiadó, Budapest, 1984.

[Gri] Griffiths, T. V., The unsolvability of the equivalence problem for $\Lambda$ free nondeterministic generalized machines, Journal of the ACM 15 (1968) 409-413.

[GyeVág] Gyenizse, P. and Vágvölgyi, S., Compositions of deterministic bottom-Up, top-down, and regular look-ahead tree transformations, Theoretical Compututer Science 156 (1996) 71-97.

[Huet] Huet, G., Confluent Reductions: Abstract properties and applications to term rewriting systems, Journal of the Association for Computing Machinery 4 (1980) 797-821.

[Jan] Jantzen, M., Confluent string rewriting, Springer-Verlag, 1988.

[Rou] Rounds, W. C., Mappings on grammars and trees, Mathematical Systems Theory 4 (1970) 257-287. 
[SluVág] Slutzki, G. and Vágvölgyi, S., A hierarchy of deterministic top-down tree transformations, Proceedings of FCT '93 (Z. Ésik ed.), Lecture Notes in Computer Science 710 (1993) 440-451.

[Tha1] Thatcher, J. W., Generalized sequential machine maps, Journal of Computational System Sciences 4 (1970) 339-367.

[Tha2] Thatcher, J. W., Tree auttomata: an informal survey, Currents in the Theory of Computing (A. V. Aho ed.) Prenttice-Hall, 1973, 143172 .

[Vog] Vogler, H., High level modular tree transducers, 1978-1988, Ten years IIG, Institutes for Information Processing (H. J. Pongratz and W. Schinnerl eds.), Report Nr. 260, Institutes for Information Processing, Graz University of Technology

[Zac] Zachar, Z., The solvability of the equivalence problem for deterministic frontier-to-root tree transducers, Acta Cybernetica 4 (1979), 167-177. 